Old Dominion University

ODU Digital Commons

Mechanical \& Aerospace Engineering Theses \&

Dissertations

Mechanical \& Aerospace Engineering

Spring 2020

\title{
Trajectory Simulation With Battery Modeling for Electric Powered Unmanned Aerial Vehicles
}

\author{
Ege Konuk \\ Old Dominion University, egekonuk@gmail.com
}

Follow this and additional works at: https://digitalcommons.odu.edu/mae_etds

Part of the Aerospace Engineering Commons, and the Power and Energy Commons

\section{Recommended Citation}

Konuk, Ege. "Trajectory Simulation With Battery Modeling for Electric Powered Unmanned Aerial Vehicles" (2020). Master of Science (MS), Thesis, Mechanical \& Aerospace Engineering, Old Dominion University, DOI: $10.25777 / 15 b x-r e 63$

https://digitalcommons.odu.edu/mae_etds/310

This Thesis is brought to you for free and open access by the Mechanical \& Aerospace Engineering at ODU Digital Commons. It has been accepted for inclusion in Mechanical \& Aerospace Engineering Theses \& Dissertations by an authorized administrator of ODU Digital Commons. For more information, please contact digitalcommons@odu.edu. 
TRAJECTORY SIMULATION WITH BATTERY MODELING FOR ELECTRIC POWERED UNMANNED AERIAL VEHICLES

\author{
by \\ Ege Konuk \\ B.S.A.E. 2017, University of Turkish Aeronautical Association \\ A Thesis Submitted to the Faculty of \\ Old Dominion University in Partial Fulfillment of the \\ Requirements for the Degree of \\ MASTER OF SCIENCE \\ MECHANICAL AND AEROSPACE ENGINEERING \\ OLD DOMINION UNIVERSITY
}

May 2020

Approved by:

Drew Landman (Director)

Oktay Baysal (Member)

Brett Newman (Member) 


\begin{abstract}
TRAJECTORY SIMULATION WITH BATTERY MODELING FOR ELECTRIC POWERED UNMANNED AERIAL VEHICLES

Ege Konuk

Old Dominion University, 2020

Director: Dr. Drew Landman
\end{abstract}

Fixed wing electric powered unmanned aerial vehicles (UAVs) has been widely adopted for the last decade in a great number of applications. One of the primary advantages to fixed wing versus multi-rotor designs is the efficiency in forward flight with best possible range and endurance capabilities. In electrically powered air vehicles range and endurance are monitored by the State-of-Charge (SOC) of the battery. To understand the capabilities of the battery, discharge experiments can be conducted in lab environments; however, sometimes the results are difficult to integrate in flight simulations.

In this thesis, a trajectory simulation is developed that can estimate an instantaneous SOC and terminal voltage of the Lithium Polymer (Li-Po) battery of a fixed wing UAV. The simulation code is generated using the traditional flight dynamics equations for a mathematical five degree of freedom (5-DOF) system in the MATLAB environment. Simplistic control relations are defined for setting the pitch angle $(\theta)$ and roll angle $(\phi)$ of the UAV. An AVISTAR ELITE RC model has been chosen to simulate the flight mission with the goal of future flight test validations.

Initially, battery simulation was carried out in the ODU UAV lab by discharging a 3300Mah Li-Po battery to half capacity with constant current over a range of current draw. Later, these constant current discharge curves were converted to the constant power curves which are more suitable for the battery powered aircraft applications. Simulated battery pulse discharge tests 
were also conducted, and battery parameters were estimated in SIMULINK for the validation of the constant power method used in the simulation. The overall results of this research demonstrate the endurance and range of the electric UAV for mission paths that include takeoff, climb/descent and turning flight phases. 
Copyright, 2020, by Ege Konuk, All Rights Reserved. 


\section{ACKNOWLEDGMENTS}

I would like to express my huge thanks to my parents whom have always supported and giving me moral support through the toughest part of my thesis and overall graduate school. Also, I would like to express my gratitude to my thesis advisor, Professor Drew Landman, for giving all the guidance and patience throughout the period of this thesis being prepared.

Furthermore, I want to thank my dear friend Ömür İcke to all his motivational effort to help me get on track for the challenging portions of my studies. 
NOMENCLATURE

\begin{tabular}{|c|c|c|c|}
\hline$U A V$ & Unmanned Air Vehicle & $q_{\infty}$ & Dynamic pressure \\
\hline$U A S$ & Unmanned Air Systems & $\eta_{m}$ & Motor efficiency \\
\hline DOF & Degrees of Freedom & $\eta_{p}$ & Propeller efficiency \\
\hline$R C$ & Radio Controlled & $C_{T}$ & Thrust coefficient \\
\hline$\beta$ & Heading Angle & $C_{P}$ & Power coefficient \\
\hline$\phi, \mu$ & Bank Angle & $\phi_{d}$ & Desired bank angle \\
\hline$\delta$ & Control surface deflection & $n$ & Battery collapsed curve \\
\hline$\gamma$ & Flight Path Angle & coefficient & \\
\hline$V$ & Flight Velocity & $h$ & Simulation step size \\
\hline$\psi$ & Yaw Angle & $e$ & Runge-Kutta error term \\
\hline$\theta$ & Pitch Angle & $\Theta$ & RLS coefficient \\
\hline$\alpha$ & Angle of attack & $\xi$ & Runway friction Coefficient \\
\hline EOM & Equations of Motion & $\Phi$ & RLS coefficient \\
\hline$B M S$ & Battery Management Systems & $\tau_{1}$ & Heading control parameter \\
\hline$E C M$ & Equivalent Circuit Modelling & $T$ & Thrust \\
\hline$O C V$ & Open Circuit Voltage & C & Battery capacity \\
\hline$L i-P o$ & Lithium Polymer & $I$ & Current \\
\hline$R L S$ & Recursive Least Squares & $W$ & Weight \\
\hline$R$ & Battery internal resistance & $C D$ & Drag coefficient \\
\hline$C$ & Capacitance of the battery & $C L$ & Lift coefficient \\
\hline Em & Open circuit voltage & $S_{G}$ & Take-off distance \\
\hline
\end{tabular}




\section{TABLE OF CONTENTS}

Page

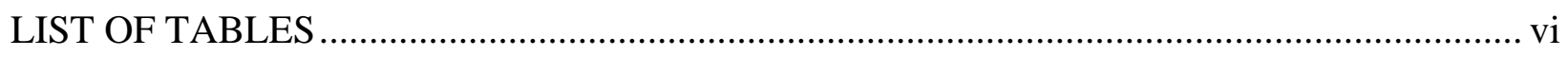

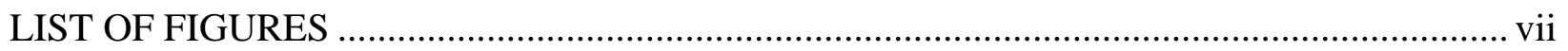

Chapter

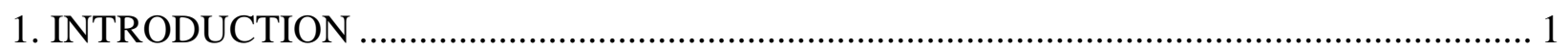

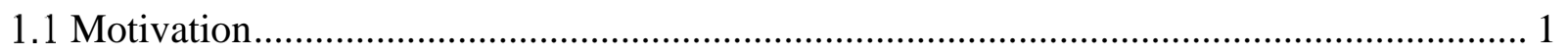

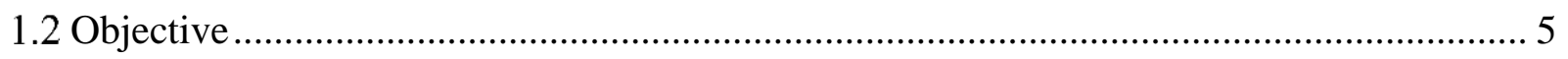

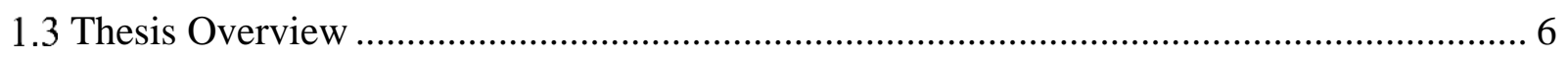

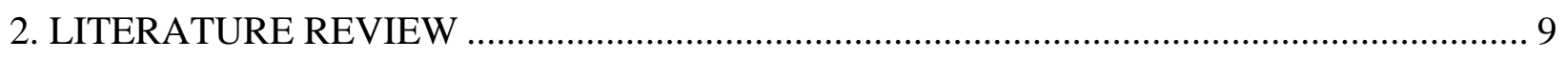

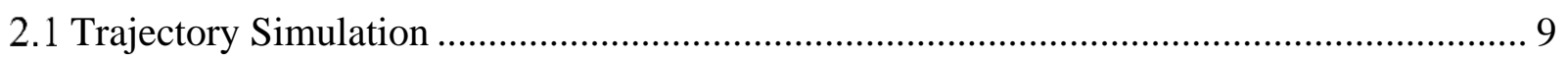

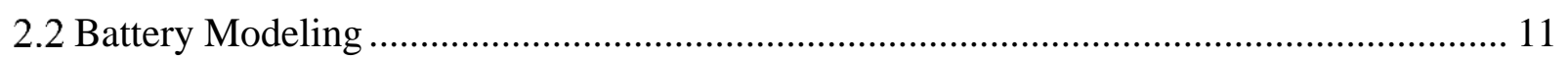

2.2.1 Coulomb Counting Method ............................................................................... 11

2.2.2 Voltage Method ................................................................................................ 13

2.2.3 Model Based Battery Performance Estimation ....................................................... 14

2.2.4 Parameter Estimation ....................................................................................... 16

2.2.5 Recursive Least Squares ............................................................................... 17

2.2.6 Parameter Estimation Using SIMULINK/MATLAB .......................................... 18

2.2.7 Constant Power Battery Curves Using Coulomb Method ...................................... 22

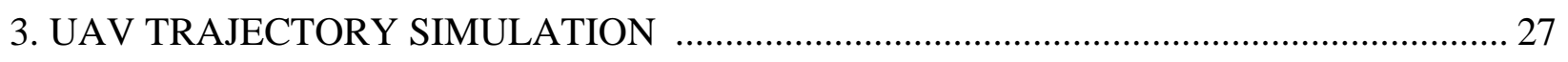

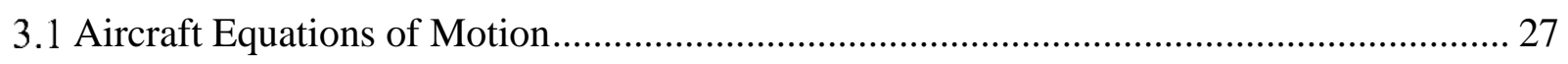

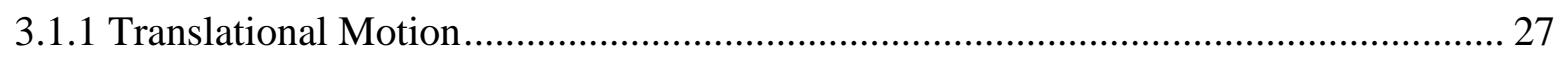

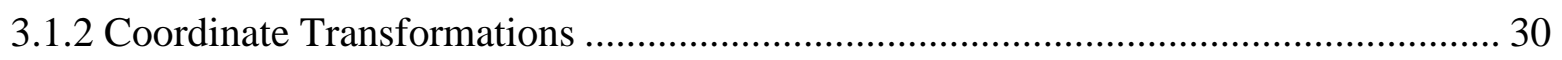

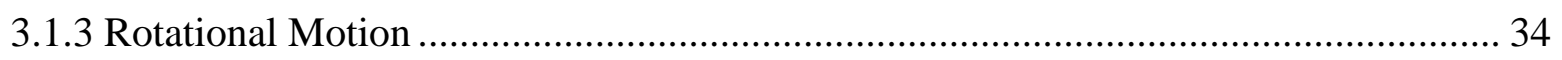

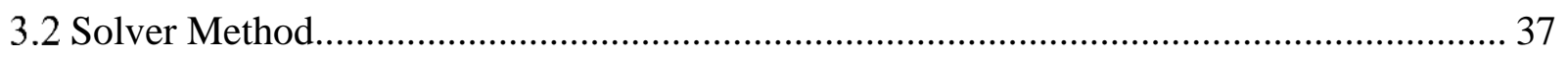

3.2.1 Runge-Kutta-Fehlberg Method with Adaptive Step-Size .................................... 37

3.3 Basic Flight Guidance and Control........................................................................... 41

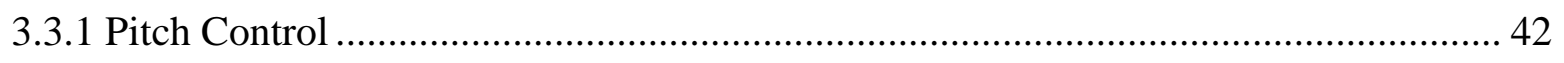

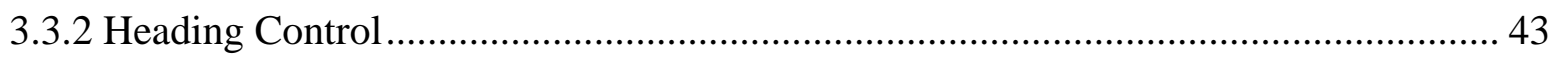

3.4 Aircraft Propulsion and Aerodynamics ................................................................. 44 
Chapter $\quad$ Page

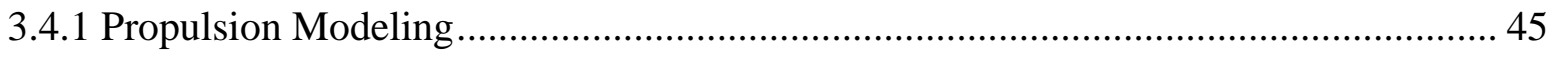

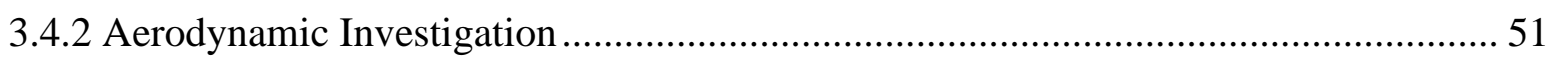

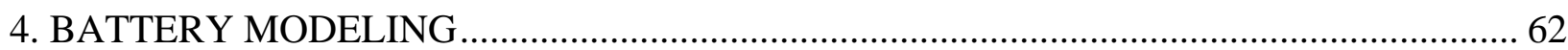

4.1 Constant Power Discharge Development ................................................................ 62

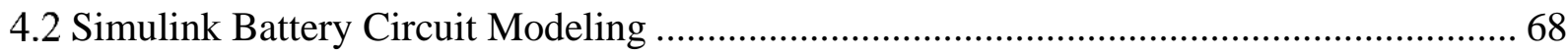

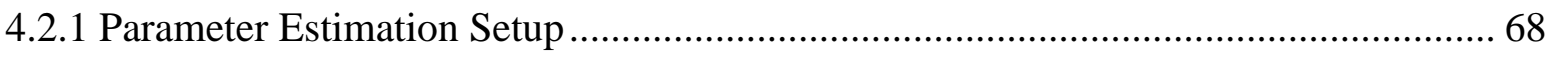

5. ELECTRIC UAV SIMULATION OVERVIEW ............................................................. 80

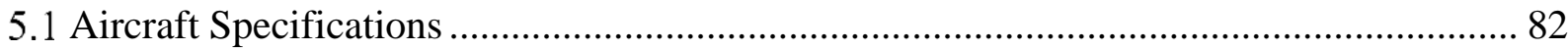

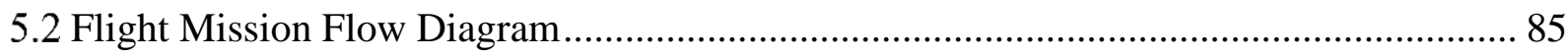

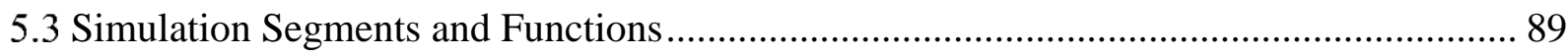

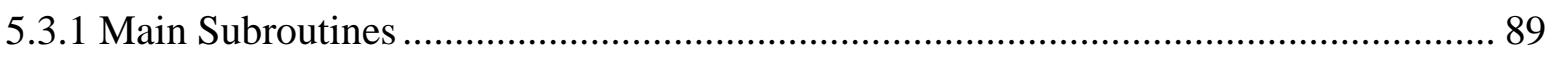

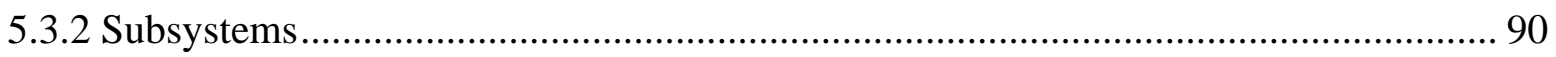

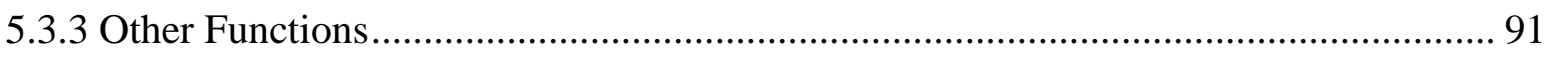

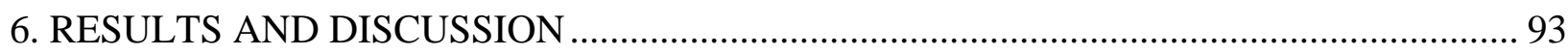

6.1 Cruise Height Benchmark Analysis........................................................................ 93

6.2 Turning Mission Benchmark Analysis ................................................................... 108

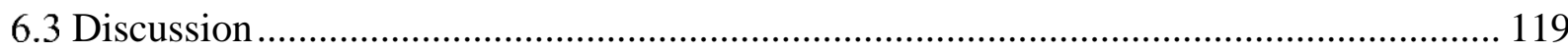

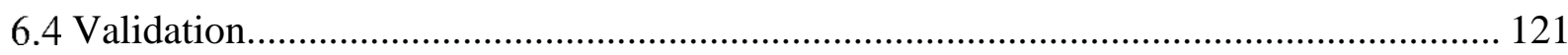

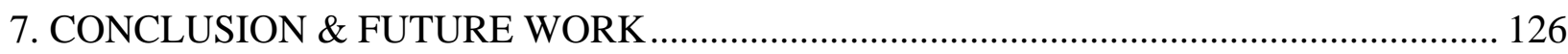

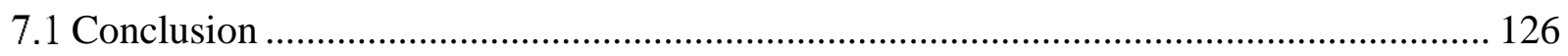

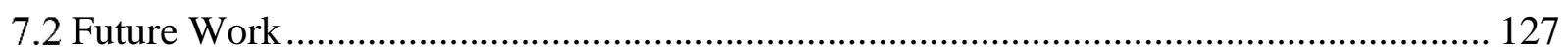

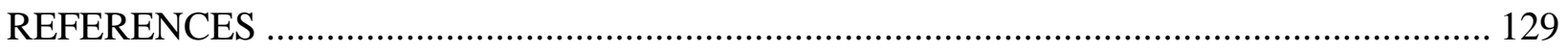

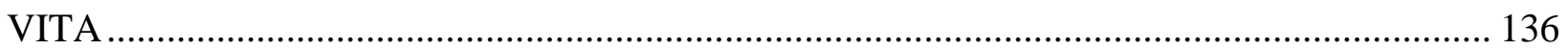




\section{LIST OF TABLES}

Table Page

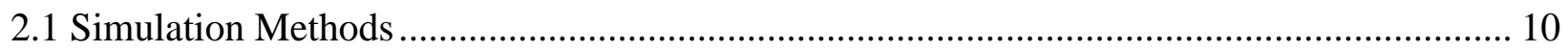

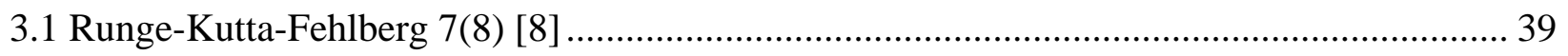

3.2 Rimfire .46 Brushless Motor Specifications ............................................................ 46

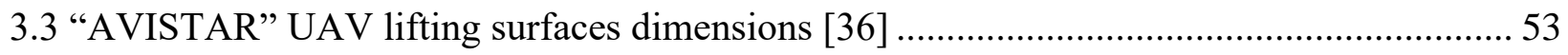

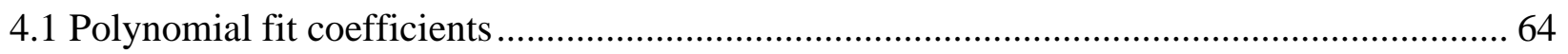

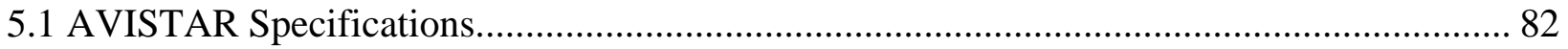

6.1 Range values for each set of simulation ................................................................. 96

6.2 Take-off run calculation parameter for ranging RPM values from the simulation.............. 121

6.3 Refined final parameters for Take-off ................................................................... 122

6.4 flight data and simulation velocity comparison with various RPM levels ........................ 124 


\section{LIST OF FIGURES}

Figure

Page

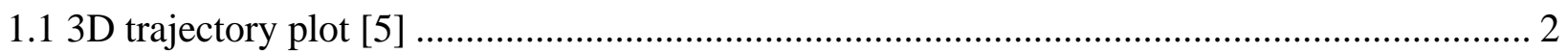

1.2 Avistar Elite Battery powered RC aircraft [6] ......................................................... 3

2.1 Typical OCV-SOC graph for lithium-based batteries [15] ........................................... 12

2.2 Difference in error term between corrected coulomb method and classic coulomb method.. 13

2.3 The commonly used battery equivalent circuit models (ECM) [17] ................................ 14

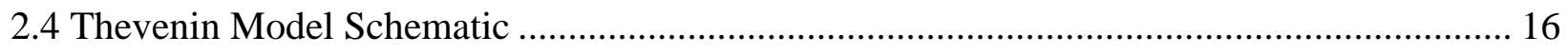

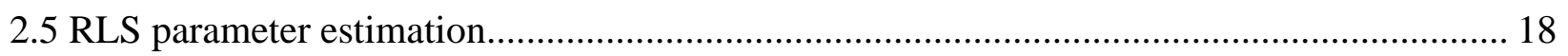

2.6 Battery Pulse Discharge experiment .......................................................................... 19

2.7 SIMULINK ${ }^{\mathrm{TM}}$ parameter estimation solution.............................................................. 21

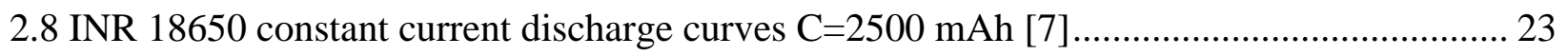

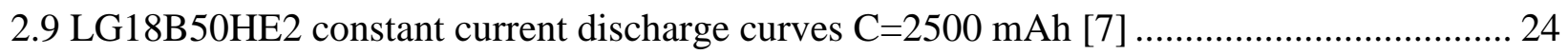

2.10 INR 18650 correlated current discharge curves. $n=0.05$ and $\mathrm{C}=2500 \mathrm{mAh}$ [7] ............ 25

2.11 LG18B50HE2 correlated current discharge curves. $\mathrm{n}=0.05$ and $\mathrm{C}=2500 \mathrm{mAh}[7] \ldots \ldots \ldots .26$

3.1 Coordinated systems over Flat Earth model ........................................................... 30

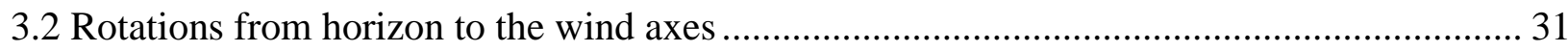

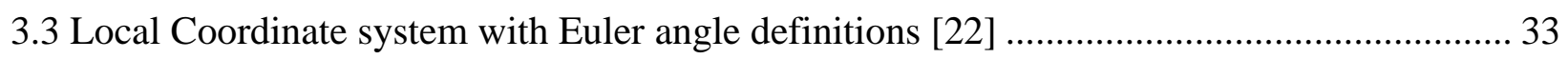

3.4 Balanced take-off free-body diagram for tricycle landing gear configuration [25].............. 37

3.5 High-level diagram of the propulsion model ............................................................ 46

3.6 Great Planes Rimfire .46-60-800 Outrunner Brushless Motor .......................................... 47

3.7 CT vs J graph for APC $12 x 6 E$ propeller RPM ranging 1000 to 17000 ............................ 49 
Figure $\quad$ Page

3.8 CP vs J graph for $A P C 12 x 6 E$ propeller RPM ranging 1000 to 17000 ............................. 50

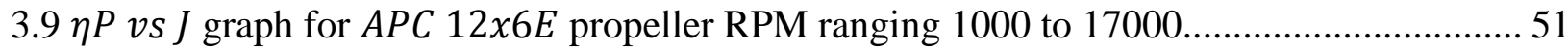

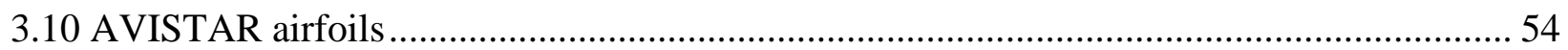

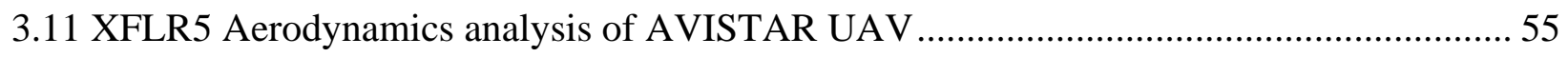

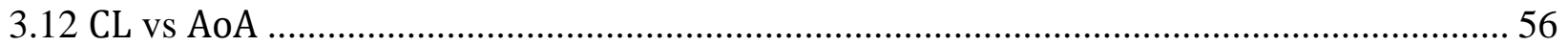

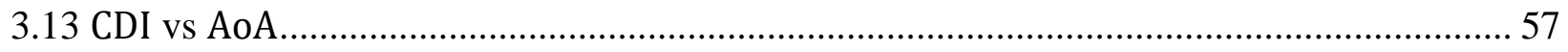

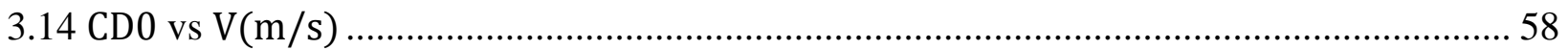

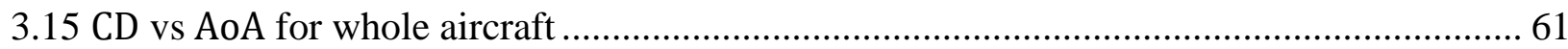

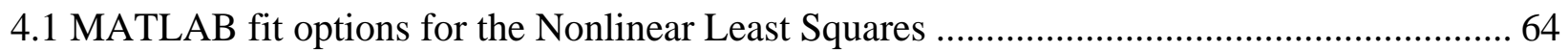

4.2 Lab battery discharge test data for PULSE 3300mAh 6S 22.2V 45C LiPo Battery ............. 66

4.3 Collapsed discharge curves $(n=0.0392)$ for PULSE LiPo Battery ................................. 67

4.4 Simulink ${ }^{\mathrm{TM}}$ library Model for single RC circuit battery modeling system........................ 69

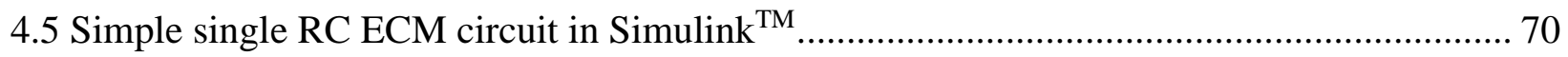

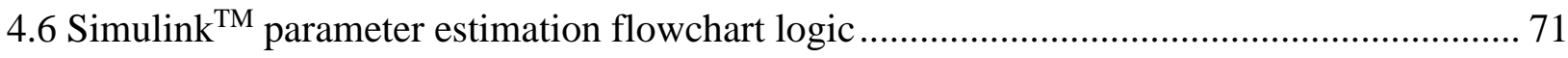

4.7 a) PULSE 3300mAh LiPo Battery b) ETEKCITY MSR-U1000 \& UNI-T UT210E ............ 72

4.8 AVISTAR RC plane fixed on the lab table for current discharge experiment ................... 72

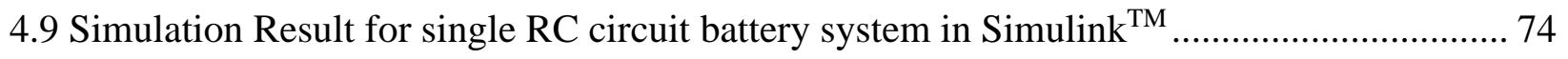

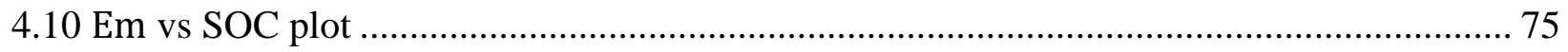

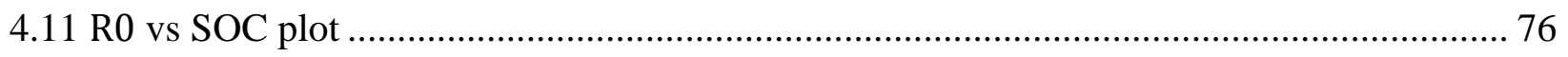

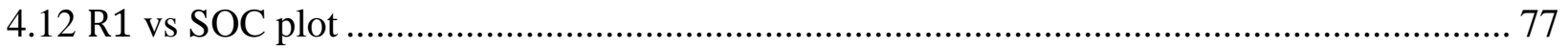

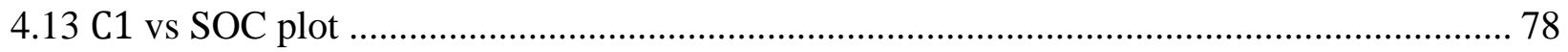


Figure

Page

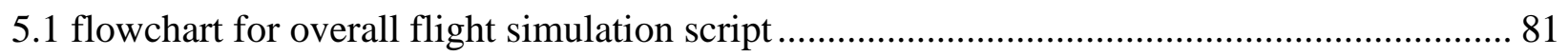

5.2 a) Phoenix Edge Lite 100 Amp ESC b) Pulse 3300 mAh 45C 6S Li-Po Battery.................. 83

5.3 Rimfire .46 Brushless Motor dimensions [49] ............................................................ 83

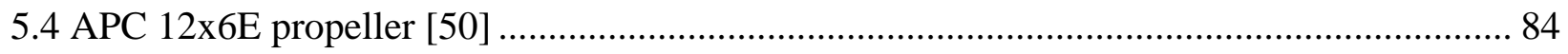

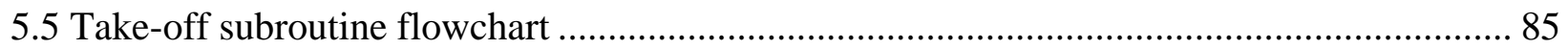

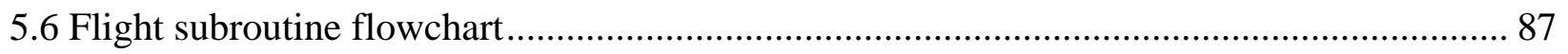

6.1 Trajectory of the Straight flight mission for $100 \mathrm{~m}$ and $200 \mathrm{~m}$ cruising altitudes................. 95

6.2 Flight attitude angles for simulation RPM = 8000 for $100 \mathrm{~m}$ cruising altitude................... 96

6.3 Flight attitude angles for simulation RPM = 9000 for $100 \mathrm{~m}$ cruising altitude .................... 97

6.4 Flight attitude angles for simulation RPM = 10000 for $100 \mathrm{~m}$ cruising altitude ..................97

6.5 Flight attitude angles for simulation RPM = 11000 for $100 \mathrm{~m}$ cruising altitude..................98

6.6 Flight attitude angles for simulation RPM = 8000 for $200 \mathrm{~m}$ cruising altitude ....................98

6.7 Flight attitude angles for simulation RPM = 9000 for $200 \mathrm{~m}$ cruising altitude................... 99

6.8 Flight attitude angles for simulation RPM = 10000 for $200 \mathrm{~m}$ cruising altitude .................. 99

6.9 Flight attitude angles for simulation RPM = 11000 for $200 \mathrm{~m}$ cruising altitude ................. 100

6.10 SOC vs Time for four RPM settings in 100m or 200m cruising altitudes...................... 101

6.11 SOC Error, Traub's constant discharge method vs. SIMULINK model ......................... 101

6.12 Current drawn vs time plot for $100 \mathrm{~m}$ cruising altitude............................................... 102

6.13 Current drawn vs time plot for $200 \mathrm{~m}$ cruising altitude............................................. 103

6.14 Voltage vs Time for four RPM settings at 100m altitude.......................................... 104

6.15 Voltage vs Time for four RPM settings at 200m altitude ......................................... 104 
Figure $\quad$ Page

6.16 Velocity vs Time for four RPM settings in 100m or 200m cruising altitudes.................. 105

6.17 Thrust vs Time for four RPM settings in 100m or 200m cruising altitudes .................... 106

6.18 Power vs Time for four RPM settings in 100m ................................................... 107

6.19 Efficiencies of the drivetrain components with time at 100m cruising altitude ............... 107

6.20 Computation time for all simulations ................................................................... 108

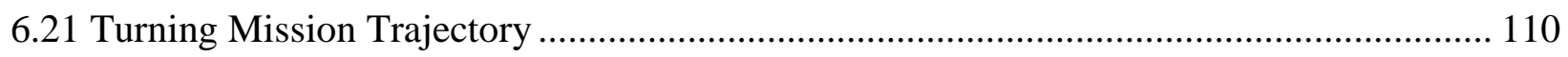

6.22 Velocity Profile of the Turning missions ranging RPM values ...................................... 111

6.23 Bank angle vs Time for each RPM values ............................................................. 111

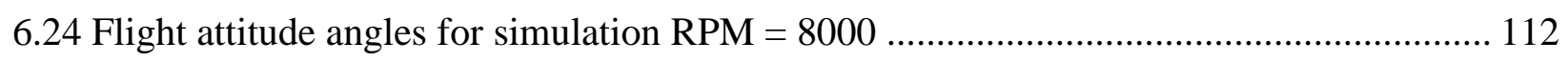

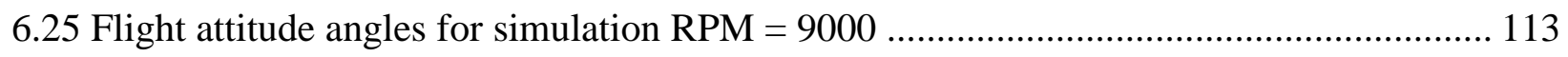

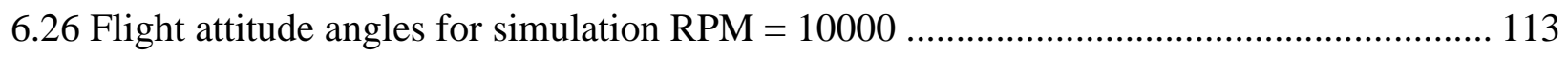

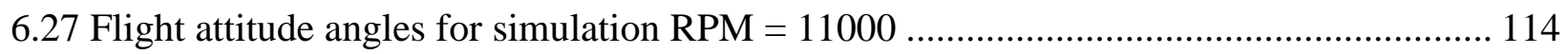

6.28 SOC vs Time plot with ranging RPM values........................................................ 115

6.29 Current vs Time plot for ranging RPM values with SIMULINK simulation points ......... 115

6.30 Voltage drop plot over simulation time for ranging RPM values................................ 116

6.31 Voltage drop plot for RPM = 8000 compared with the SIMULINK values ................... 117

6.32 Voltage drop plot for RPM = 9000 compared with the SIMULINK values .................... 117

6.33 Voltage drop plot for RPM = 10000 compared with the SIMULINK values .................. 118

6.34 Voltage drop plot for RPM = 11000 compared with the SIMULINK values .................. 118

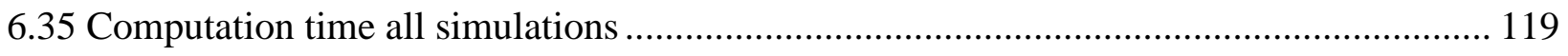

6.36 RPM vs the flight sample time from flight data ................................................. 123 
Figure

Page

6.37 Velocity vs the flight sample time from flight data .................................................. 124 


\section{CHAPTER 1}

\section{INTRODUCTION}

\subsection{MOTIVATION}

For over two decades electric powered Unmanned Air Vehicles (UAVs) have maintained their popularity in every aspect of aviation, whether civil or commercial uses, or military applications. From the day the first UAV was flown until the $21^{\text {st }}$ century, technological development of UAS (Unmanned Air Systems) has steadily increased and served many different useful applications for humanity.

Similarly, in the past decade, UAV research and development has seen a substantial increase in various commercial applications such as product/supply delivery, agricultural applications and aerial surveillance. It is known that UAV solutions rather than manned air vehicles are inherently lower in operation cost. They often go through rigorous validation and flight-testing in order to prove their capabilities in various applications, particularly those related to the military. However, conducting testing for validation of performance is not always an economically viable and timely solution. In the initial states of the aircraft design, the necessity for reliable and sufficiently accurate simulation programs has become an increasingly important factor for analyzing the performance characteristics of UAVs.

The smaller sizes of UAVs are widely utilized with different missions and objectives for various applications. When it comes to the smaller electric powered UAVs, endurance and range calculations are an issue. There have been several efforts to calculate range endurance with comparisons of design variables for different configurations of an aircraft and flight path followed 
by a UAV [1, 2, 3, 4], but these are strictly based on analytical relations and point calculation methods without utilizing the whole trajectory of the UAV. However, the high-fidelity simulation models are hard to implement and computationally intensive. A compromised method between high-fidelity simulation models and point methods is desirable. A reduced fidelity aircraft dynamics model can become useful and exploiting the approach, the optimum trajectory and control variables can be obtained for various missions.

Trajectory simulations play a significant role in calculating the performance data of any type of vehicle. Ever since the technological advancements in relating computer calculation in the 1970s, trajectory simulations have become the industry standard for determining the performance characteristics of an aircraft before performing flight testing in real-life conditions. These computer simulations help engineers make decisions and design parameters, and they provide an opportunity to achieve more efficient vehicles.

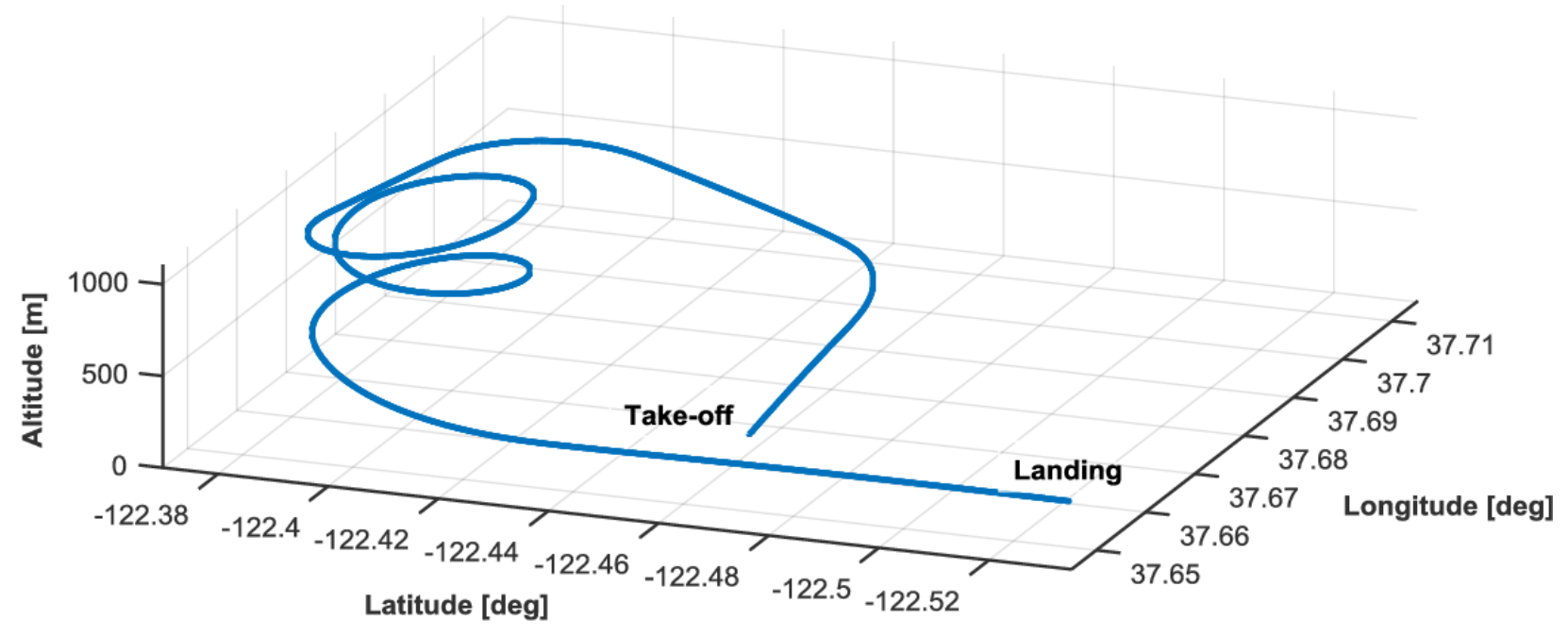

Figure 1.1 3D trajectory plot [5] 
The basis for simulating a vehicle in three-dimensional space relies on the mathematical representation of the motion that is produced under the forces that act upon the vehicle. In most cases these mathematical formulations are constructed given the assumption of a rigid body. Using RC aircraft provides a great preliminary platform for analyzing the trajectory of a UAV. Because of the simplicity of the design and operation of the aircraft, different types of tests can be conducted in a lab environment, or real-life flight tests can easily be conducted.

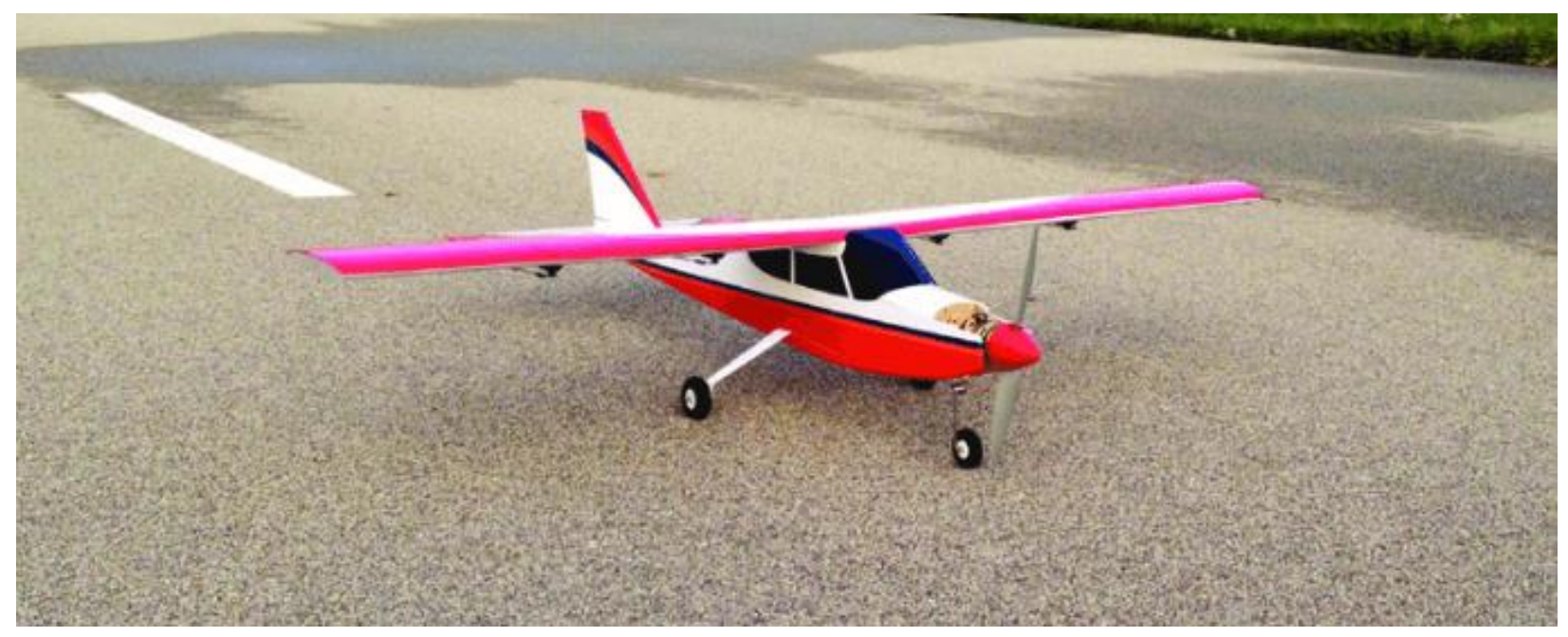

Figure 1.2 Avistar Elite Battery powered RC aircraft [6]

Another big factor for determining the performance of an electric UAV is the propulsion and energy consumption of the vehicle. Electric battery powered UAV systems have seen a significant growth in application over the last couple of decades. In most preliminary and conceptual design periods of any type of air vehicle, the estimation of the power system is the heart of the important 
selection phase for designers. Hence, engineers developed various methods to analyze the power system including more popular systems as used in the fossil fuel powered aircraft [7]. However, there are significant shortcomings in advancements between battery systems and the fossil fuel powered energy generation systems. These battery systems are devices that convert the chemical reaction energy contained in its active materials directly into electric energy by means of an electro chemical redox reaction. Battery systems generate energy by moving electrons from the anode which is the negative terminal to the cathode which is the positive terminal of the battery cell. In this chemical compound that is specific to metals or oxides used, the terminal portions of each battery cell undergo an electrochemical reaction. In most rechargeable systems, the battery is recharged by reversing the same process.

Electric propulsion has many advantages over combustion powered air vehicles. The most pronounced advantages are; zero emissions, reduced noise and weight, and responsive control compared to the combustion type engines that rely on fossil fuels [8]. Batteries fall under two main categories [9].

Those with the purpose of powering portable electronic and electric devices, with slow current draw such as cell phones, are produced to last only for one charge-discharge cycle. They have no possible use in propulsive purposes to create enough thrust to propel an air vehicle. Clearly, they are not very feasible to be used as an energy source for a UAV [9]. The second category belongs to batteries that can be recharged electrically and after recharge they maintain their original condition under the right circumstances. They are called "storage batteries" because they can provide high current flow on load demand. Also, these batteries can provide considerably higher discharge rates(C-rate) than the low-current draw category and lend themselves to 
application in electric vehicles. The discharging is essentially done the same as the primary battery, but they are capable of recharge after use which is beyond the capabilities of the primary batteries. Examples of these batteries are mainly lead-acid, nickel-cadmium (NiCd), nickel-metal hydride (NiMH), lithium-ion (Li-ion), and lithium-ion polymer.

There is also one more major electrical power source known as "Fuel cells". Fuel cells are the electrochemical galvanic cells that convert chemical energy directly into electrical energy, meaning that there are no Carnot cycle limitations which exist on combustion engines. Fuel Cells differ from the other two types mainly because the energy production is only possible if the active materials are fed to the electrodes which means this type of battery will cease to produce electrical energy when the reactant material is consumed totally. Hence, in the interest of this thesis, consideration of the electrical source is limited to the secondary type, high discharge batteries.

\subsection{OBJECTIVE}

This thesis is demonstrating a dynamic solution for a flight mechanics model in three-dimensional space with a mathematical five degree of freedom(5-DOF) model. The UAV used in the simulation is available as a physical RC model, yielding a possibility to perform experimental tests with the RC plane, collect data, and compare to the main method of this thesis. The method generated in this thesis follows trajectory analysis methods but with the addition of a new propulsive and power subsystem component. There is a separate comprehensive investigation carried out with modeling and simulating the electrical propulsion of this category of air vehicle. The validation for the battery and power calculation models is made with a commercial tool available that has established methods for similar simulations. Hence, the overall trajectory of the determined mission profile will be flown using the dynamics model created in the MATLAB environment with simple 
guidance and control logic to successfully fly the same mission profile. The final objective is to show and compare the different mission profiles and investigate the difference in SOC and the terminal voltage left in the battery for each flight mission being flown.

\subsection{THESIS OVERVIEW}

The work presented in this thesis is divided into six chapters. The first chapter gives a description of the overall objective of this simulation effort and reasons behind the platform and the methods that are being chosen in this thesis.

The second chapter describes the background theory of dynamics and battery modelling that are being analyzed in the later chapters, while giving the definitions of other possible options for the battery and dynamics simulation and the overall advantages of the method used in the thesis. Also, it deals with the parameter estimation process for the lithium-based batteries that are used in the battery modelling while demonstrating the lab experiment that is conducted for physical representation of the power calculation. Finally, it introduces a constant power discharging approach which simplifies the modelling procedure.

Chapter 3 showcases the trajectory analysis where the derivation of equations of motion are performed to define the dynamics model for the simulation space. The solver method is also defined in this chapter with an adaptive time-step feature being used. Later in the chapter basic flight guidance and control methods are described and the integration to the model is defined by calculating the simple control parameters. The last section in this chapter is dedicated to the subsystems which are propulsion and aerodynamics.

In chapter 4 detailed analysis is carried out for the battery modelling portion of the simulation. This chapter gives a step by step method for generating a constant power discharge 
approach to battery modeling and demonstration of the experimental values and estimated values being shown. The experimental setup is defined in this chapter with the SIMULINK model that is constructed for validation purposes. The parameter estimation coefficients are then calculated at the end of this chapter.

In chapter 5 a detailed specification for the AVISTAR UAV is provided, and the flight mission flow diagram is also explained with the flight phases that are defined for this mission. Lastly, input and output for the simulation is defined at the end of this chapter.

Finally, Chapter 6 presents the simulation results of the electric powered UAV in the MATLAB environment. Recommendations and future work are defined. 


\section{CHAPTER 2}

\section{LITERATURE SEARCH}

\subsection{TRAJECTORY SIMULATION}

The equations of motion are composed of translational-force and rotational-moment equations. Simulations are developed depending on the required fidelity, and the involved equations of motion are commonly divided by the number of degrees of freedom they concern about the body in motion. These equations define the motion of aircraft (3-D object in space) by using the inertia and the angular acceleration of the vehicle and treating the vehicle as a 3-D rigid body. The methods described in this section treat the vehicle as a point in space; hence, the dynamic equations are called "Mathematical DOF" [10]. By this definition, the number of mathematical degrees of freedom of a differential system is equal to the difference between the combination of the state variables and the control (inputs) variables from the Number of Equations (EOMs). Commonly used methods for completely simulating a vehicle in a three-dimensional environment like earth's atmosphere or space are called six degree of freedom (6-DOF) equations of motion. 6-DOF simulation contains all of the motions (defined by states) that an air-vehicle can experience, and these consist of three position coordinates in three-dimensional space $(x, y, z)$ plus the pitch, yaw and roll motions. Table 2.1 summarizes the possible simulation models that are commonly used in aircraft simulations [11]. 


\begin{tabular}{|c|c|c|c|c|}
\hline Model & States & Controls & EOM & Constraints \\
\hline $\begin{array}{l}2 \text { DOF navigation }+1 \\
\text { DOF point mass }\end{array}$ & $x, y, \beta$ & $\dot{\beta}$ & $\beta$ & $\dot{\beta} \leq \frac{V}{R_{\min }}$ \\
\hline $\begin{array}{l}3 \text { DOF navigation+ } \\
1 \text { DOF point mass }\end{array}$ & $x, y, z, \beta$ & $\dot{\beta}, \gamma$ & $\dot{x}, \dot{y}, \dot{z}$ & $\gamma \leq \gamma_{\max }$ \\
\hline $\begin{array}{l}3 \text { DOF navigation }+ \\
2 \text { DOF point mass }\end{array}$ & $x, y, z, \beta, \gamma$ & $\alpha, T, \phi$ & $\dot{V}, \dot{\gamma}, \dot{\beta}$ & $\begin{aligned} T & \leq T_{\max } \\
C L & \leq C L_{\max } \\
\phi & \leq \phi_{\max }\end{aligned}$ \\
\hline $\begin{array}{l}3 \text { DOF navigation+ } \\
3 \text { DOF point mass }\end{array}$ & $x, y, z, \beta, \theta, \psi, p, q, r$ & $\alpha, T, \phi, \delta_{E, A, R}$ & $\dot{x}, \dot{y}, \dot{z}, \dot{p}, \dot{q}, \dot{r}, \dot{u}, \dot{v}, \dot{w}$ & $\begin{array}{c}T \leq T_{\max } \\
C L \leq C L_{\max } \\
\phi \leq \phi_{\max } \\
\delta_{E, A, R} \\
\leq \delta_{(E, A, R)_{\max }}\end{array}$ \\
\hline
\end{tabular}

Table 2.1 Simulation Methods

Each EOM method provides a set of parameters to solve the differential problem. Lesser degree of freedom solutions are very useful at simulating simplistic environments such as twodimensional trajectory calculations or solely translational motions. However, in the threedimensional environment, higher degrees of freedom are required to capture all aspects of flight. Vertical and horizontal flight equations derived from the scalar equations of motion are coupled in the higher order systems.

As for the goal of this thesis, interest is in managing a full mission path including climb and descent as well as turning flight. The 6-DOF method body forces are calculated with moments at the center of gravity of the aircraft. The solution of the force and moment equilibrium tensors are calculated in the aircraft body-axis system (moving-axis). Motion is then transformed to the inertial coordinate system (fixed axis) using Euler angles $(\phi . \psi, \theta)$ or quaternions $(i, j, k)$ based 
systems. Most common techniques in aircraft simulations use Euler angles since they provide a physical meaning to the parameters in three-dimensional space [12].

A similar technique is used in 5-DOF simulations which shows a significant decrease in complexity of integration versus the 6-DOF models. The main difference between them is the yaw motion is included in the 6-DOF model as a coupled derivative with the control surfaces of the aircraft. The 5-DOF model neglects the yaw motion and always considers coordinated rolling and turn reaction. This a natural behavior in un-disturbed environments and fixed reference frames and it is a good compromise between integration and complexity for the purpose of this thesis.

\subsection{BATTERY MODELING}

\subsubsection{Coulomb Counting Method}

Coulomb counting is one of most widely adopted methods for battery SOC charge estimation. As described in the previous chapter the coulomb counting method relies on simple but intuitive analysis. It basically stands for integration of current and is a simple technique for estimating the SOC by integrating current with time. Although this method is widely adopted in the early BMS studies, it has a several drawbacks. It is based on direct measurements and it does not account for self-discharge and parasitic effects of the inherent battery characteristics [13]. Hence, some researchers studied and developed different types of enhanced coulomb counting methods to address this lack of accuracy problem that the original method lacks $[14,15]$. These measurement errors accumulate with time. 


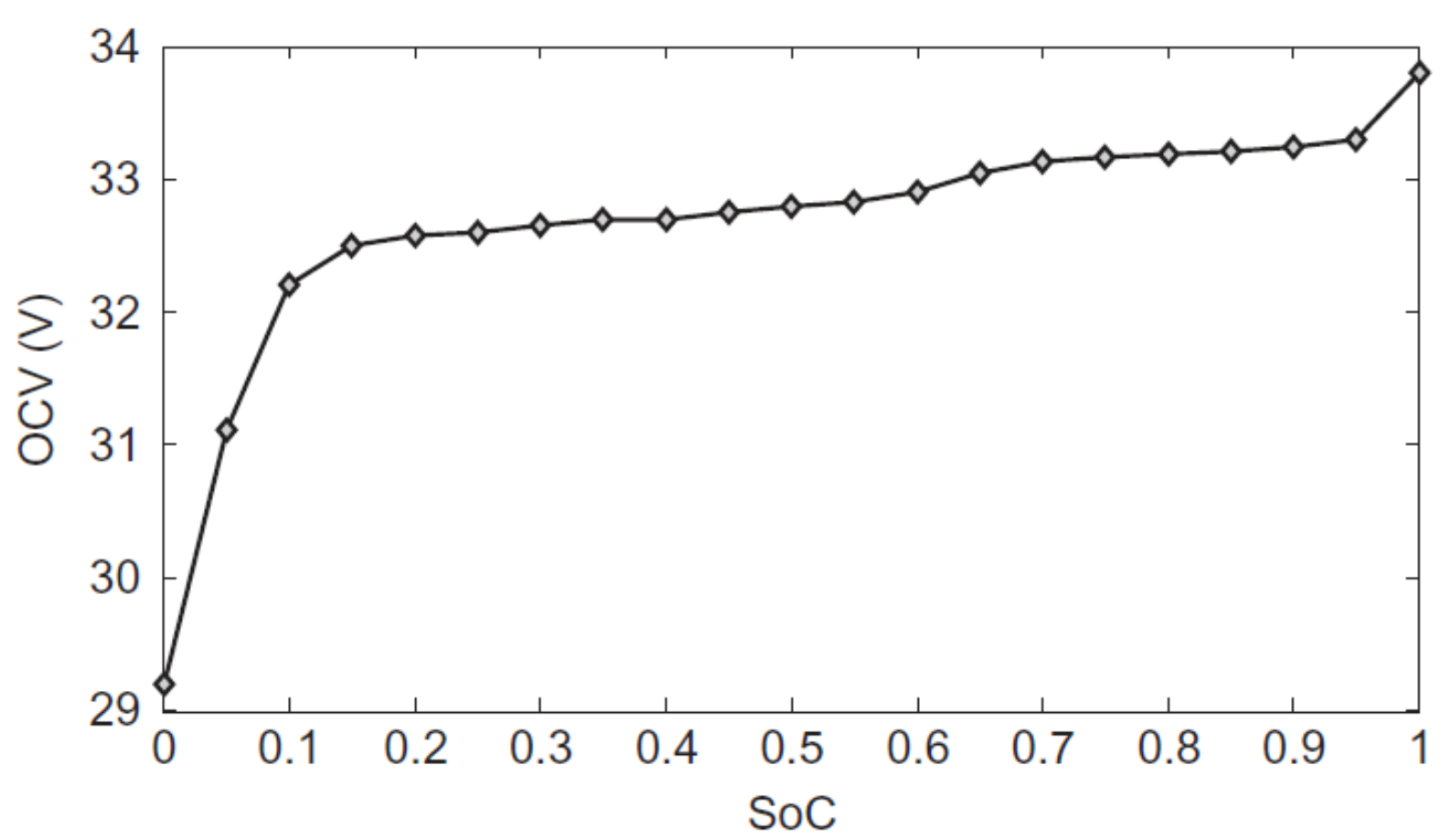

Figure 2.1 Typical OCV-SOC graph for lithium-based batteries [16]

It is still a convenient and accurate method for estimating SOC of lithium batteries [15]. This is the exact condition that is sought in this study of flight simulation of electric powered UAV. The effectiveness of the coulomb method is similar enough that the discharging efficiencies are in ranges that yield mostly accurate results for the purpose of this work. Considering that the battery used in the simulation is relatively new, according to the experiments [15] estimation errors lies in the lower percentage on and before 10 cycles of discharge. 


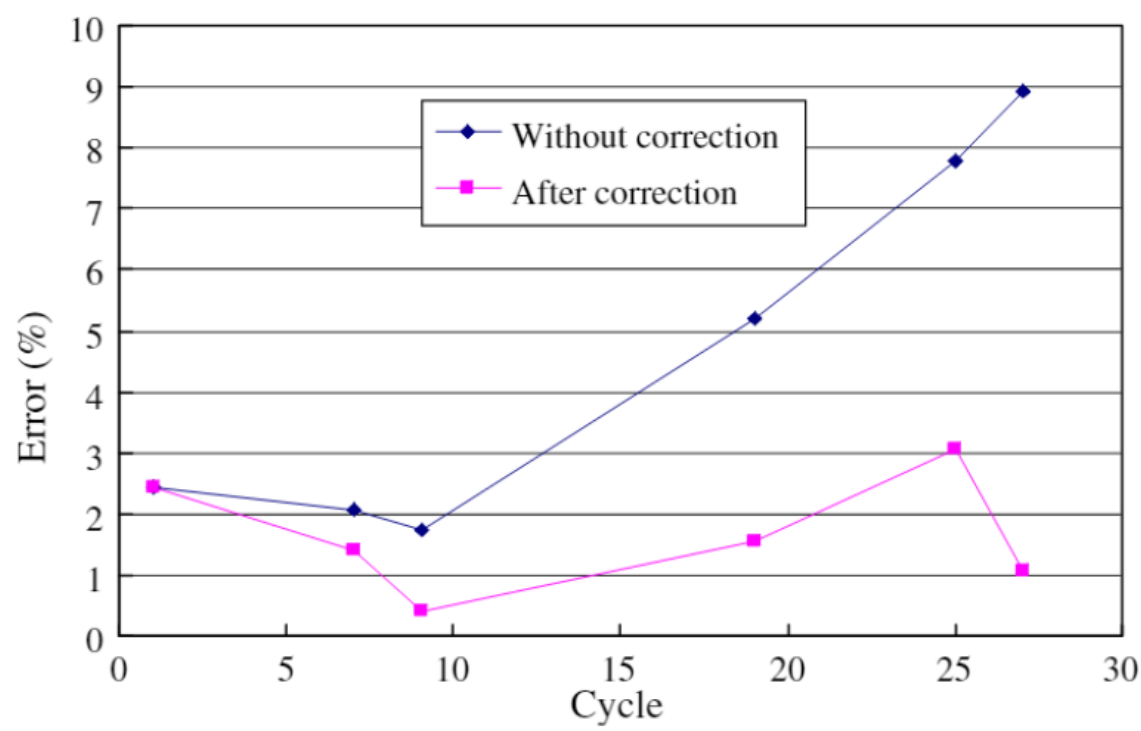

Figure 2.2 Difference in error term between corrected coulomb method and classic coulomb method with the number of experiments [15]

\subsubsection{Voltage Method}

The determination of SOC for a battery which is the remaining capacity, can be obtained using discharge test under controlled environment conditions. The voltage method converts this reading of battery voltage to the equivalent SOC value by using discharge curves. However, there is a big factor that needs to be evaluated to apply this conversion. It is known from the experimental test results that voltage drop of the battery over a discharge is majorly influenced by the current withdrawal rate of the discharge process. It is possible to make this method accurate enough to be a useful evaluation technique by compensating the voltage reading with the correction term proportional to the battery current thus using lookup table for OCV-SOC relation. There is a need 
for a stable voltage range to ensure this method's accuracy; hence, this makes this method difficult to implement in systems where the current range jumps frequently during the discharge. For that reason, the nature of this project is not suitable for this method. Another drawback is that this method is offline, so the function is interrupted during the analysis which makes it hard to apply to a dynamic system [17].

\subsubsection{Model Based Battery Performance Estimation}

Model based battery simulation methods are more sophisticated analysis techniques to estimate the SOC of the battery at a given discharge rate and the time elapsed from the previous time keeping. ECM (Equivalent Circuit Models) are the main examples of this type of battery estimation processes. ECM contains a series of resistors and one or more RC circuit blocks depending on the method.

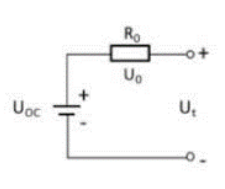

(a) Rint

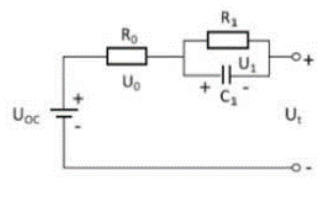

(b) Thevenin

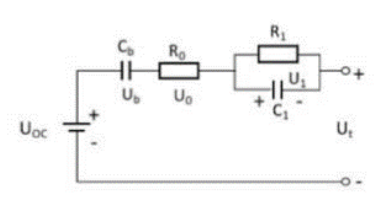

(c) PNGV

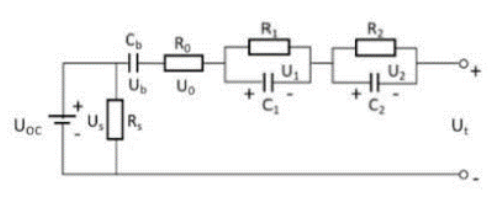

(d) GNL

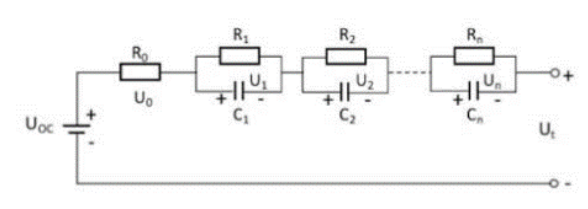

(e) n RC

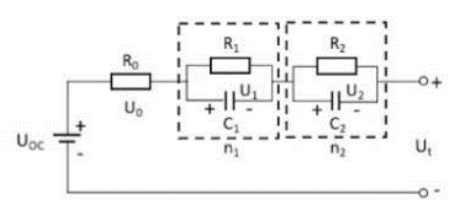

(f) FOM

Figure 2.3 The commonly used battery equivalent circuit models (ECM) [18] 
ECM is the most common approach for numerical battery estimation. The computational effort is kept relatively simple and can easily combine with other methods such as coulomb counting and voltage methods $[13,19]$. The adaptive methods such as Extended Kalman filter (EKF) can also be combined as a couple manner to provide OCV/SOC relations to the ECM methods. Although this includes the EKF (extended Kalman filter), battery performance solutions gain good precision and accuracy throughout the system. The implementation of those methods is notably hard and time consuming especially compared to book-keeping methods such as “Coulomb Counting” [14].

The main examples of this category are Rint model, Thevenin model, DP models, PNGV model, KF and EKF, Proportional integral observer, Sliding mode observer, etc. [14, 20, 18]. The Thevenin model is a good compromise between complexity and computation time; hence, this model is explained and discussed for the purpose of the simulation in the following subsection.

As defined for the Hybrid Pulse Power Characterization (HPPC) test [21], for each discharge pulse given to the battery $10 \%$ of SOC is taken from the battery. The methods suggest using a look-up table for the SOC-OCV relationship. We use the Simulink ${ }^{\mathrm{TM}}$ blocks that give a good agreement for the look-up values to estimate SOC value after each discharge period.

The initial model choice selected as the Thevenin Battery model which is popular among model-based approaches for estimating the internal battery parameters. The model contains a single RC circuit with internal(ohmic) resistance and the open circuit voltage. 


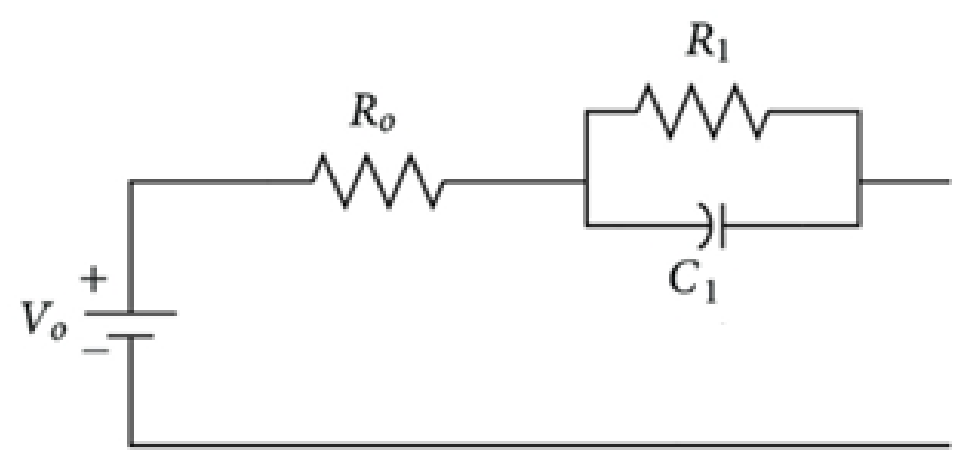

Figure 2.4 Thevenin Model Schematic

The electrical behavior is explained for the Thevenin model below.

$$
\begin{gathered}
\dot{V}_{R C}=\frac{V_{R C}}{R_{1} C_{1}}-\frac{I_{L}}{C_{1}} \\
V_{t}=E m-V_{R C}-I_{L} R_{0}
\end{gathered}
$$

$R_{0}$ is the internal resistance, $I_{L}$ is current load, $R_{1}, C_{1}$ are the corresponding resistance and capacitance of the RC circuit constructed in Thevenin model. The regression model can be created from the following differential formulation defined for the Equivalent Circuit Model for the Thevenin approach. In the formulation "Em" is used to describe the Open Circuit Voltage of the battery circuit. $V_{R C}$ is voltage across parallel RC circuit.

\subsubsection{Parameter Estimation}

As discussed in the previous section, a simple battery system consists of circuit modules. In the case of the Thevenin model there are four parameters that are needed to be known in order to calculate the voltage and corresponding SOC at a given time value. The parameter estimation 
method is commonly utilized as a mathematical optimization method which estimates the system parameters from the input and outputs of the actual system. There are a couple of different estimations. In this thesis, the "Recursive Least Squares" estimation method will be adapted to solve the Li-Po battery parameters.

\subsubsection{Recursive Least Squares}

For the name given "Recursive", parameter matrix estimation is computed recursively over time by utilizing simple modification to the covariance matrix to obtain the next current step time coefficient, as opposed to the regular least squares where the estimation output matrix is expressed with using completely previous steps coefficients [22].

Regular Least Squares:

$$
\hat{y}_{k}=\phi(k-1) \theta(k-1)
$$

Recursive Least Squares:

$$
\hat{y}_{k}=\phi(k) \theta(k-1)
$$

where $\hat{\mathrm{y}}_{\mathrm{k}}$ is the estimated system output matrix $\phi$ system excitation $\theta$ parameters matrix (covariance). The Recursive least squares estimation method works by determining the approximate parameters for the static system by minimizing the sum of the squared errors between experimental and estimated data. It is a very popular scheme mainly because it demonstrates efficient computational source consumption during continuous parameter monitoring and the online estimation process. The main algorithm for RLS is illustrated in the flow chart in Figure 2.5 . 


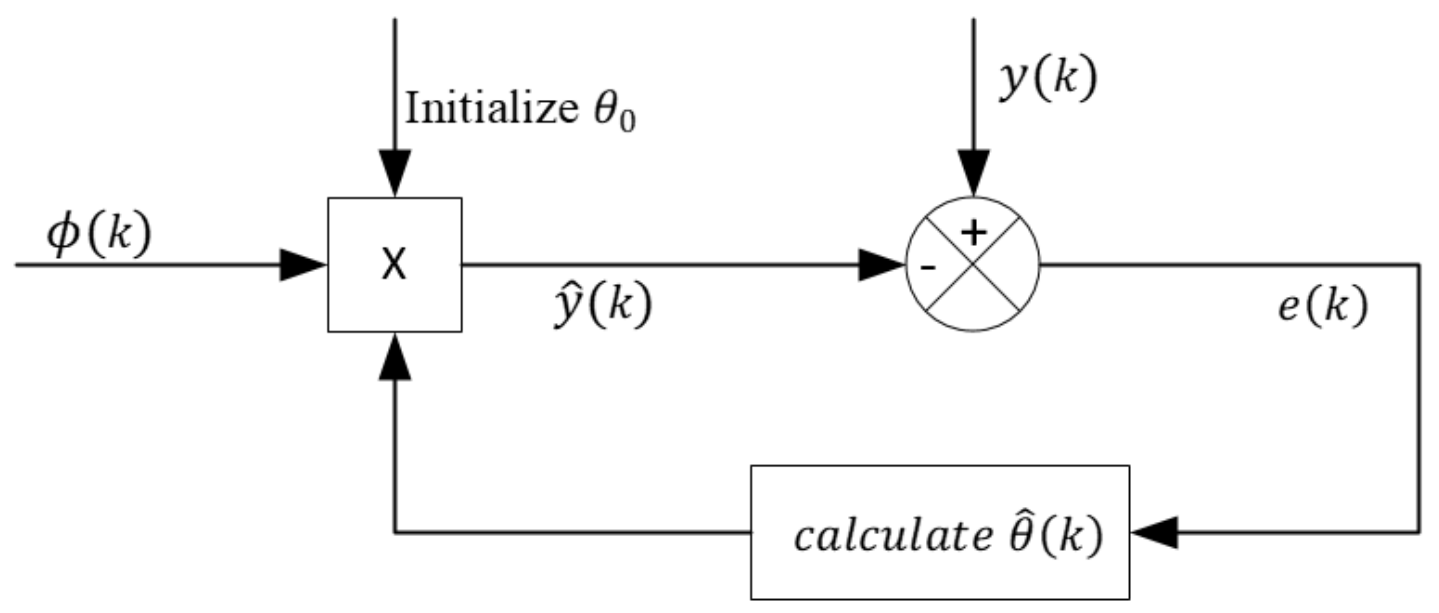

Figure 2.5 RLS parameter estimation

\subsubsection{Parameter Estimation Using SIMULINK/MATLAB}

Another way of obtaining the internal battery parameters is with the help of Simscape ${ }^{\mathrm{TM}}$ blocks using Simulink ${ }^{\mathrm{TM}}$ software. Simulink ${ }^{\mathrm{TM}}$ is capable of estimating the parameter by using MATLAB's optimization toolbox. Similar to the system developed in the previous section, the Simulink $^{\mathrm{TM}}$ parameter estimation process estimates those four parameters from the constructed ECM circuitry. In this case of analysis the ECM consists of one RC block similar to the Thevenin model explained above.

The common method of obtaining parameters using Simulink ${ }^{\mathrm{TM}}$ Parameter Estimation requires carrying out pulse discharge tests in the lab for the subject battery with constant discharge rate. There are two stages for battery analysis in the Simulink/Simscape ${ }^{\mathrm{TM}}$ environment. Initially, 
numerical analysis is performed iteratively over the experimental data at parameters estimation stage [13]. After that, results are compared with the experimental data at the validation stage. The following ECM was constructed in Simulink ${ }^{\mathrm{TM}}$ language using Simscape ${ }^{\mathrm{TM}}$ blocks. Each custom block was created to simulate the internal battery parameters. These blocks are connected to the 2D look-up tables provided by the database of Simulink ${ }^{\mathrm{TM}}$. The following experimental test is constructed at the lab:

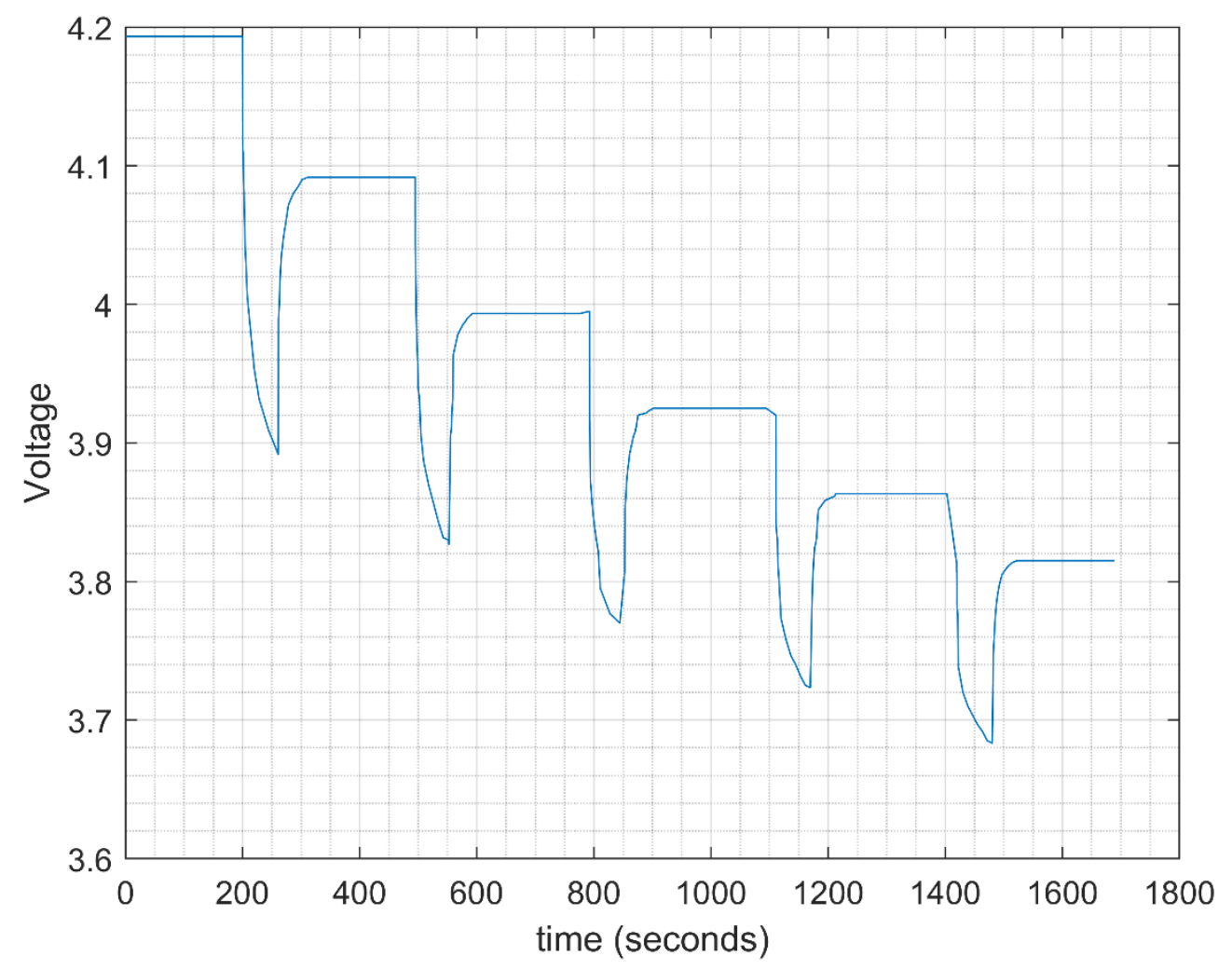

Figure 2.6 Battery Pulse Discharge experiment 
Next the ECM module was constructed to simulate one cell battery circuit in the Simulink $^{\mathrm{TM}}$ using Simscape ${ }^{\mathrm{TM}}$ blocks and language. This single RC block representation of the ECM that is used for the estimation procedure of the battery parameters was constructed using the custom electrical blocks. Simulink ${ }^{\mathrm{TM}}$ parameter estimation utilizes this circuit model for each function call during the estimation process. The estimation process is shown with an initial try for the simulation demonstrating the results plots. A similar process has been carried out for the actual estimation for the parameter for the Simulink ${ }^{\mathrm{TM}}$ model used in the simulation. 

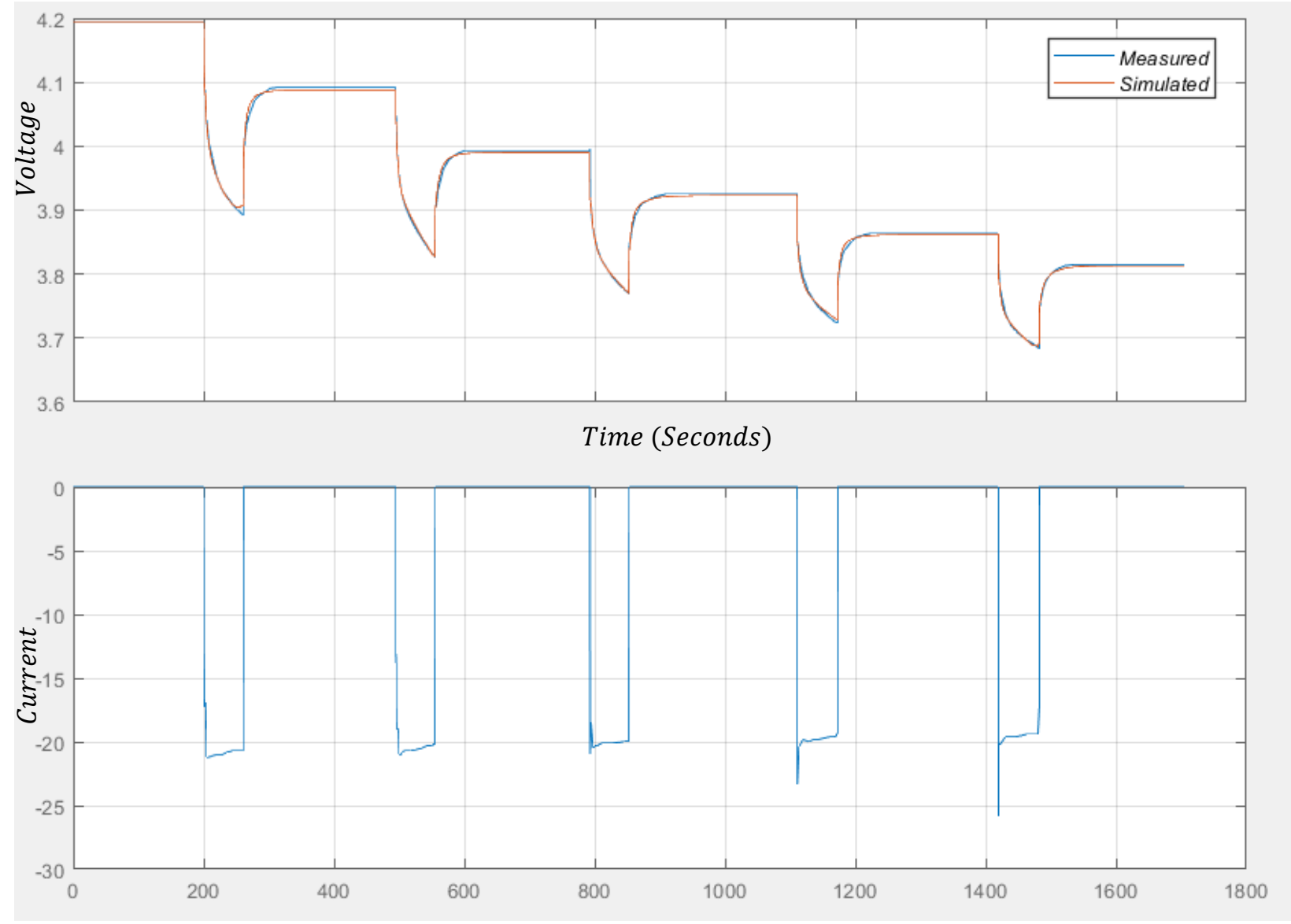

Figure 2.7 SIMULINK ${ }^{\text {TM }}$ parameter estimation solution

The parameters are attained with respect to the SOC. Note that SOC taken between $0.5-1$; this is because the experiment is only conducted at that range for a total of five pulses. In Section 4.2 the detailed solutions are presented as well as the four independent parameters with respect to the SOC.

These parameters are introduced to the Simulink ${ }^{\mathrm{TM}}$ model in flight simulation in order to calculate the battery performance and to validate the main battery model provided in this thesis. 


\subsubsection{Constant Power Battery Curves Using Coulomb Method}

Most of the model-based method utilizes a standard battery testing procedure that consists of discharging the battery at a constant current. A battery testing stand must be established in order to obtain the time varying curves relating to the given battery performance over the time by measuring the terminal voltage across the opposite poles of the battery. The capabilities of this experimental setup help us measure the OCV (open circuit voltage) with respect to the discharged capacity. However, the problem with this measurement method is that the biggest portion of the flight envelope of the UAV is a cruise stage where power is kept constant, implying that battery

character is developed over a constant power discharge. As discussed, the experimental portion of the data acquisition is performed over constant discharge or charge profiles, so standard curves would be invalid in any type of steady flight situation.

A solution for this problem is suggested by Lance W. Traub [7]. His method explains the conversion of regular constant discharge curves into flight performance accurate representation of constant power discharge solutions. Application of this technique requires the initial curves either from the manufacturer or experiments that can be conducted at constant discharges. The following figures are obtained via digitization of the manufacturer's charts according to Traub's paper [7]. 


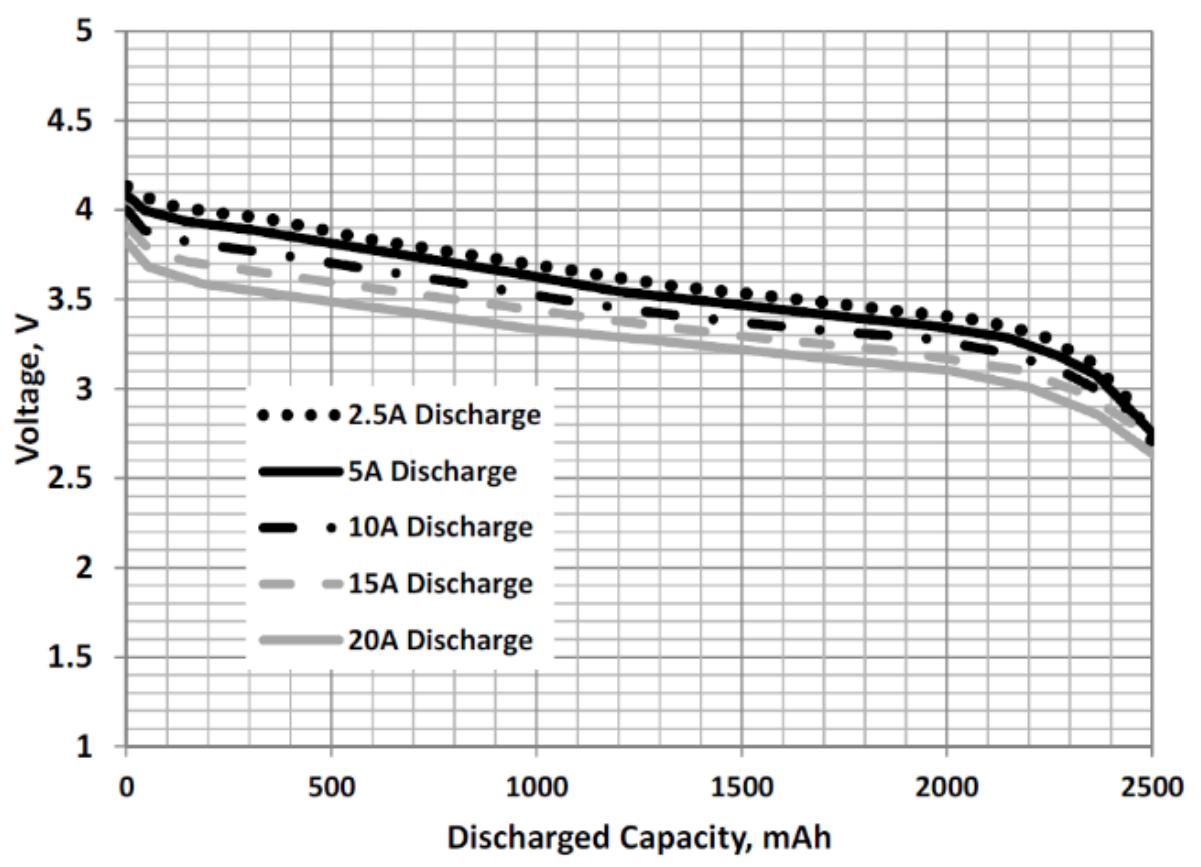

Figure 2.8 INR 18650 constant current discharge curves C=2500 $\mathrm{mAh}$ [7] 


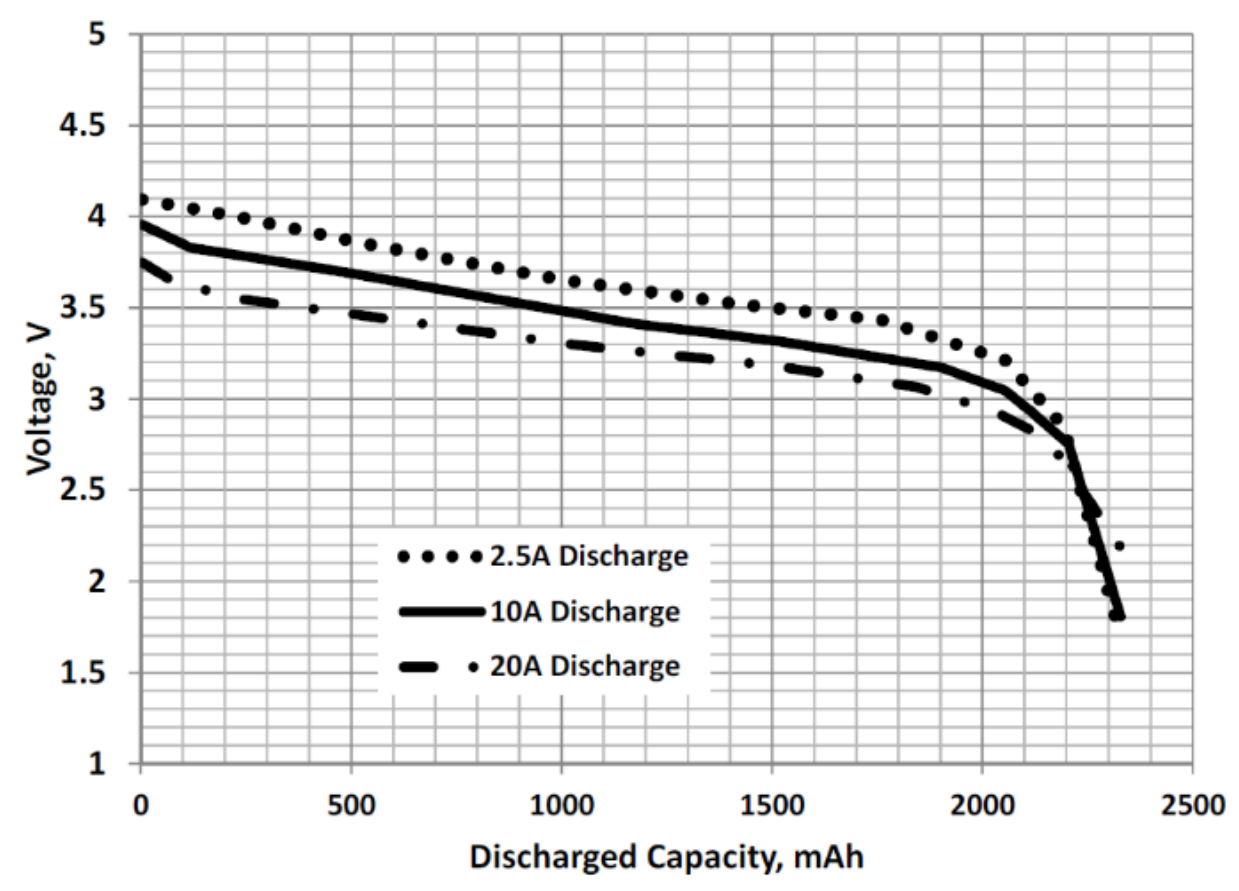

Figure 2.9 LG18B50HE2 constant current discharge curves $C=2500 \mathrm{mAh}$ [7]

As expected, higher discharge currents result in more reduction in voltage over the constant discharged capacity line. This is due to the polarization voltage increases in the electrode reaction [9]. The strategy for obtaining a uniform representation of each battery curve is to collapse the curves and try to end up with almost a single identifying discharge curve to represent different discharge rates. The collapsed curves are obtained by raising current to the power " $n$ " for a given discharged capacity then multiplying by the voltage as shown below in the corresponding collapsed curves in Figure 2.10 and Figure 2.11 obtained by this method:

$$
i^{n} V(D)=\text { Constant }(D)
$$




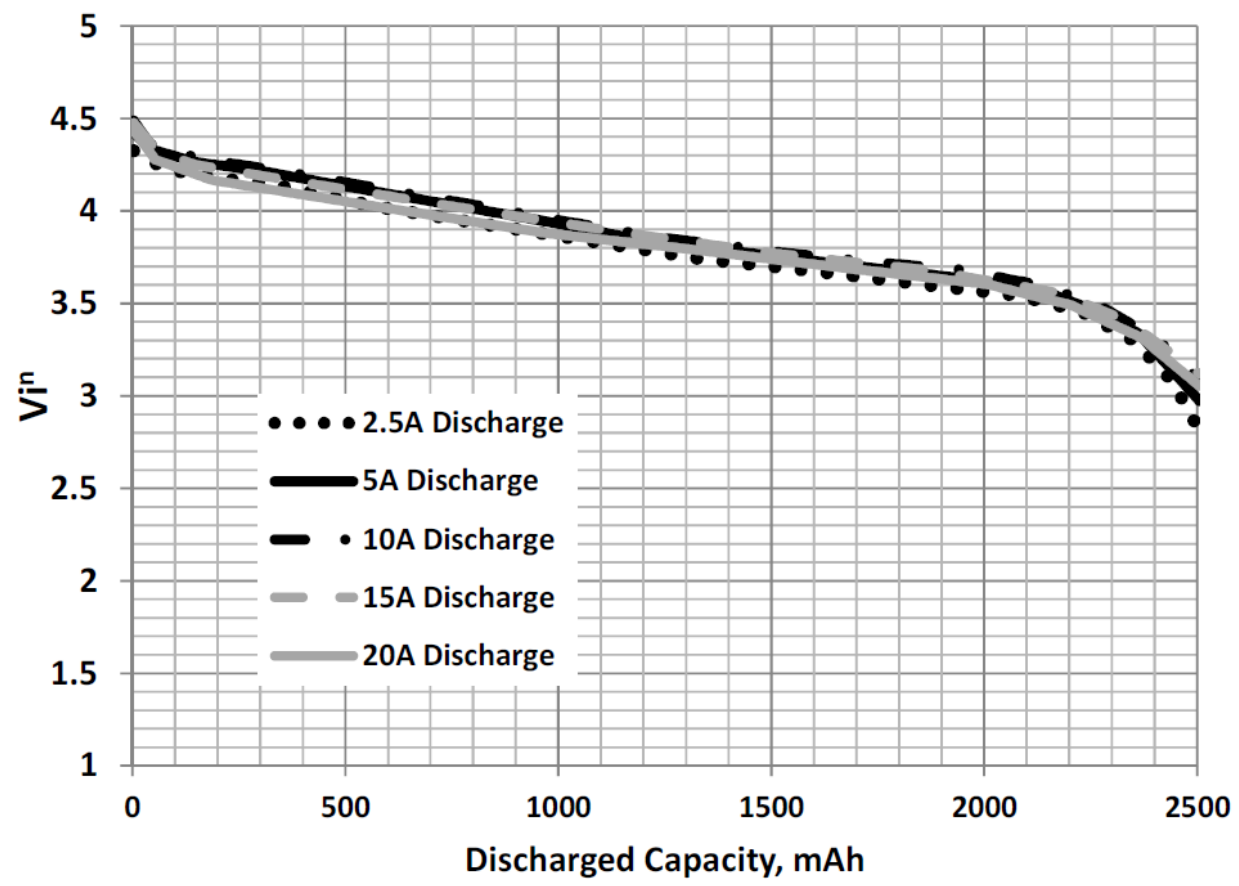

Figure 2.10 INR 18650 correlated current discharge curves. $n=0.05$ and $C=2500 \mathrm{mAh}$ [7] 


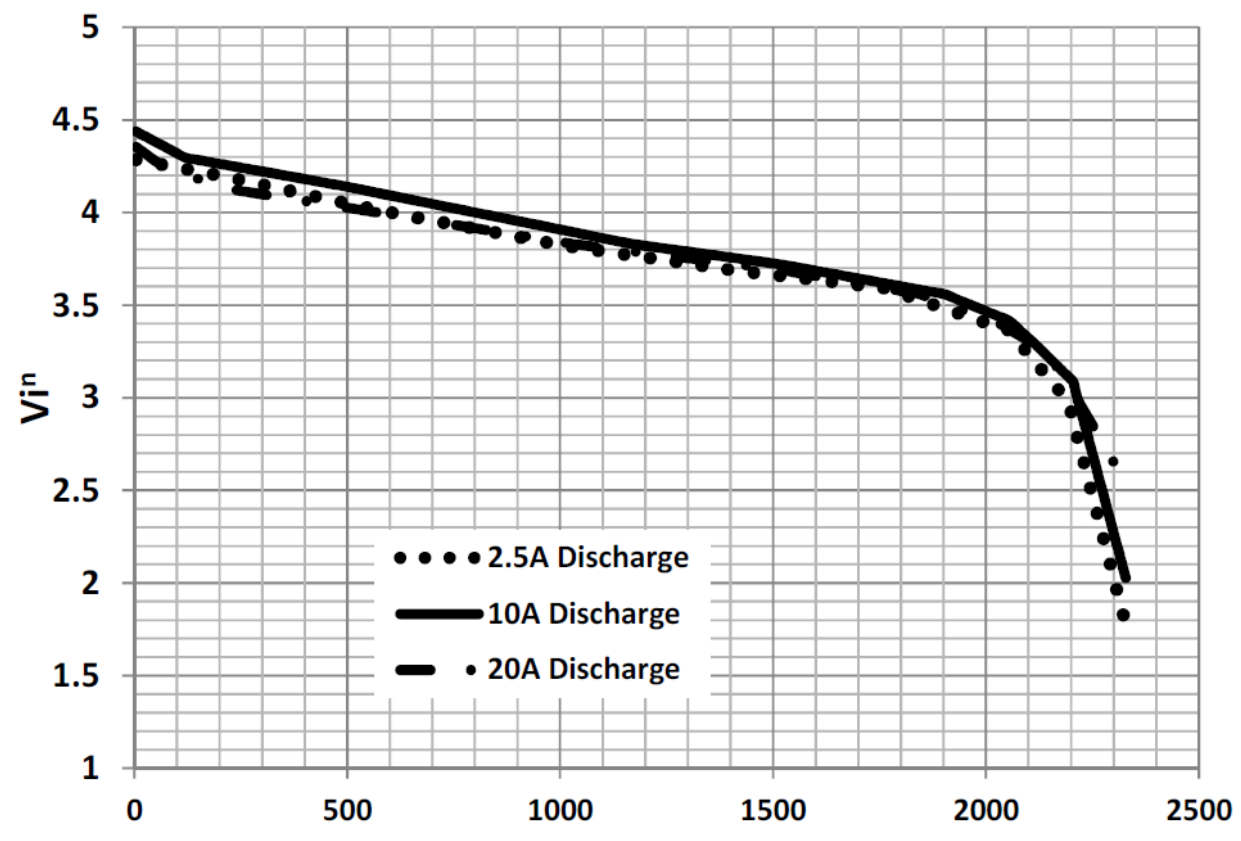

Figure 2.11 LG18B50HE2 correlated current discharge curves $n=0.05$ and $C=2500 \mathrm{mAh}$ [7] 


\section{CHAPTER 3}

\section{UAV TRAJECTORY SIMULATION}

\subsection{Aircraft Equations of Motion}

Mathematical 5-DOF motion equations govern two main characteristics of motion: translational and rotational motion. A total of six equations of motion will be extracted from the investigation of the motion derivatives. In the following section translational motion is analyzed yielding subsequent equations of motion.

\subsubsection{Translational Motion}

Translational motion refers to a motion of aircraft where every line in the body remains parallel to its original position. This is the motion of a rigid body whose mass is concentrated at the center of gravity where other external forces and moments act.

Newton's second law governs the translational equations. Those are uncoupled from the rotational equations for trajectory analyses using the assumptions that rotational rates are small, and the control surface deflections have a negligible effect on forces and non-negligible effect on moments [11]. These types of assumptions are made when fewer than all three Euler angles are used in the simulations (3-DOF,5-DOF) [12].

$$
\text { Translation: } \sum F=m a
$$

Rigid body transformations are calculated from kinematics equations. Those are used to derive the differential equations for motion along the $x, y, z$ axes which locates the aircraft center of gravity relative to the origin of the inertial position (i.e. ground) with the following relation for velocity: 


$$
V=\frac{d \vec{X}}{d t}
$$

The velocity vector $\vec{V}$ and the position vector $\vec{X}$ are later expressed in the same coordinate system to obtain the corresponding scalar equations.

Derivation assumptions for the equations of motion for non-steady flight must be defined before proceeding with the force laws. The following statements must be made for the physical model established for the simulation $[12,11]$ :

a. The earth is flat and non-rotating with an approximate inertial reference frame called the flat earth model.

b. The atmosphere is at rest; atmospheric properties are fixed.

c. Aircraft has fixed engines and a conventional aft tail with right-left plane symmetry.

d. No sideslip forces are acting on the airplane- this implies the aircraft is always flown in coordinated flight. The aircraft is in symmetric flight, and all the aerodynamic forces are acting on the center of gravity of the aircraft.

The following derivation of the equations of motion is defined by the number of coordinates in the system where the aircraft operates. With each coordinate system that moves with the aircraft, the $\mathrm{x}$ and $\mathrm{z}$ axes are in the plane of symmetry of the aircraft, and the $\mathrm{y}$ axis is in the parallel plane to the earth. Lastly, the $\mathrm{z}$ axis points towards the earth given the condition that the aircraft is in an upright orientation. The three Cartesian coordinates form a right-handed coordinate system. The four coordinate systems that are used to derive the equations are defined below: 
a. Ground axes system $x y z$ is fixed to the surface of the earth, and the $x z$ plane is the vertical plane. $\mathrm{z}$ is positive downward; $\mathrm{x}$ and $\mathrm{y}$ follow the right-hand rule. This is also referred to as the inertial reference frame.

b. Local horizon axes system $\boldsymbol{x}_{\boldsymbol{h}} \boldsymbol{y}_{\boldsymbol{h}} \boldsymbol{z}_{\boldsymbol{h}}$ moves with the aircraft and axes are parallel to the ground. Local horizon axes are a translational counterpart of the inertial frame.

c. Wind axes system $\boldsymbol{x}_{\boldsymbol{w}} \boldsymbol{y}_{\boldsymbol{w}} \boldsymbol{z}_{\boldsymbol{w}}$ moves with the aircraft and the $x_{w}$ axis is coincident with the velocity vector. and is tangent to the flight path and positive in forward direction while $y_{w}$ is orthogonal with $x_{w}$ from the clockwise direction in the lateral plane and $z_{w}$ is pointed towards the ground in the vertical axis of the aircraft.

d. Body axes system $\boldsymbol{x}_{\boldsymbol{b}} \boldsymbol{y}_{\boldsymbol{b}} \boldsymbol{z}_{\boldsymbol{b}}$ is a fixed coordinate system to the aircraft. $x_{b}$ is the axis where the aircraft's nose points, $y_{b}$ axis is orthogonal to the $x_{b}$ parallel with the lateral axis, $z_{b}$ is orthogonal to the $y_{b}$ and in the intersecting plane between the body and wind axis similar to the $y_{b}$ axis. 


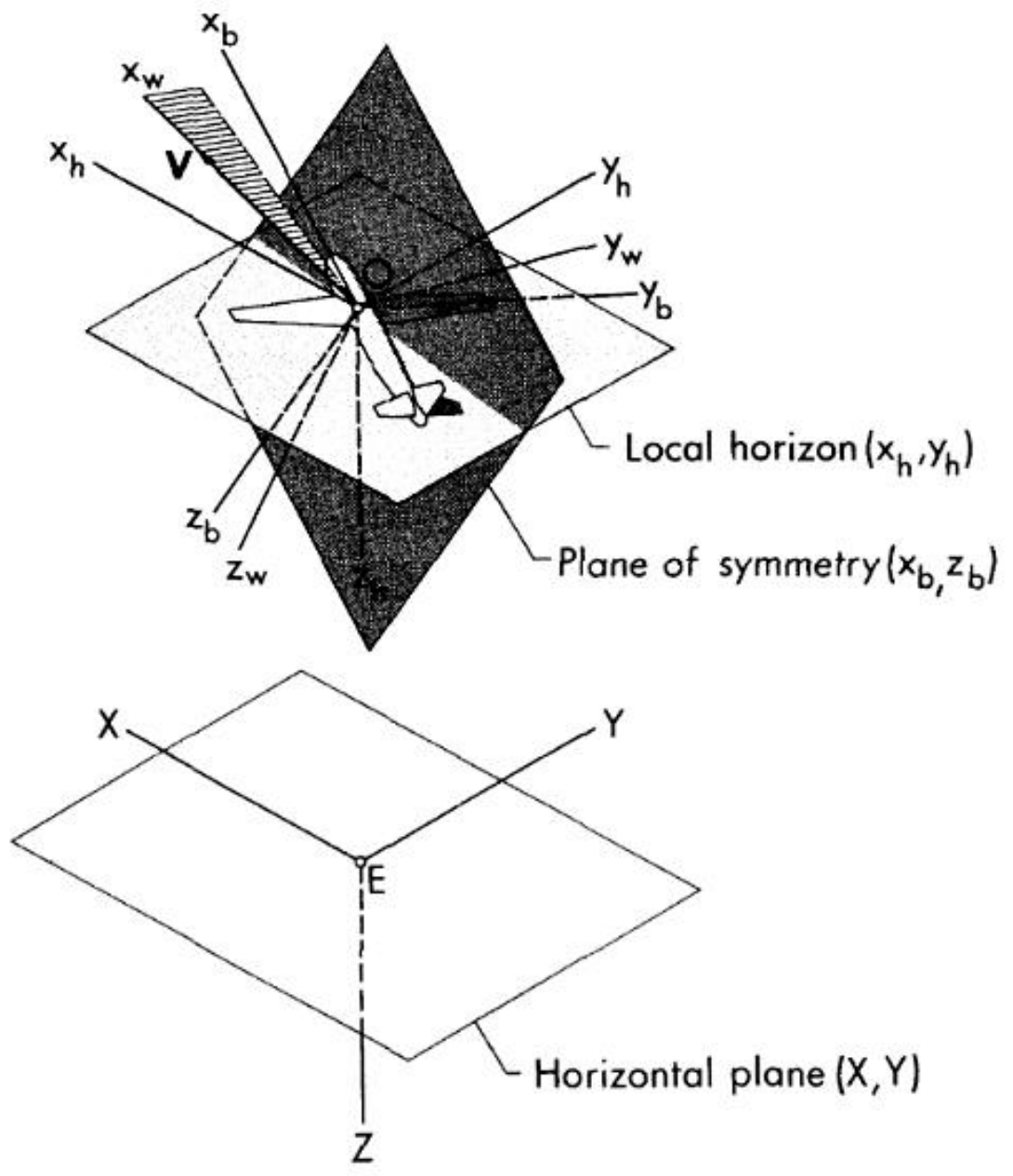

Figure 3.1 Coordinated systems over Flat Earth model

\subsubsection{Coordinate Transformations}

Transformations can be obtained with orthogonality using rotations of velocity yaw $(\beta)$, velocity $\operatorname{pitch}(\theta)$, velocity $\operatorname{roll}(\mu)$ [12]. In order to define these rotations, it is necessary to introduce at least two coordinate systems. In the case that is defined for this simulation, wind axis 
transformation is appropriate. The procedure to obtain those coordinate transformations is to first obtain the partial transformations in matrix form for each dimension of the space [11].

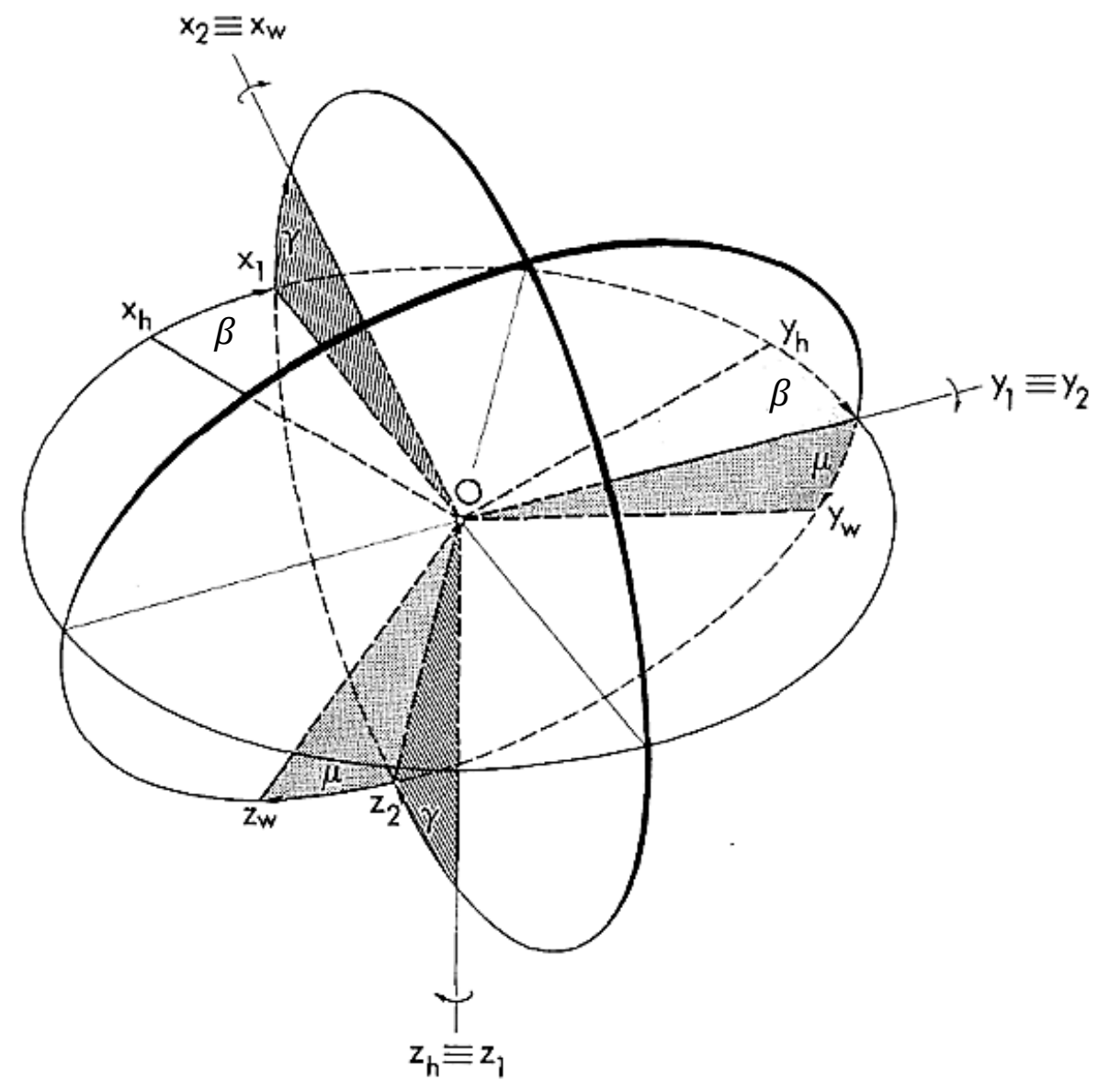

Figure 3.2 Rotations from horizon to the wind axes

From Figure 3.2 the transformation can be exploited with two steps and final transformation matrix from the local horizon axes system to the wind axes system has been obtained as shown: 


$$
\left[\begin{array}{l}
\boldsymbol{i}_{w} \\
\boldsymbol{j}_{w} \\
\boldsymbol{k}_{w}
\end{array}\right]=\left[\begin{array}{ccc}
\cos \gamma \cos \beta & \cos \gamma \sin \beta & -\sin \gamma \\
\sin \mu \sin \gamma \cos \beta-\cos \mu \sin \beta & \sin \mu \sin \gamma \sin \beta+\cos \mu \cos \beta & \sin \mu \cos \gamma \\
\cos \mu \sin \gamma \cos \beta+\sin \mu \sin \beta & \cos \mu \sin \gamma \sin \beta-\sin \mu \cos \beta & \cos \mu \cos \gamma
\end{array}\right]\left[\begin{array}{l}
\boldsymbol{i}_{h} \\
\boldsymbol{j}_{h} \\
\boldsymbol{k}_{h}
\end{array}\right]
$$

There is one more straight transformation necessary to complete angular relationships between the coordinate system used in this simulation. It is simply an equality between local horizon axes and the ground axes which are always parallel; the following relationship can be established:

$$
\left[\begin{array}{l}
\boldsymbol{i}_{h} \\
\boldsymbol{j}_{h} \\
\boldsymbol{k}_{h}
\end{array}\right]=\left[\begin{array}{l}
\boldsymbol{i}_{e} \\
\boldsymbol{j}_{e} \\
\boldsymbol{k}_{e}
\end{array}\right]
$$

where the subscript $e$ refers to the ground system.

The following representation follows the same transformation of coordinate systems with unit vectors indicating the transformation between local and ground coordinate systems defined above:

$$
\begin{gathered}
V=V \cos \gamma \cos \beta \boldsymbol{i}_{e}+V \cos \gamma \sin \beta \boldsymbol{j}_{e}-V \sin \gamma \boldsymbol{k}_{e} \\
\vec{X}=x \boldsymbol{i}_{e}+y \boldsymbol{j}_{e}-z \boldsymbol{k}_{e}
\end{gathered}
$$

It is obtained for the global (inertial) coordinate system and can be converted to the same coordinates,

$$
\begin{gathered}
i_{h}=\boldsymbol{i}_{e} \\
j_{h}=\boldsymbol{j}_{e} \\
k_{h}=\boldsymbol{k}_{e}
\end{gathered}
$$

This way unit vectors $i_{h}$ and $k_{h}$ indicate the local horizontal system and $j_{h}$ is the local vertical system substituted into Eq. (3.5):

$$
V=V \cos \gamma \cos \beta \boldsymbol{i}_{\boldsymbol{h}}+V \cos \gamma \sin \beta \boldsymbol{j}_{\boldsymbol{h}}-V \sin \gamma \boldsymbol{k}_{\boldsymbol{h}}
$$




$$
\vec{X}=x \boldsymbol{i}_{\boldsymbol{h}}+y \boldsymbol{j}_{v}-z \boldsymbol{k}_{\boldsymbol{h}}
$$

Eq. (3.5) becomes after substitution to the differential equation:

$$
V \cos \gamma \cos \beta \boldsymbol{i}_{\boldsymbol{h}}-V \sin \gamma \boldsymbol{k}_{\boldsymbol{h}}+V \cos \gamma \sin \beta \boldsymbol{j}_{\boldsymbol{v}}=\dot{x} \boldsymbol{i}_{\boldsymbol{h}}+\dot{y} \boldsymbol{j}_{v}-\dot{z} \boldsymbol{k}_{\boldsymbol{h}}
$$

This relation leads to the following scalar equations [23]

$$
\begin{gathered}
\dot{x}=V \cos \gamma \cos \beta \\
\dot{y}=V \cos \gamma \sin \beta \\
\dot{z}=V \sin \gamma
\end{gathered}
$$

Translational kinematic equations of motion for the horizontal and vertical plane are obtained with these calculations.

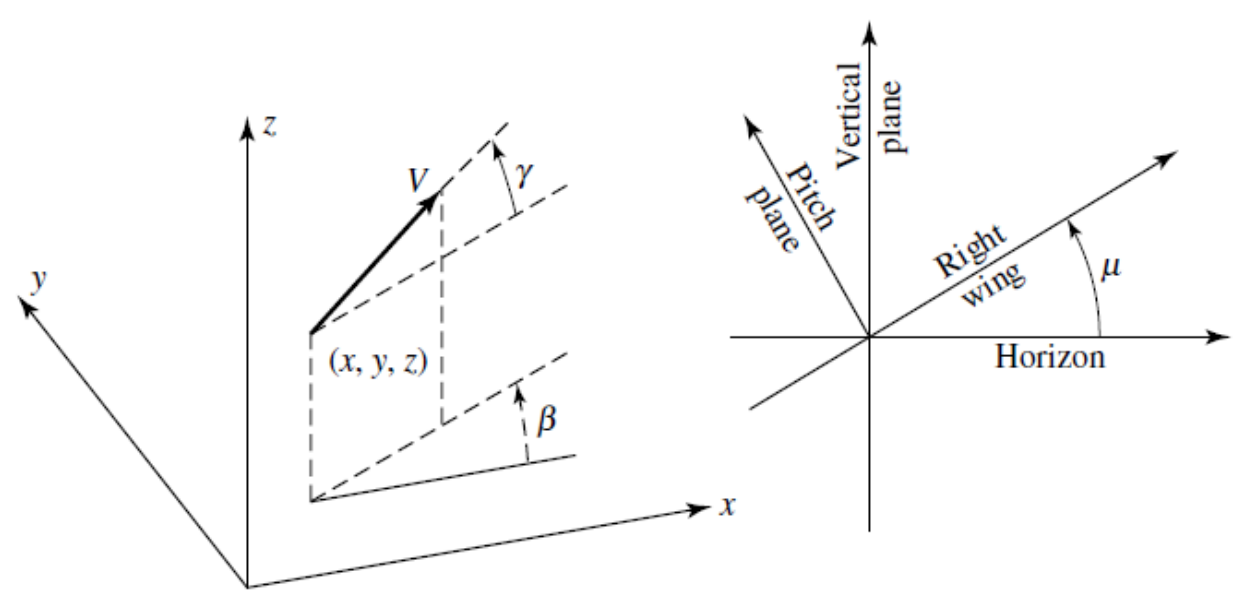

Figure 3.3 Local Coordinate system with Euler angle definitions [23] 


\subsubsection{Rotational Motion}

Establishing rotational equations is a little more involved than scalar translational motions. Equations for $V, \gamma, \beta$ define the velocity vector of the aircraft at the center of gravity relative to the ground. From Newton's second law described in Eq. (3.1), resultant force $F$ acting on the aircraft can be written for the flight condition and expressed with several component forces acting on the aircraft at any point in time. Taking the reference frame fixed to the earth, acceleration $(\vec{a})$ is approximated by the aircraft's acceleration relative to the ground.

The total force acting is,

$$
\overrightarrow{\boldsymbol{F}}=\overrightarrow{\boldsymbol{T}}+\overrightarrow{\boldsymbol{A}}+\overrightarrow{\boldsymbol{W}}
$$

where $\mathrm{T}$ is the thrust, $\mathrm{A}$ is the aerodynamic force. And $\mathrm{W}$ is the weight of the aircraft. These forces integrated at the center of gravity of the aircraft which creates the appropriate moments. Note that for 5-DOF motion analysis it is not necessary to calculate moments additionally to the motion derivatives since force and moment equations are uncoupled.

The aerodynamic force is divided into two main components $\operatorname{Lift}(L)$ and $\operatorname{Drag}(D)$ as follows,

$$
\vec{A}=\vec{D}+\overrightarrow{\boldsymbol{L}}
$$

Similar to the translational equation calculation, it is necessary to identify the coordinate system. Instead of using the local coordinates a more direct derivation can be performed with dynamic equations using the wind axes system. Note that there is no side slip force acting on the aircraft (y direction). The wind axes system notation of the thrust, lift, drag, and weight formulation can be found as follows: 


$$
\begin{gathered}
\overrightarrow{\boldsymbol{T}}=T \cos \alpha \boldsymbol{i}_{w}-T \sin \alpha \boldsymbol{k}_{w} \\
\overrightarrow{\boldsymbol{D}}=-D \boldsymbol{i}_{w} \\
\overrightarrow{\boldsymbol{L}}=-L \cos \mu \boldsymbol{k}_{w}+L \sin \mu \boldsymbol{j}_{w} \\
\overrightarrow{\boldsymbol{W}}=-W \sin \gamma \boldsymbol{i}_{w}+W \cos \gamma \boldsymbol{k}_{w}
\end{gathered}
$$

Combining these force formulations into resultant external forces,

$$
\overrightarrow{\boldsymbol{F}}=(T \cos \alpha-D-W \sin \gamma) \boldsymbol{i}_{w}+(L \sin \mu) \boldsymbol{j}_{w}-(T \sin \alpha+L \cos \mu-W \cos \gamma) \boldsymbol{k}_{w}
$$

The acceleration definition relative to the ground can be written

$$
a=\frac{d \overrightarrow{\boldsymbol{V}}}{d t}
$$

Integrating velocity along the $x_{w}$ axis yields the following relation [24]

$$
\begin{gathered}
\overrightarrow{\boldsymbol{V}}=V \boldsymbol{i}_{w} \\
a=\frac{d \boldsymbol{V}}{d t}=\dot{V} i_{w}+V \frac{d i_{w}}{d t}
\end{gathered}
$$

Relation obtained for $i_{w}$ from the Eq. (3.3):

$$
\boldsymbol{i}_{w}=\cos \gamma \cos \beta \boldsymbol{i}_{h}+\cos \gamma \sin \beta \boldsymbol{j}_{h}-\sin \gamma \boldsymbol{k}_{h}
$$

Derived respect to time,

$$
\begin{array}{r}
\frac{d i_{w}}{d t}=-\boldsymbol{i}_{h} \dot{\beta} \sin \beta \cos \gamma+\boldsymbol{j}_{h} \dot{\beta} \cos \beta \cos \gamma-\boldsymbol{i}_{h} \cos \beta \dot{\gamma} \sin \gamma \\
-\boldsymbol{j}_{h} \sin \beta \dot{\gamma} \sin \gamma-\boldsymbol{k}_{h} \dot{\gamma} \cos \gamma
\end{array}
$$

and rearranged as

$$
\begin{gathered}
\frac{d i_{w}}{d t}=-\boldsymbol{i}_{h}(\dot{\beta} \dot{\gamma} \sin \beta \cos \gamma \cos \beta \sin \gamma)+\boldsymbol{j}_{h}(\dot{\beta} \cos \beta \cos \gamma-\dot{\gamma} \sin \beta \sin \gamma) \\
-\boldsymbol{k}_{h}(\dot{\gamma} \cos \gamma)
\end{gathered}
$$


We solve the Matrix inverse of the Eq. (3.3)

$$
\left[\begin{array}{l}
\boldsymbol{i}_{h} \\
\boldsymbol{j}_{h} \\
\boldsymbol{k}_{h}
\end{array}\right]=\left[\begin{array}{ccc}
\cos \gamma \cos \beta & \sin \mu \sin \gamma \cos \beta-\cos \mu \sin \beta & \cos \mu \sin \gamma \cos \beta+\sin \mu \sin \beta \\
\sin \beta \cos \gamma & \sin \mu \sin \gamma \sin \beta+\cos \mu \cos \beta & \cos \mu \sin \gamma \sin \beta-\sin \mu \cos \beta \\
-\sin \gamma & \cos \gamma \sin \mu & \cos \mu \cos \gamma
\end{array}\right]\left[\begin{array}{l}
\boldsymbol{i}_{w} \\
\boldsymbol{j}_{w} \\
\boldsymbol{k}_{w}
\end{array}\right]
$$

From the combination of Eq. (3.21), Eq. (3.24) and Eq. (3.25) the following dynamics relations for Mathematical 5-DOF [23] dynamic systems are obtained:

$$
\begin{gathered}
\dot{V}=\frac{1}{m}[T \cos \alpha-D]-g \sin \gamma \\
\dot{\beta}=\frac{1}{m V}[T \sin \alpha+L](\sin \mu / \cos \gamma) \\
\dot{\gamma}=\frac{1}{m V}[T \sin \alpha+L] \cos \mu-\left(\frac{g}{V}\right) \cos \gamma
\end{gathered}
$$

The control parameters are:

$$
C L(\alpha), \mu, T
$$

The take-off stage calculation requires a similar but slightly modified version of the velocity derivative in order to account for runway friction effects, and its derivation is given in reference [25].

$$
\left.\dot{V}=\frac{g}{W}[T-D-\xi(W \cos \gamma-L)-W \sin \gamma)\right]
$$

where $\xi$ is the runway friction coefficient. Its value for the non-braking situation ranges from 0.020.08. In the case of our investigation it takes " 0.04 " which is the empirical value given for the dry asphalt [25]. In Figure 3.4 take-off schematic is demonstrated with the acting forces on the aircraft at the ground. 


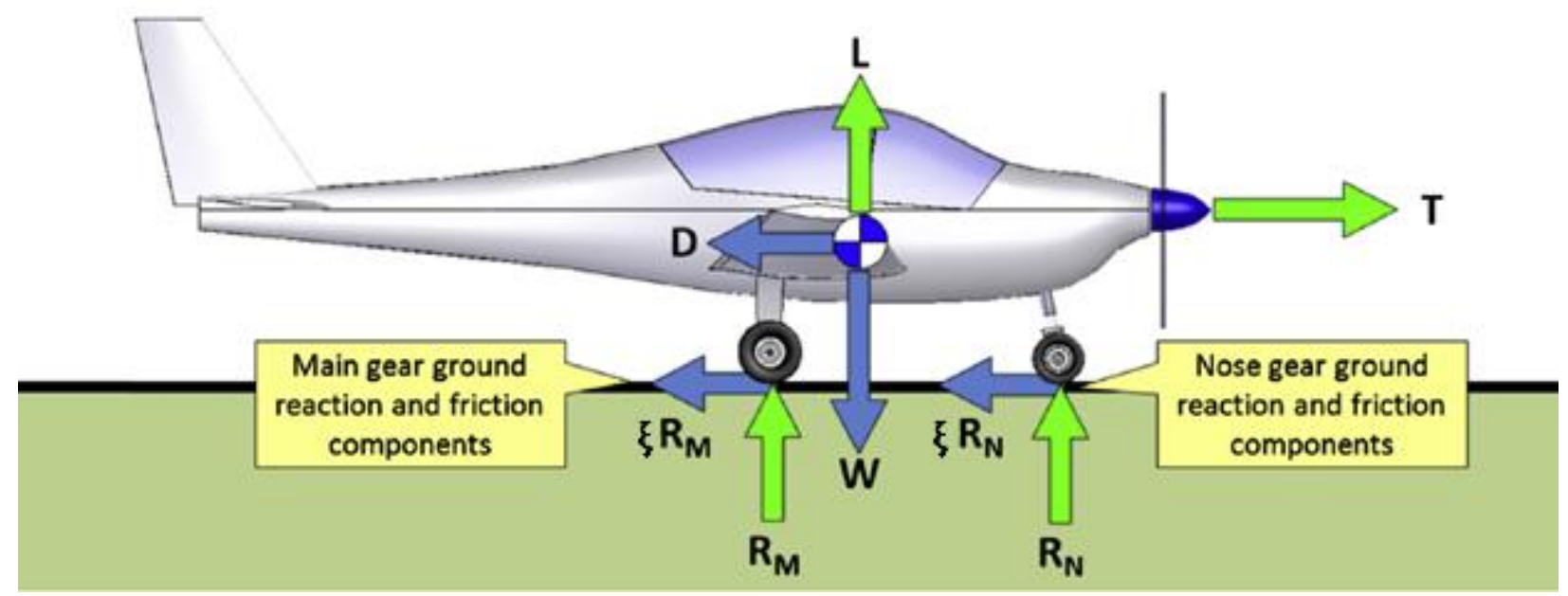

Figure 3.4 Balanced take-off free-body diagram for tricycle landing gear configuration [26]

\subsection{Solver Method}

\subsubsection{Runge-Kutta-Fehlberg Method with Adaptive Step-Size}

One of the biggest considerations while deciding on an ODE solver for the simulation algorithm is to opt for a solver that is capable of solving the three ODEs provided in a relatively efficient way, while still within the reliable limits of the differential equation solution. This is largely a function of the step size selected for the simulation depending on the accuracy of the solver.

From the previous section, degrees of freedom analysis systems consist of non-stiff mathematical initial value problems, so solving them would require correct numerical method in order to get the best efficiency and accuracy from the numerical investigation. Hence, the RungeKutta family method has been adopted to solve the system generated in the previous sub-section. 
Adaptive methods for Runge-Kutta are designed to produce an estimate of the Runge-Kutta step and the local truncation error. This is done by implementing two methods, one for order $\boldsymbol{p}$ and another with $\boldsymbol{p}$-1. These methods have an advantage over the other explicit methods since calculating error does not incur significant computational cost compared to a step with the higherorder methods.

A general formulation for Runge-Kutta-Fehlberg next step solution can be calculated from the following relation [27].

$$
y_{n+1}=y_{n}+h \sum_{i, j=1}^{s, s-1} b_{i j}^{\star} k_{i}
$$

Similarly, truncation error gives

$$
e_{n+1}=y_{n+1}-y_{n+1}^{\star}=h \sum_{i, j=1}^{s, s-1}\left(b_{i j}-b_{i j}^{\star}\right) k_{i}
$$

where $\mathrm{h}$ is the adaptive step size. The butcher tableau is used to find the values for $b_{i j}^{\star}$ [27]. 


\begin{tabular}{|c|c|c|c|c|c|c|c|c|c|c|c|c|c|}
\hline \multirow[b]{2}{*}{$i \bigvee j$} & \multirow[t]{2}{*}{$\alpha_{i}$} & \multicolumn{12}{|c|}{$b_{i j}$} \\
\hline & & 0 & 1 & 2 & 3 & 4 & 5 & 6 & 7 & 8 & 9 & 10 & 11 \\
\hline 0 & 0 & 0 & & & & & & & & & & & \\
\hline 1 & 2 & 2 & & & & & & & & & & & \\
\hline 1 & $\overline{27}$ & $\overline{27}$ & & & & & & & & & & & \\
\hline 2 & 1 & 1 & 1 & & & & & & & & & & \\
\hline 2 & $\overline{9}$ & $\overline{36}$ & $\overline{12}$ & & & & & & & & & & \\
\hline 3 & 1 & 1 & 25 & 1 & & & & & & & & & \\
\hline & $\overline{6}$ & $\overline{24}$ & $\overline{16}$ & $\overline{8}$ & & & & & & & & & \\
\hline 4 & 5 & 5 & 0 & 25 & 25 & & & & & & & & \\
\hline & $\overline{12}$ & $\overline{12}$ & & $\overline{16}$ & $\overline{16}$ & & & & & & & & \\
\hline 5 & 1 & 1 & 0 & 0 & 1 & 1 & & & & & & & \\
\hline & $\overline{2}$ & 20 & & & $\overline{4}$ & $\overline{5}$ & & & & & & & \\
\hline 6 & 5 & 25 & 0 & 0 & 125 & 65 & 125 & & & & & & \\
\hline & $\overline{6}$ & $\overline{108}$ & & & $\overline{108}$ & $\overline{27}$ & $\overline{54}$ & & & & & & \\
\hline 7 & $\frac{1}{-}$ & 31 & 0 & 0 & 0 & 61 & -2 & 13 & & & & & \\
\hline & 6 & 100 & & & & 225 & 9 & $\overline{900}$ & & & & & \\
\hline 8 & $\frac{2}{3}$ & 2 & 0 & 0 & $-\frac{53}{6}$ & 704 & $-\frac{107}{0}$ & $\frac{67}{00}$ & 3 & & & & \\
\hline 9 & 1 & 91 & & & 23 & 976 & 311 & 19 & 17 & 1 & & & \\
\hline y & $\overline{3}$ & $\overline{100}$ & 0 & 0 & $\overline{108}$ & $\overline{135}$ & $\overline{54}$ & $\overline{60}$ & $\overline{6}$ & $\overline{12}$ & & & \\
\hline 10 & 1 & 2383 & 0 & 0 & 341 & 4496 & 301 & 2133 & 45 & 45 & 18 & & \\
\hline 10 & & $\overline{4100}$ & & & $\overline{164}$ & $\overline{1025}$ & $\overline{82}$ & $\overline{4100}$ & $\overline{82}$ & $\overline{164}$ & $\overline{41}$ & & \\
\hline 11 & 0 & 3 & 0 & 0 & 0 & 0 & 6 & 3 & 3 & 3 & 6 & 0 & \\
\hline & & 205 & & & & & 41 & $\overline{205}$ & $\overline{41}$ & $\overline{41}$ & $\overline{41}$ & & \\
\hline 12 & 1 & 1777 & 0 & 0 & 341 & 3396 & 289 & 2193 & 51 & 33 & 12 & 0 & 1 \\
\hline & & 4100 & 0 & 0 & 164 & $\overline{1025}$ & 82 & $\overline{4100}$ & $\overline{82}$ & $\overline{164}$ & 41 & & \\
\hline
\end{tabular}

Table 3.1 Runge-Kutta-Fehlberg 7(8) [8]

Truncation error is estimated to be:

$$
\epsilon_{t}=-\frac{41}{840} \times h \times\left(k_{o}+k_{10}-k_{11}-k_{12}\right)
$$


Adaptive step size for the method is calculated by Dormand and Prince [28] up to the $6^{\text {th }}$ order:

$$
\text { Scale }=0.9 \times\left[\frac{\text { tol } \times y_{i+1}}{\epsilon_{t}}\right]^{\frac{1}{p+1}}
$$

And the next step size is calculated as

$$
h_{n+1}=h_{n} \times \text { Scale }
$$

where $h_{n+1}$ is the next predicted step size that satisfies the selected tolerance "tol" value and $p$ is the order of step size calculation. For this simulation, the step calculation order was chosen as " 6 " which yields the best order possible with the initial value problem solved with the RKF7(8) method.

Pseudo Code for the adaptive step size calculation is shown below: 


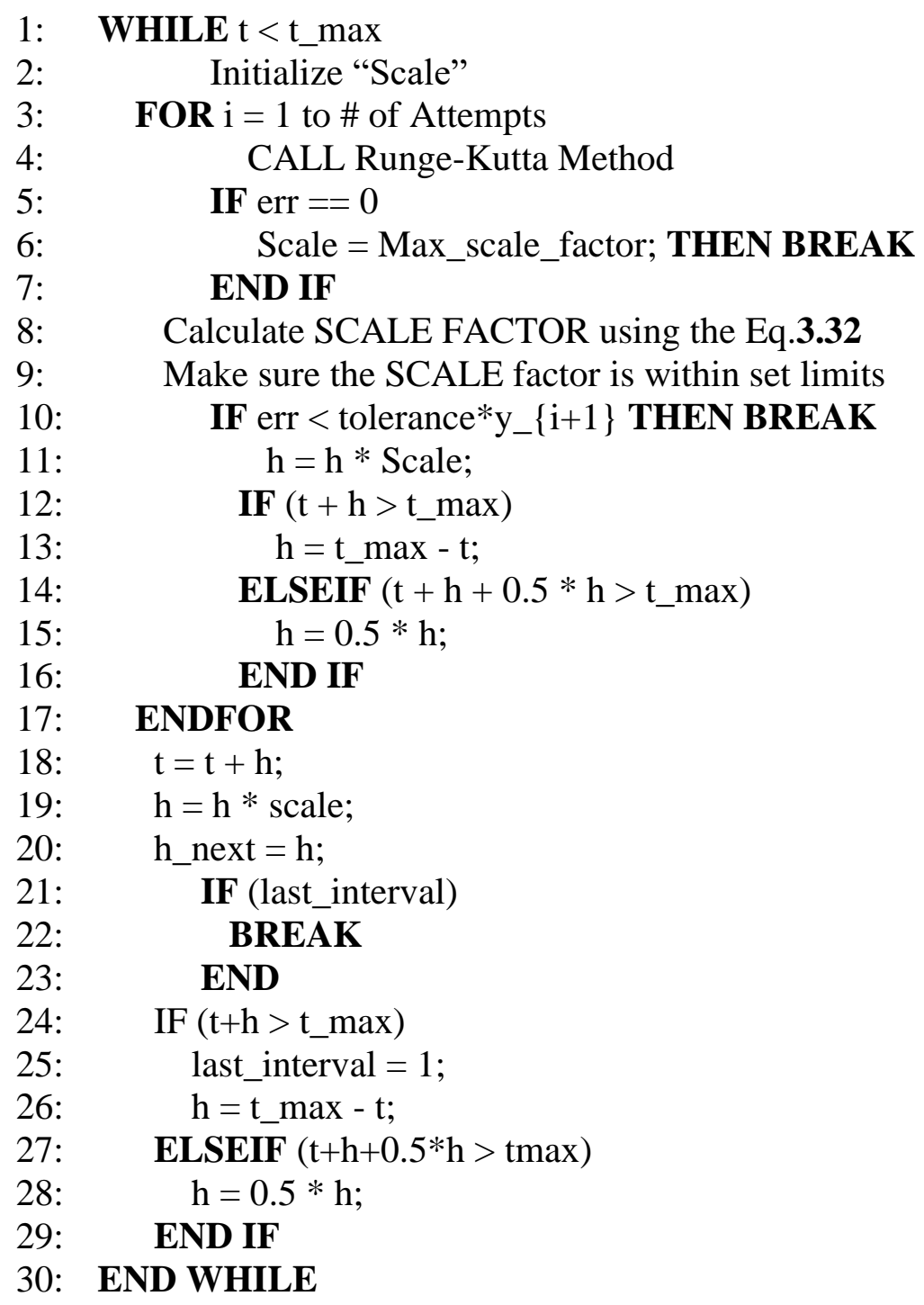

Algorithm 3.1 Pseudo code algorithm for Adaptive-step size [28]

\subsection{BASIC FLIGHT GUIDANCE AND CONTROL}

Flight control systems for trajectory analysis differ from stability and control analysis. Use of the equation of motion defined by the 5-DOF system also assumes coordinated flight 


\subsubsection{Pitch Control}

Alternating the pitch angle will control aircraft longitudinal attitude from the horizon and it is a necessary control parameter for the Mathematical 5-DOF simulation. Aircraft angles representing the longitudinal movement are related as

$$
\theta=\gamma+\alpha
$$

where $\gamma$ is flight path angle, $\alpha$ is the angle of attack and the sum yields $\theta$ which is pitch angle. Knowing from Eq. (3.25), angle of attack is one of the inherent control parameters of the Mathematical 5-DOF system. In the case of simplified control where there are no control surfaces accounting for changes to the forces and moments, the only control action that can be created is by manipulating the simulation control parameters directly. Since angle of attack could be adjusted in the simulation and initial values of flight path angle calculated, commanded pitch angle dictates the angle attack of the system.

By this means of control, the altitude of the aircraft can be computed through the equation of longitudinal motion since there is no noise in the simulation. First, the required lift coefficient for the desired flight angle necessary is calculated with the following formulation:

$$
C L_{c}=\frac{\left(\left(\left(\gamma_{c}-\gamma\right) h+\left(\frac{g}{V}\right) \cos \gamma\right)\left(\frac{m V}{\cos \mu}\right)-T \sin \alpha\right)}{q_{\infty} V^{2}}
$$

where $\mathrm{h}$ is the step time of the simulation and angle of attack is a function of the lift coefficient and can be solved from the lift lookup table:

$$
\alpha_{c}=f\left(C L_{c}\right)
$$

From Eq. (3.32), the commanded pitch angle can be found: 


$$
\theta_{c}=\gamma+\alpha_{c}
$$

Commanded flight path angle $\gamma_{c}$ is estimated for smooth transition to the cruise phase following the climb phase when $h \geq 0.95 h_{\text {cruise }}$ is satisfied:

$$
\gamma_{c}=\frac{\left(h_{\text {cruise }}-\text { zcoor }\right)}{i}
$$

where $i$ is the index which controls the magnitude of the control factor until $\gamma_{c}<\gamma$ is satisfied.

\subsubsection{Heading Control}

Directional control in the $x_{h}-y_{h}$ plane can be implemented by manipulating the rolling angle as a factor in the heading EOM. The resultant rate of heading change for a coordinated turn is given by the rate of turn equation [29]

$$
\dot{\psi}=\frac{g \tan \phi}{U_{\infty}},
$$

Solving for bank angle yields

$$
\phi \approx \frac{U_{\infty} \dot{\psi}}{g}
$$

This is obtained with the assumption of $\phi \ll 1[29]$. However, in real conditions turn rate is a very noisy signal when based on bank angle; hence, the generation of a smoother signal should be created by filtering the equation. If the desired heading is known and selected as $\psi_{d}$ and the objective is to obtain the $\psi$ to follow the $\psi_{d}$ relatively slowly, the dynamics of the equation are expressed as [29]:

$$
\begin{gathered}
\tau_{1} \dot{\psi}+\psi=\psi_{d} \\
\frac{\psi}{\psi_{d}}=\frac{1}{\tau_{1} s+1}
\end{gathered}
$$


Depending on the objective, $\tau_{1}$ generally gets a value between $15-20 \sec$ [29]. In this thesis it is selected as $20 \mathrm{sec}$ since it yields the smoothest turn rate change. Finally, the desired bank angle is obtained with the filtering [29]:

$$
\begin{gathered}
\dot{\psi}=\frac{1}{\tau_{1}}\left(\psi_{d}-\psi\right), \\
\phi_{d}=\frac{U_{\infty}}{g} \dot{\psi}=\frac{U_{\infty}}{\tau_{1} g}\left(\psi_{d}-\psi\right)
\end{gathered}
$$

This equation is solved through the simulation for each time step. When the turn has commenced according to the flight plan, this would take effect and provide the desired bank angle. The algorithm selects the bank angle for the next time step. If the desired time step requires a higher than feasible banking rate, then the maximum banking rate limit is utilized until it is feasible again.

\subsection{Aircraft Propulsion and Aerodynamics}

Construction of aircraft systems is the crucial part of the performance calculation. In order to get the best accuracy possible sub-systems of the aircrafts must be either modelled accurately, or they can be generated with the help of external software or tools and integrated as lookup tables into the solver's directory. Since the main objective is not to focus on every individual sub-system that conventional electric aircraft can have, instead exploiting the various tools for each important calculation model the source code are able to read the pre-calculated table values from the source directory. The following modules describe the two sub systems and how they are represented in the source code. 


\subsubsection{Propulsion Modeling}

Propulsion systems of Electric powered aircraft mainly influenced battery size and components for power transmission. In the sample aircraft "AVISTAR RC", the propulsion system is comprised of battery, motor, and propeller. There is also an electronic speed controller (ESC) and power cables connecting the electric powertrain together which contribute to the overall efficiency of the system.

The propulsion model explains the power transmission from battery power to thrust power. In a high-level diagram shown in Figure 3.5, batteries provide the propulsion power. Then, the motor converts the power into the rotational power to drive the propeller. As with any powerenergy conversion this will result in some efficiency loss from the overall system. This is called the efficiency loss due to motor. After the rotational energy is converted by the motor, the propeller converts the yielding energy to the thrust forces by simply rotating. This causes an efficiency loss. From the nature of this loss source it's called efficiency due to propeller. In the following diagram [6], the powertrain of the fully electric aircraft has been illustrated with the efficiency losses caused by each component of the powertrain system. 


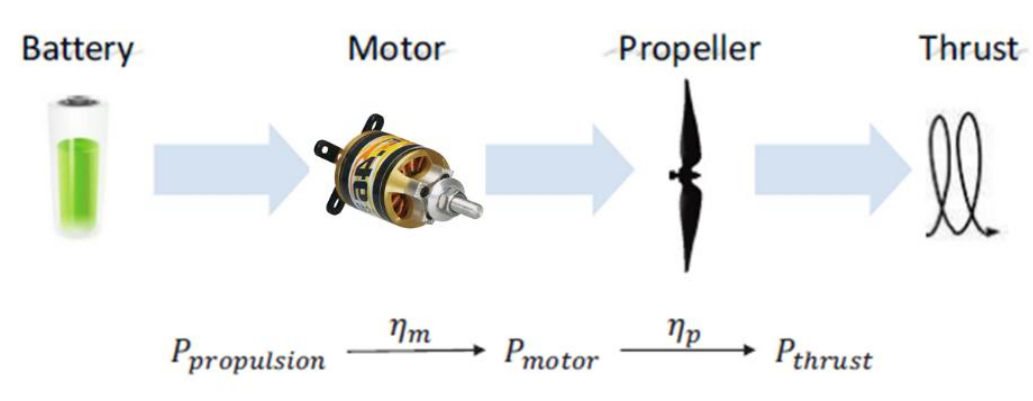

Figure 3.5 High-level diagram of the propulsion model

The next step is to investigate the parts of the propulsion system in detail and demonstrate the methods used in this thesis for each stage. The battery simulation and estimation will be explained in the next chapter in depth. The motor from the powertrain is investigated in this section.

The motor used in the sample plane is a Rimfire .46 Brushless Motor, and specifications [30] are shown in Table 3.2.

\begin{tabular}{|c|c|}
\hline No load current $\left(\boldsymbol{i}_{\mathbf{0}}\right)=$ & $\mathbf{4 . 6 A}$ \\
\hline $\boldsymbol{K}_{\boldsymbol{v}}$ rating $=$ & $800 \mathrm{Kv}$ \\
\hline Motor internal resistance $(\boldsymbol{R})=$ & $0.04 \mathrm{ohms}$ \\
\hline Motor terminal voltage $\left(\boldsymbol{V}_{\boldsymbol{m}}\right)=$ & $V_{t} \times t_{\text {power }}$ \\
\hline Shaft Rotation $(\Omega)=$ & $8000 R P M$ \\
\hline
\end{tabular}




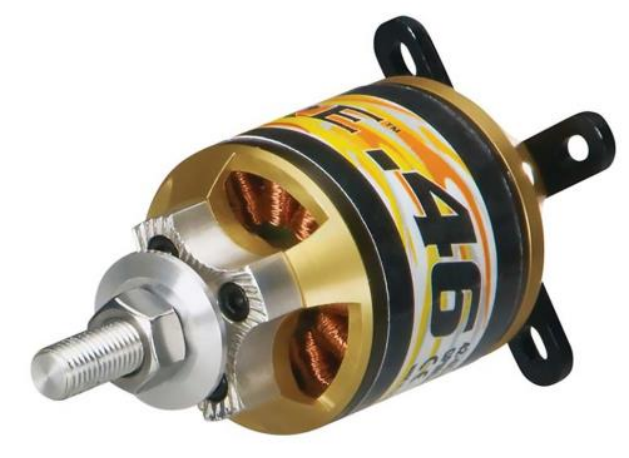

Figure 3.6 Great Planes Rimfire .46-60-800 Outrunner Brushless Motor

The motor efficiency $\eta_{m}$, for any brushless DC-motor can be calculated analytically using the motor terminal voltage $V_{m}$ and the shaft rotation rate $\boldsymbol{\Omega}$ relation. A first order approximation [31] is determined as

$$
\eta_{m}\left(\Omega, V_{m}\right)=\left(1-\frac{i_{0} R}{V_{m}-\frac{\Omega}{K_{v}}}\right) \times \frac{\Omega}{V_{m} K_{v}}
$$

The validation of this function can be performed when the parameters that make the equation are known. From Table 3.2 parameters are obtained. While writing the voltage drawn from the motor, the following expression is needed:

$$
V_{m}=V_{t} \times t_{\text {power }}
$$

where $t_{\text {power }}$ is the throttle percentage command by the ESC controller and can be approximated from the RPM value of the propeller by knowing the capabilities of the battery such as the 
maximum current load of the battery system. Assuming it is linearly changing, throttle level value is estimated for 8000RPM turn rate at the motor with the configuration described as

$$
t_{\text {power }}=0.47
$$

Assuming the base voltage level comes from the 6-cell battery used (PULSE 3300mah 45C 6S LiPo), this yields the following equation for base terminal voltage and the motor terminal voltage. Knowing each individual cell voltage,

$$
V_{t}=4.2 \times 6=25.2 \mathrm{~V}
$$

and

$$
V_{m}=25.2 \times 0.47=11.844 \mathrm{~V}
$$

Now, substituting the values from Table 3.2 and using Eq. (3.49) in Eq. (3.45) yields the following solution for motor efficiency:

$$
\eta_{m}=0.7601
$$

This calculation is carried out throughout the simulation to obtain the dynamic efficiency level changing with the RPM and voltage for the motor.

The next step for propulsion systems modelling is to obtain the propeller parameters and calculate the performance from the propeller performance coefficients. The accepted definition of those parameters is given in reference [32, 33, 34];

$$
\begin{aligned}
& C_{T}=\frac{T}{\rho n^{2} D^{4}} \\
& C_{P}=\frac{P}{\rho n^{3} D^{5}}
\end{aligned}
$$

where $\mathrm{n}$ is the rotational speed in revolutions per second $(R P M / 60 \mathrm{sec})$ and $D$ is the propeller diameter. Other parameters must be in consistent units in order to reach the correct values. 
The advance ratio is defined as

$$
J=\frac{V}{n D}
$$

Knowing the $J$ and having calculated $C_{P}, C_{T}$, one can now calculate the propeller efficiency as

$$
\eta_{P}=\frac{T V}{P}=\frac{C_{T} J}{C_{P}}
$$

The combined relations obtained from blade element theory enables the determination of the required performance graphs of the given propeller for an aircraft.

From the manufacturer sources [33] $A P C 12 \times 6 E$ the propeller is chosen for the sample aircraft, and the following graphs are given for the propeller.

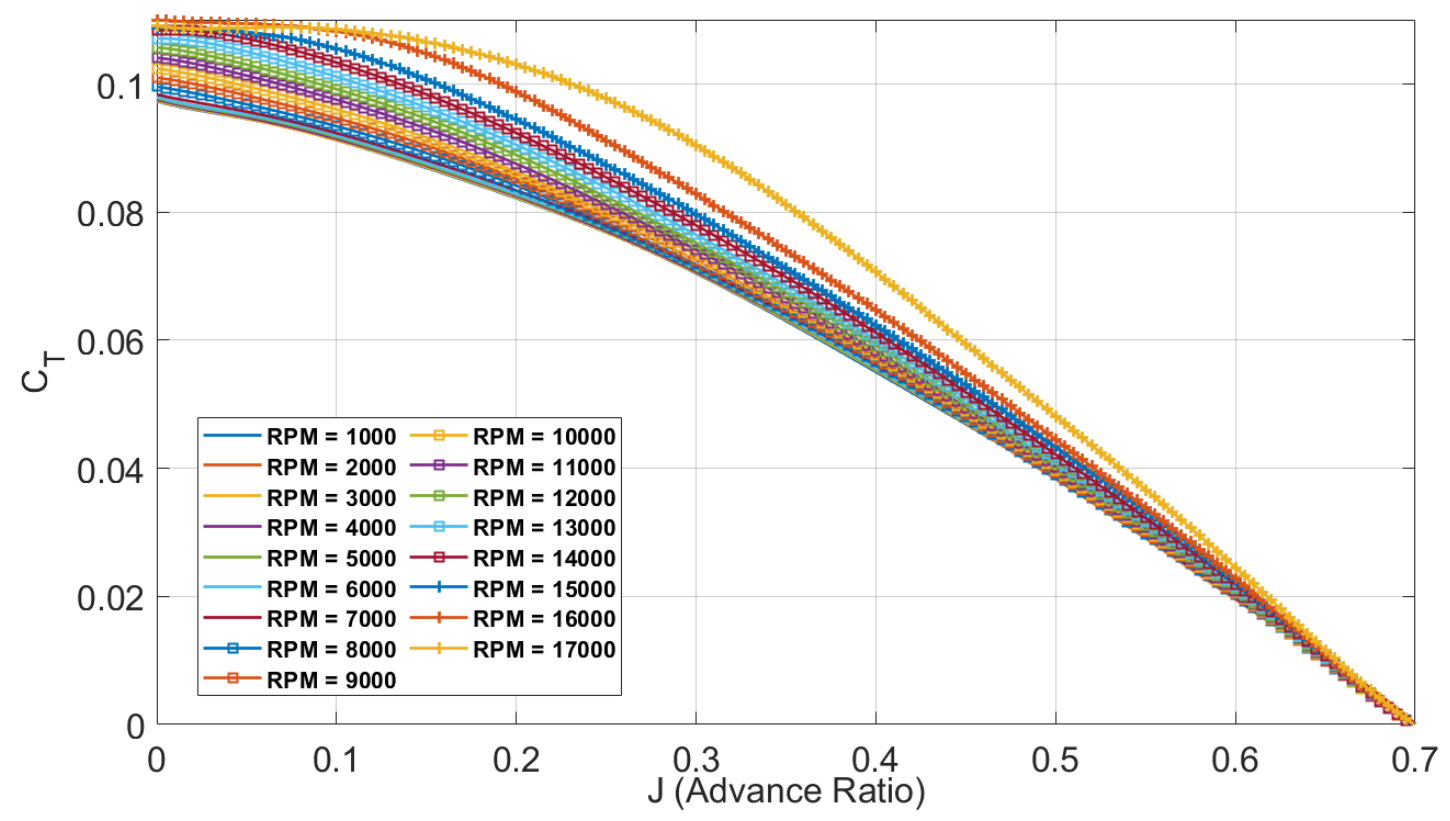

Figure $3.7 C_{T}$ vs J graph for APC $12 x 6$ E propeller RPM ranging 1000 to 17000 


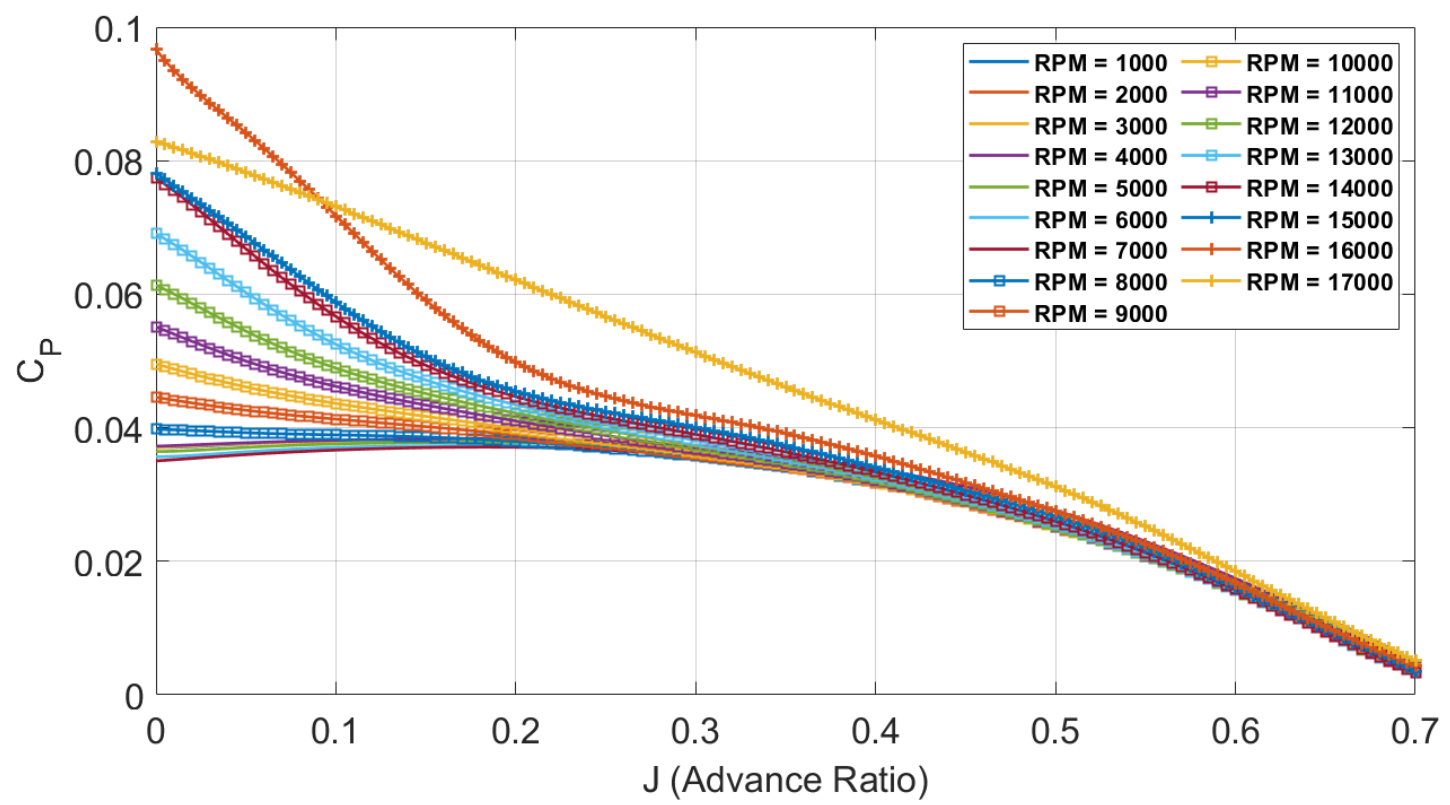

Figure 3.8 $C_{P}$ vs J graph for APC $12 x 6 E$ propeller RPM ranging 1000 to 17000 


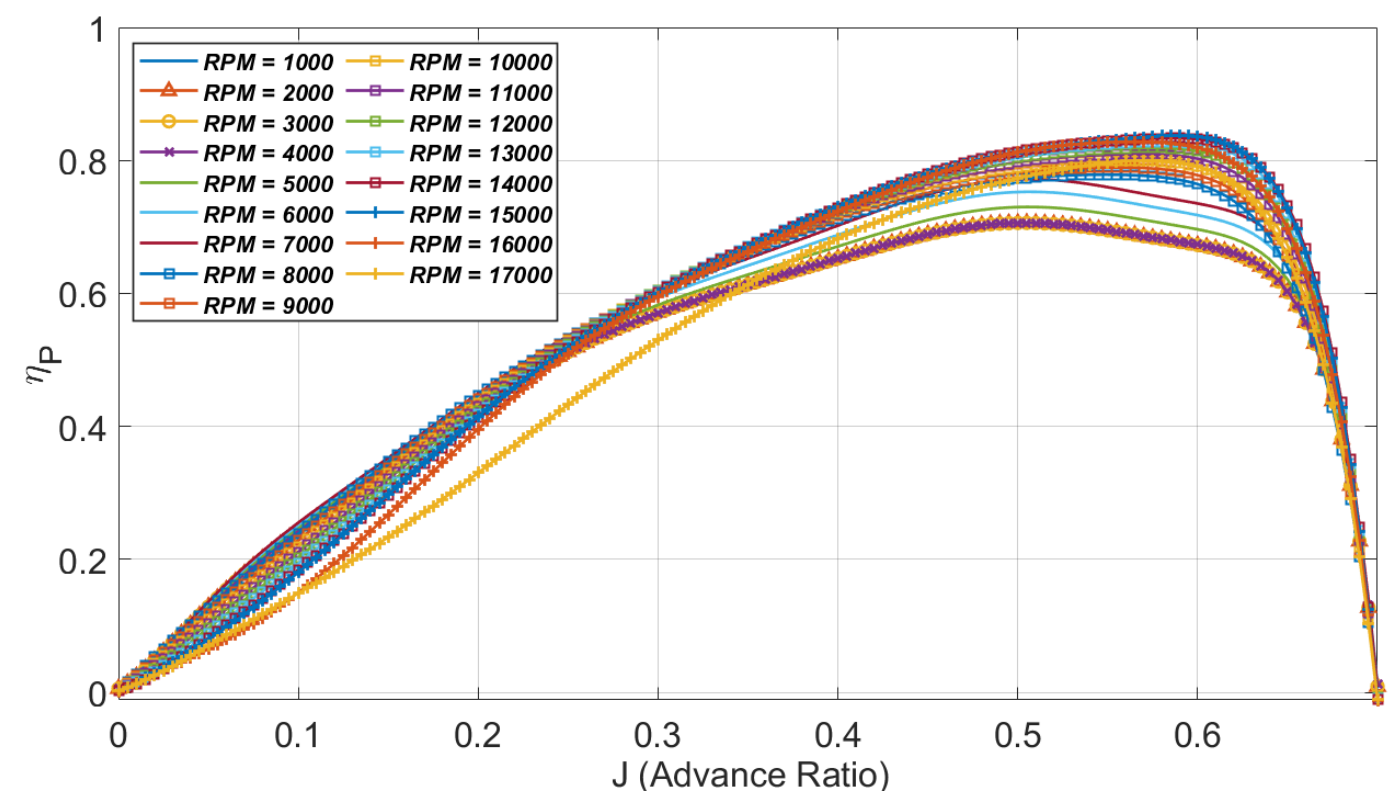

Figure $3.9 \eta_{P}$ vs J graph for APC $12 x 6 E$ propeller RPM ranging 1000 to 17000

\subsubsection{Aerodynamic Investigation}

Aerodynamic coefficients of the aircraft are calculated using several methods and tools. Opting for the right tools is an important factor of the flight simulation. For this simulation the combination of empirical formulas is from aircraft design textbooks and an analysis tool called XFLR5 [35].

XFLR5 is the current iteration of a design and analysis program called XFOIL that was developed by "Mark Drela" at MIT as a design tool for the "MIT Daedalus" project in the 1980s [36]. A user-friendly interface and new 3-D capabilities make it a very useful tool for engineering 
projects. Its theoretical background covers the vortex-lattice or panel method depending on user choice which then can be applied to either 2-D airfoil design or 3-D airplane design.

The drag polar of the aircraft's control surfaces could be generated with the help of the XLRF5 software. The standard practice for getting the drag polar for aircraft is to generate the 3-D model in XFLR5 with the help of aircraft building feature and knowing all the important dimensions and angular relations of the aircraft we are building.

The dimensions and the angular relations of the AVISTAR model are defined in Table 3.3. The model information is defined appropriately for XFLR5 to interpret the surfaces for the vortexlattice method used to obtain the solution [37]. 


\begin{tabular}{|c|c|c|c|c|c|c|c|}
\hline \multicolumn{8}{|l|}{ Wing } \\
\hline $\begin{array}{c}\text { LE } x \\
\text { pos }\end{array}$ & $\begin{array}{c}\text { LE } z \\
\text { pos }\end{array}$ & Incidence & $\begin{array}{l}\text { y span } \\
\text { pos }\end{array}$ & Chord & Offset & Dihedral & Airfoil \\
\hline $\begin{array}{l}380.4 \\
\mathrm{~mm}\end{array}$ & $\begin{array}{l}95.5 \\
\mathrm{~mm}\end{array}$ & $3.58 \mathrm{deg}$ & $0 \mathrm{~mm}$ & $\begin{array}{c}237.10 \\
\mathrm{~mm}\end{array}$ & $0 \mathrm{~mm}$ & $0.9 \mathrm{deg}$ & AVISTAR Wing \\
\hline- & - & - & $\begin{array}{c}793.75 \\
\mathrm{~mm}\end{array}$ & $\begin{array}{c}237.10 \\
\mathrm{~mm}\end{array}$ & $0 \mathrm{~mm}$ & - & AVISTAR Wing \\
\hline \multicolumn{8}{|c|}{ Horizontal Stabilizer } \\
\hline $\begin{array}{c}\text { LE } x \\
\text { pos }\end{array}$ & $\begin{array}{c}\text { LE } z \\
\text { pos }\end{array}$ & Incidence & $\begin{array}{c}\text { y span } \\
\text { pos }\end{array}$ & Chord & Offset & Dihedral & Airfoil \\
\hline $\begin{array}{c}1160 \\
\mathrm{~mm}\end{array}$ & $\begin{array}{c}-2.04 \\
\mathrm{~mm}\end{array}$ & $2.36 \mathrm{deg}$ & $0 \mathrm{~mm}$ & $210 \mathrm{~mm}$ & $0 \mathrm{~mm}$ & $0 \mathrm{deg}$ & $\begin{array}{l}\text { AVISTAR } \\
\text { HT_ROOT }\end{array}$ \\
\hline- & - & - & $291 \mathrm{~mm}$ & $110 \mathrm{~mm}$ & $\begin{array}{l}100 \\
\mathrm{~mm}\end{array}$ & - & $\begin{array}{c}\text { AVISTAR } \\
\text { HT_TIP }\end{array}$ \\
\hline \multicolumn{8}{|c|}{ Vertical Stabilizer } \\
\hline $\begin{array}{c}\mathbf{L E x} \\
\operatorname{pos}\end{array}$ & $\begin{array}{c}\text { LE z } \\
\text { pos }\end{array}$ & Incidence & $\begin{array}{c}\text { y span } \\
\text { pos }\end{array}$ & Chord & Offset & Dihedral & Airfoil \\
\hline $\begin{array}{c}1160 \\
\mathrm{~mm}\end{array}$ & $\begin{array}{c}17.96 \\
\mathrm{~mm}\end{array}$ & $2.36 \mathrm{deg}$ & $0 \mathrm{~mm}$ & $273 \mathrm{~mm}$ & $\begin{array}{l}-95 \\
\mathrm{~mm}\end{array}$ & 0 deg & $\begin{array}{l}\text { AVISTAR } \\
\text { VT_ROOT }\end{array}$ \\
\hline- & - & - & $200 \mathrm{~mm}$ & $96 \mathrm{~mm}$ & $\begin{array}{l}133 \\
\mathrm{~mm}\end{array}$ & - & $\begin{array}{c}\text { AVISTAR } \\
\text { VT_TIP }^{\text {ATIP }}\end{array}$ \\
\hline
\end{tabular}

Table 3.3 “AVISTAR” UAV lifting surfaces dimensions [37]

Using the data defined and the airfoil data obtained from the references and the measurements made from the original RC model, XFLR5 input has been generated. In Figure 3.10 airfoil coordinates for all lifting surfaces are plotted. 


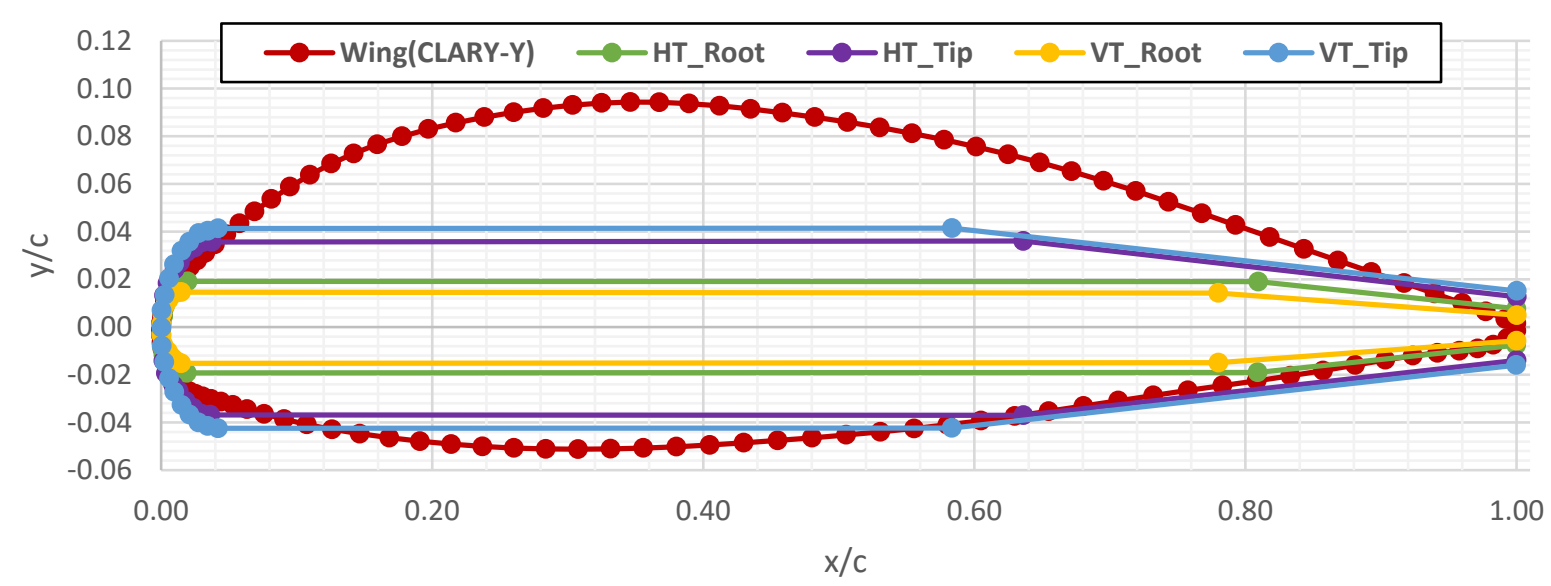

Figure 3.10 AVISTAR airfoils

Next is to use these airfoils in XFLR5 direct airfoil design section with the dimension for wing horizontal tail and vertical tail to create a 3-D model of AVISTAR lifting surfaces in XFLR5. Two-step analysis is run for the generated 3D model; the first one is for fixed-speed and varying angle of attack (Type I), and the second one is fixed angle of attack(lift) but this time varying with speed (Type IV). These drag calculations are calculated on standard atmospheric conditions with viscous effects included. 


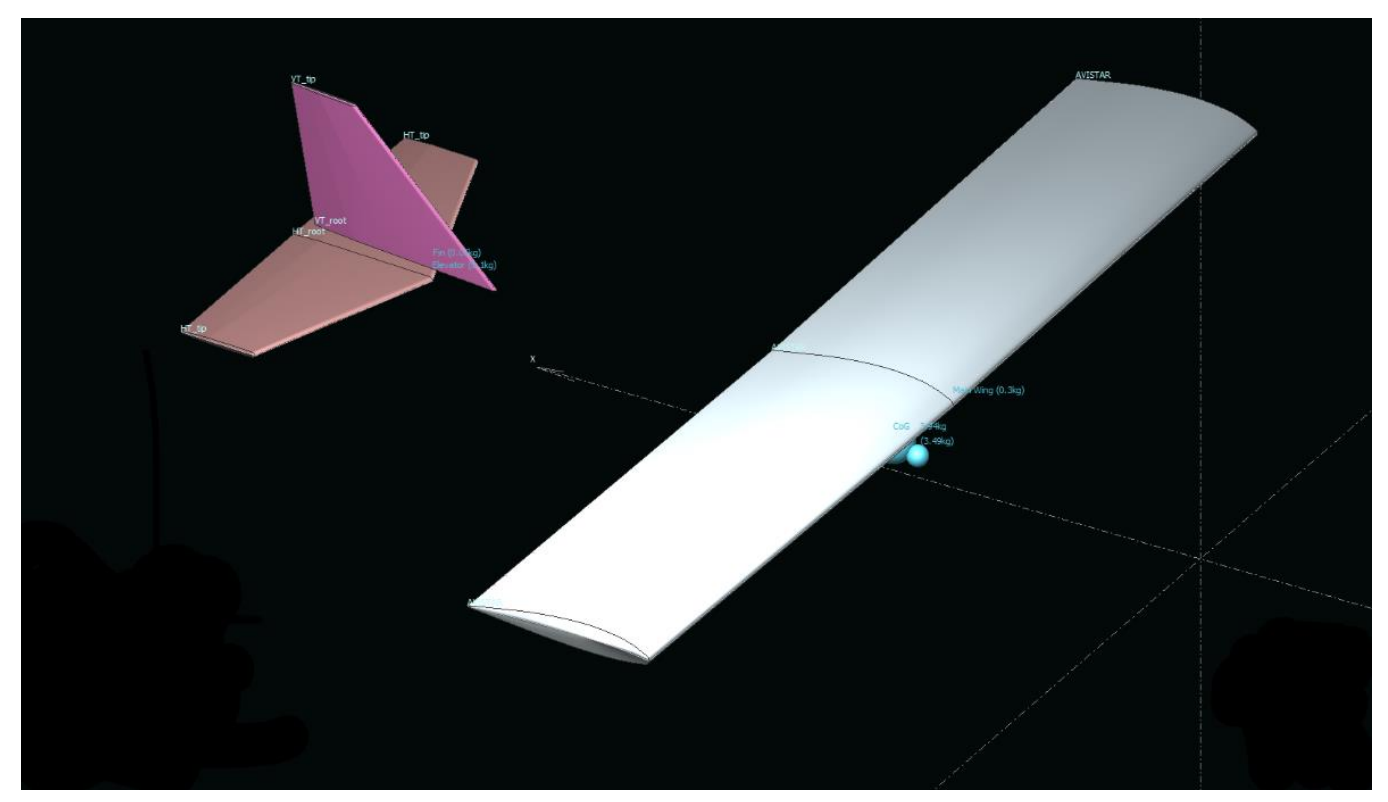

Figure 3.11 XFLR5 Aerodynamics analysis of AVISTAR UAV

In the first analysis lift coefficient is plotted with respect to the angle of attack ranging from -10 to 10 degrees for both take-off and in-flight portions. Take-off includes a ground effect for height of the wing from the ground which is $0.05 m$ entered to XFLR5. 


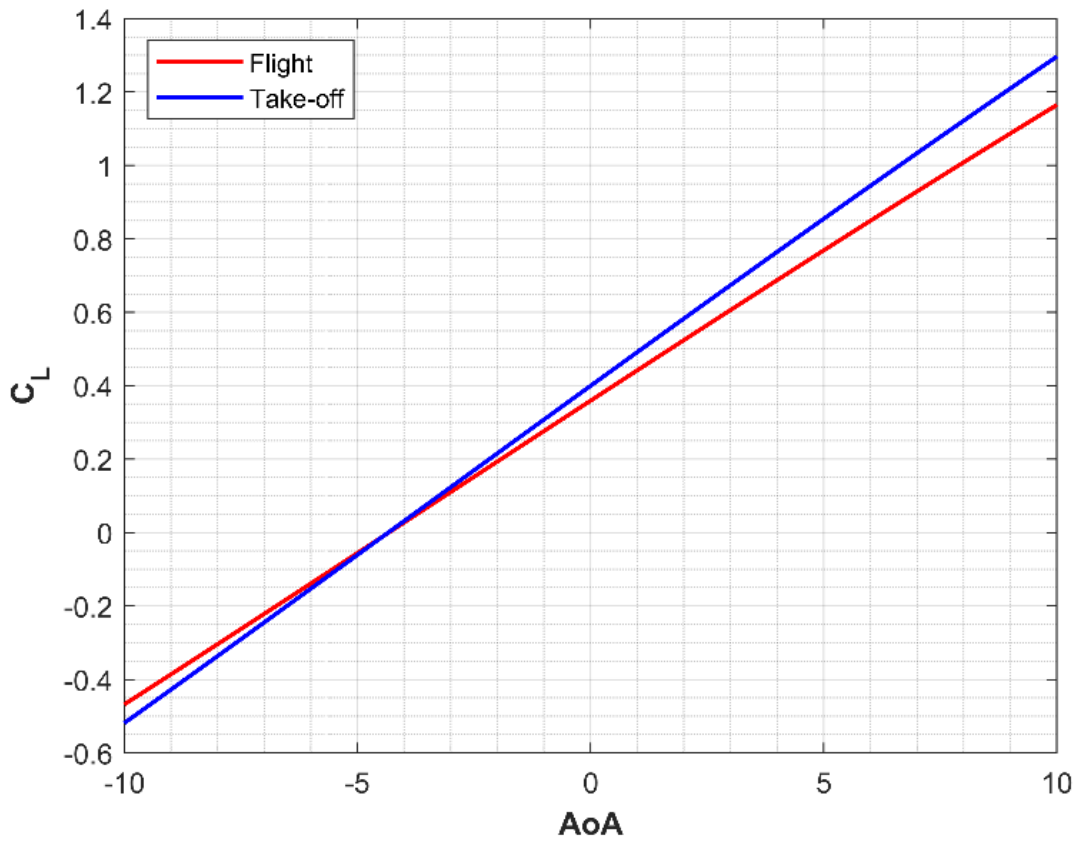

Figure $3.12 C_{L}$ vs AoA 


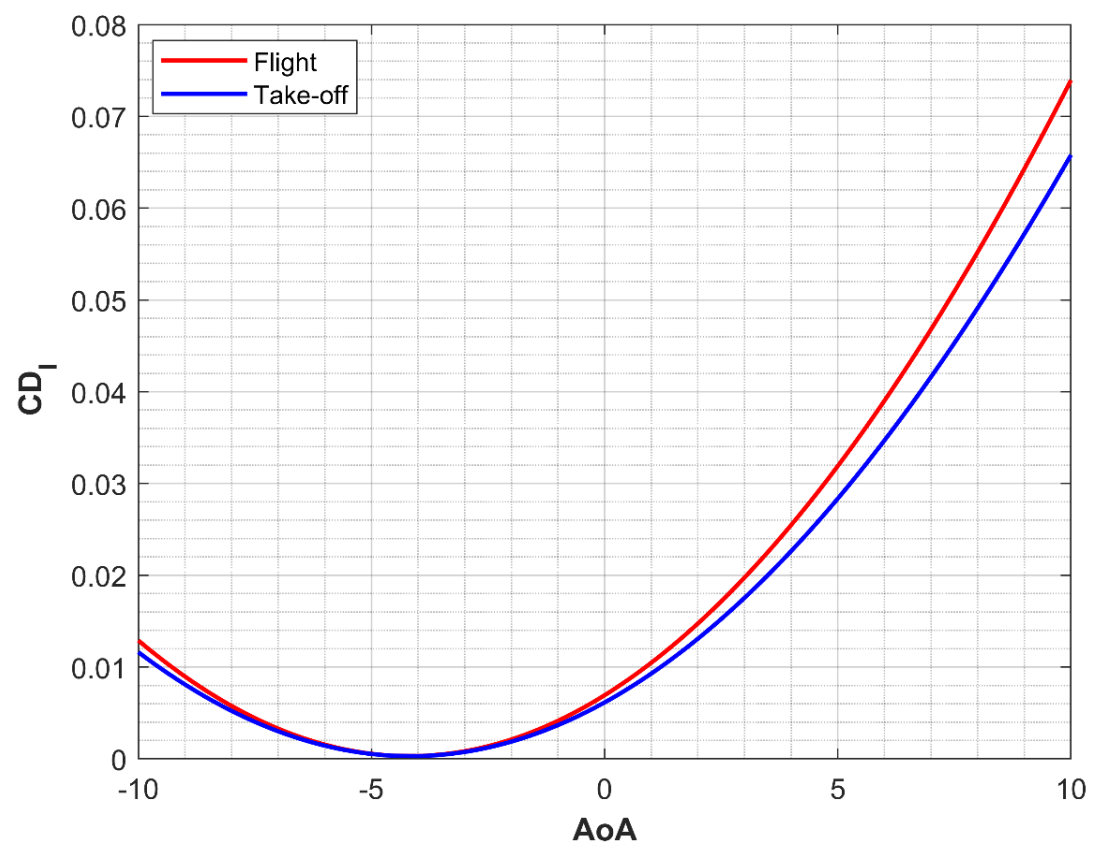

Figure 3.13 $C D_{I}$ vs $A o A$

The contribution of the ground effects is shown in Figure 3.12. The ground effect clearly provides a bit more lift which effectively improves the take-off run. From Figure 3.12 zero lift angle of attack $\alpha_{L=0}$ can be calculated. The graphical solution gives approximately $\alpha_{L=0} \cong$ $-4.33^{\circ}$. Hence, the next analysis, which is Type IV in XLFR5, will be conducted when the angle of attack equals the zero-lift angle of attack. This way the correct viscous drag coefficient is calculated with varying speeds. 


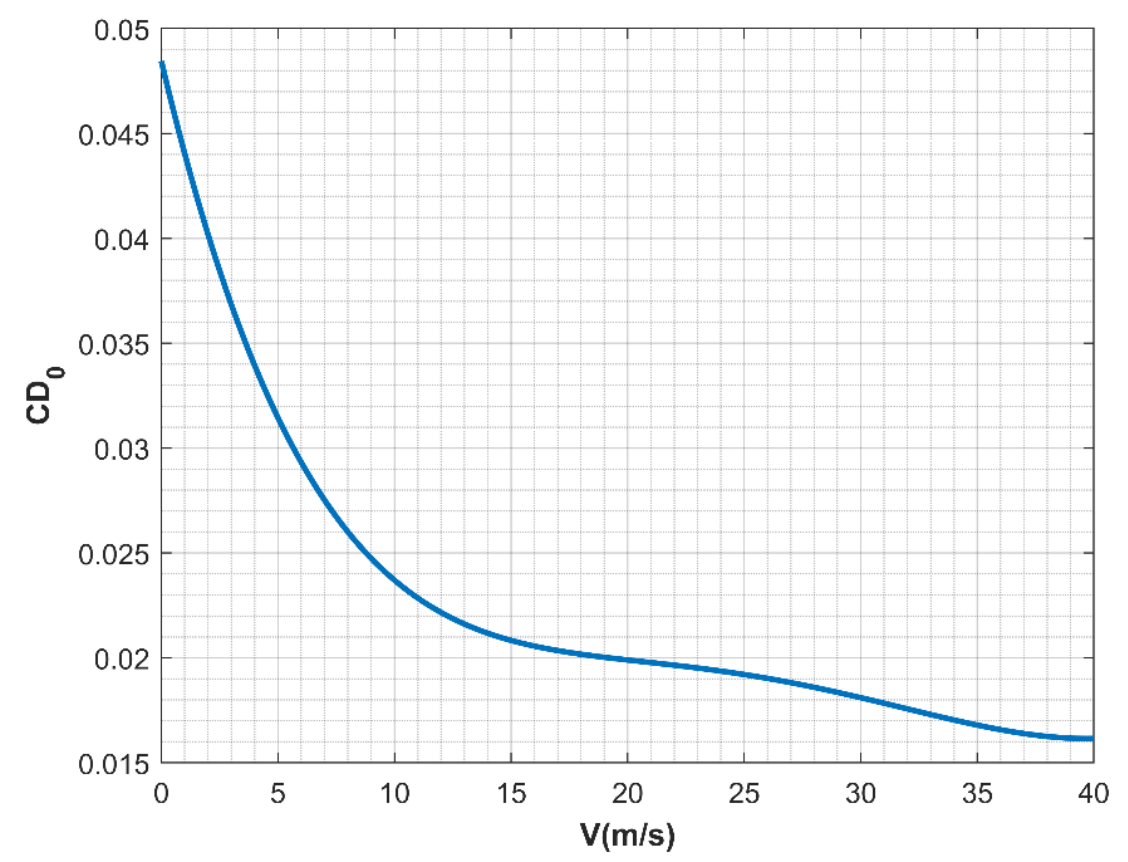

Figure 3.14 $C D_{0}$ vs $V(m / s)$

In order to estimate the overall drag polar of the UAV (i.e. skin friction, form, and interference drag) a component drag buildup model is used. The following models use flat plate calculations of skin friction values modified by the form and interference factor defined in textbooks $[38,39]$ to estimate the drag for each individual component and interaction effect between them. These are called the regression formulas and are obtained from experimental investigations. The following formulations are integrated into the source code which calls on the lookup tables of drag coefficient.

The focal point of the regressions models is to calculate the drag coefficient for the fuselage. XFLR5 could not be utilized for this task since the vortex lattice method has limitations 
for calculating interaction between the wing and fuselage which causes unrealistic flow modelling and pressure gradients at the connection of the wing and fuselage [40].

The Mixed Laminar-Turbulent skin friction coefficient for the fuselage is given as

$$
C_{f}=\frac{0.074}{R e^{0.2}}\left(1-\left(\frac{X_{t r}-X_{0}}{C}\right)\right)^{0.8}
$$

where $X_{t r}$ is the transition location from laminar to turbulent flow, which is selected depending on coverage of the turbulent region [41]. The transition position is estimated with the following formula:

$$
\left(\frac{X_{0}}{C}\right)=36.9 \times\left(\frac{X_{t r}}{C}\right)^{0.625}\left(\frac{1}{R e}\right)^{0.375}
$$

Form factor for the fuselage is given as [42]

$$
F F=2.939-0.7666 f+0.1328 f^{2}-0.01074 f^{3}+3.275 \times 10^{-4} f^{4}
$$

where $f$ is the fineness ratio of the fuselage. It is the length of the fuselage divided by the average diameter of the fuselage;

$$
f=\frac{l_{f}}{d_{f}}
$$

Hence, the total drag is

$$
C d_{f}=\frac{C_{f} \times F F \times S w e t_{f} \times Q_{f}}{S r e f_{f}}
$$

where $\kappa$ is the skin roughness coefficient and $Q_{f}$ is the interference factor of the fuselage, which takes values ranging from 1 to 1.5 depending on the configuration [43]. In the case of the AVISTAR UAV, a value of 1.1 is appropriate according to the fixed high-wing configuration 
defined in the textbooks. Another effect is the area ratio of a wetted area and the reference area. That is defined in Section 5.1, "Aircraft Specifications".

The Reynolds number defined in Eq. (3.55) and Eq. (3.56) is calculated with the standard definition for Reynolds number. However, there is a certain top limit for Reynolds Number for these equations. In order to get a more accurate estimation, the Cutoff Reynolds number is introduced. The Cutoff Reynolds number takes over if the Reynolds number calculated with the standard formula is greater than the following definition [41]. This is defined to account for the surface qualities if they are less than ideal.

$$
R e_{\text {cutoff }}=38.21 \times\left(\frac{l_{f}}{\kappa}\right)^{1.053}
$$

With the condition of

$$
R e=\frac{\rho V l_{f}}{\mu}>R e_{\text {cutoff }} \Rightarrow R e=R e_{\text {cutoff }}
$$

all the relations are established, and the total drag polar of the aircraft can be calculated ranging with the angle of attack and the different wind speeds for the different flight envelopes that can be achieved with the simulation. 


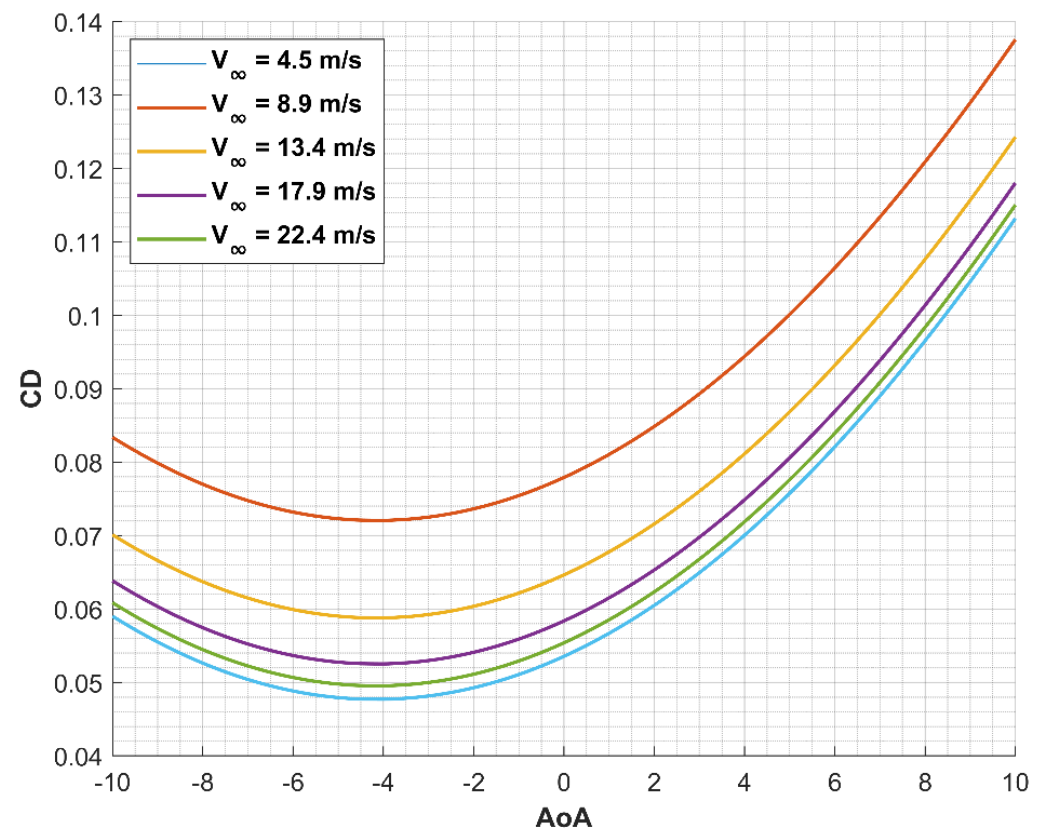

Figure 3.15 CD vs AoA for whole aircraft 


\section{CHAPTER 4}

\section{BATTERY MODELING}

\subsection{CONSTANT POWER DISCHARGE DEVELOPMENT}

Lance Traub's method for estimating discharge for Lithium Battery chemistries builds upon methods established by Peukert [7, 44] which he used for modeling lead-acid batteries. The discharged capacity of the battery at any time instant is obtained by considering the collapsing of a family of different discharge curves obtained experimentally from a battery. In this thesis, the main concern is to measure the state of charge (SOC) which is a close derivative of the discharge curve and can be simply integrated into the system instead of the discharge capacity variable. All the experimentally collected data is analyzed with the appropriately captured time steps and two measured variables: voltage and current. Then, using the "Coulomb Counting Method" SOC is determined as shown in the equation below.

$$
\operatorname{SOC}(t)=\operatorname{SOC}\left(t_{0}\right)-\frac{\int_{t_{0}}^{t} I d t}{C_{0}}
$$

where $I$ denotes the current, and $t_{0}$ is the initial time. This relation integrates the current change between time $t$ and $t_{0}$. Its low computational resource demands that it be very suitable for realtime applications $[45,46]$. As described in reference [7], the constant discharge curves for a PULSE 3300mah 45C Li-Po battery are considered with the datasets of 10A, 20A and 30A discharge rates. The discharge rates with respect to time are then converted to the Voltage vs. SOC curves using "Coulomb Counting”. 
After obtaining the SOC for each time step, a new discharge curve is computed based on the collapsed discharge curves from all available constant current discharge runs using an expression of $I^{n} V$.

The following algorithm as suggested by Lance W. Traub [7] is outlined in sequential steps:

I. Using the available constant current discharge curves for the specific battery, plot $i^{n} V$ is a function of the SOC. The constant " $n$ " denotes the collapse coefficient of the curves, and it can be established by using a non-linear least square minimization. In order to obtain that parameter a minimization function could be written as follows. As an example, the function for three test cases $\left(I_{j}=10 A, 20 A, 30 A\right)$ are the three different discharges for indices $j=$ 1,2,3. The least square criterion is implemented as follows:

$$
S u m=\sum_{j=1}^{3}\left[V_{j} I_{j}^{n}-\overline{V I^{n}}\right]^{2}
$$

The over bar indicates an average for all $j$. A non-linear least square solver lsqnonlin is used to solve this minimization problem with MATLAB. For the battery modeled in the thesis work, $n \cong 0.0392$. This value is close to the 0.05 value of Traub's paper [7] though it can be significantly different in practice due to the different datasets for different batteries.

II. The next step after the value for $\mathrm{n}$ has been determined is to construct a curve fitting function for the collapsed curves. The following quadratic polynomial function is fitted for the new collapsed curves [7]:

$$
V I^{n}(S O C)=\left(a+c S O C+e S O C^{2}\right) /\left(1+b S O C+d S O C^{2}+f S O C^{3}\right)
$$


This form of a fitting function is fitted by using MATLAB's optimization database. Hence, by simply defining the fit type to the above function and using a $f i t(x, y, f t, o p t i o n s)$ where

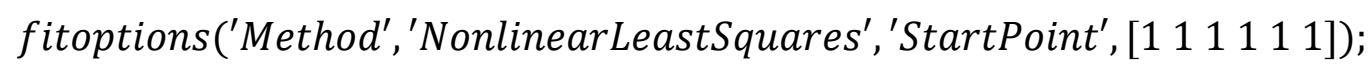

$$
f t=f i t t y p e\left({ }^{\prime}\left(a+c * x+e * x^{\wedge} 2\right) /\left(1+b * x+d * x^{\wedge} 2+f * x^{\wedge} 3\right)^{\prime}\right) ;
$$

Figure 4.1 MATLAB fit options for the Nonlinear Least Squares

Coefficients are provided and \%95 confidence bounds are available:

$$
\begin{array}{|l|}
\hline a=-3.991 \\
\hline b=1.746 \\
c=6.291 \\
d=-3.238 \\
e=-10.27 \\
f=0.4944
\end{array}
$$

Table 4.1 Polynomial fit coefficients 
III. The voltage during the discharge may be found with:

$$
V_{j}=\left(I^{n} V(S O C)_{j-1}\right) / I_{j}^{n}
$$

Index $\mathrm{j}$ in the equation indicates that whether the value for the parameter is taken from the current or the previous time step. Also, knowing the relation $I_{j}=P_{e} / V_{j}$ which is substituted into the Voltage equation established above yields [7]:

$$
V_{j}=\left(\frac{I^{n} V(S O C)_{j-1}}{P_{e}^{n}}\right)^{\frac{1}{1-n}}
$$

where $P_{e}$ is the power required by the battery, and it is obtained directly from the power curves of the propeller and adjusted for the propulsion system efficiency.

$$
P_{e}=P_{r e q} / \eta_{t o t}
$$

where $\eta_{\text {tot }}$ is the efficiency of the propulsion system including propeller, electronic speed controller (ESC) and the motor. This process is later discussed in the subsequent chapter.

IV. The corresponding current at the $j_{t h}$ time step is:

$$
I_{j}=\frac{P_{e}}{V_{j}}
$$

V. The discharged capacity and current SOC is calculated as:

$$
D_{j}=I_{j} \times(\Delta t)+D_{j-1}
$$

where $D$ is the discharged capacity and the SOC relation is shown below [7].

$$
S O C_{j}=\frac{C_{0}-D_{j}}{C_{0}}
$$

where $C_{0}$ is the rated capacity of the battery in $A m p \times$ hours units and the battery selfdischarge effects are neglected for the purposes of this simulation. Hence, the simulation takes place in a fraction of the time so that any static effects are negligible. 
VI. Calculate voltage, current and discharged capacity at the next time step $t$. Make sure the time step is small enough to capture the effects of the transient changes in the voltage and current.

VII. Repeat steps III-V until the SOC reaches the limit or the mission simulation has completed.

The raw data was collected from the experiment. Since some of the data was noisy Leastsquares, spline approximation was applied to the data in order to smooth the results. The resulting plot is shown below.

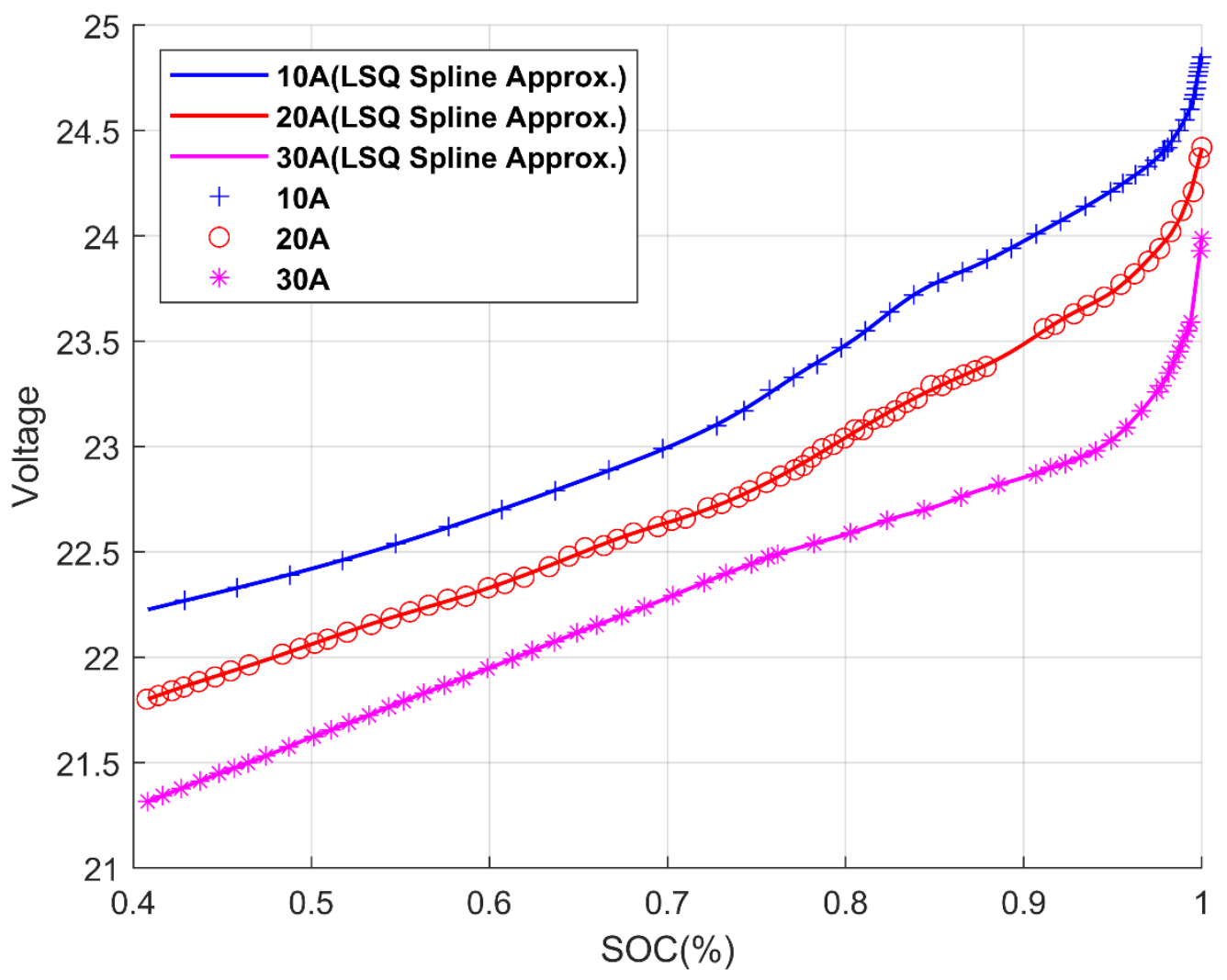

Figure 4.2 Lab battery discharge test data for PULSE 3300mAh 6S 22.2V 45C LiPo Battery 
Correlated discharged curves obtained for the three battery discharge solutions for $n=0.0392$ are shown below in Figure 4.3.

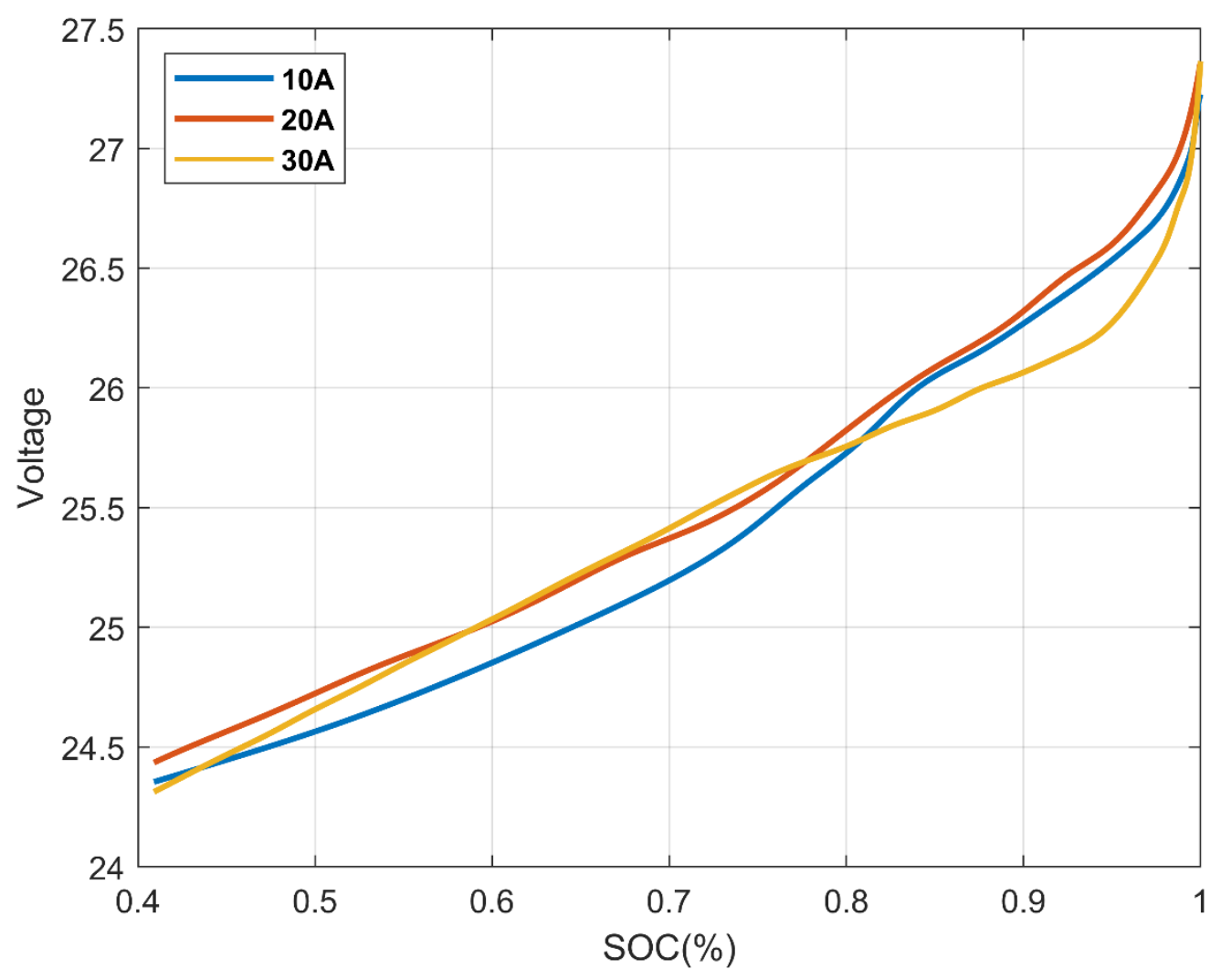

Figure 4.3 Collapsed discharge curves (n=0.0392) for PULSE 3300mAh 6S 22.2V 45C LiPo Battery 


\subsection{SIMULINK BATTERY CIRCUIT MODELING}

\subsubsection{Parameter Estimation Setup}

A SIMULINK model was determined for the validation portion of the battery analysis that was developed in the previous section using the constant power technique. However, this technique is not able to capture the transient voltage drops accurately. Implementation of the Simulink battery model is not for replacing the main estimation model that is developed but to check whether the calculation is performed effectively on the constant or relaxed portion of the power curves during the simulation effort.

The primary analysis done with Simulink utilizes a single RC block, and it is a good estimation for the preliminary search on battery estimation. There is also 2-RC model constructed which is the improved version of the single RC block model. The primary advantages of the 2-RC model are that it can capture the short- and long-time constants with the consequent two RC networks and the internal series resistor captures the immediate voltage drop of the battery system. However, the main validation model was selected to be the single RC circuitry. This decision was based on the realization that a single RC model is simpler to implement and gives a sufficiently accurate solution for linear current changes and with the operating current values considered for the aircraft, it most likely will perform without any significant divergence of the solution from the improved 2-RC model which is a significant increase in computational memory. Figure 4.4 demonstrates the model constructed in the Simulink ${ }^{\mathrm{TM}}$ environment using the Simscape ${ }^{\mathrm{TM}}$ blocks and tools. 


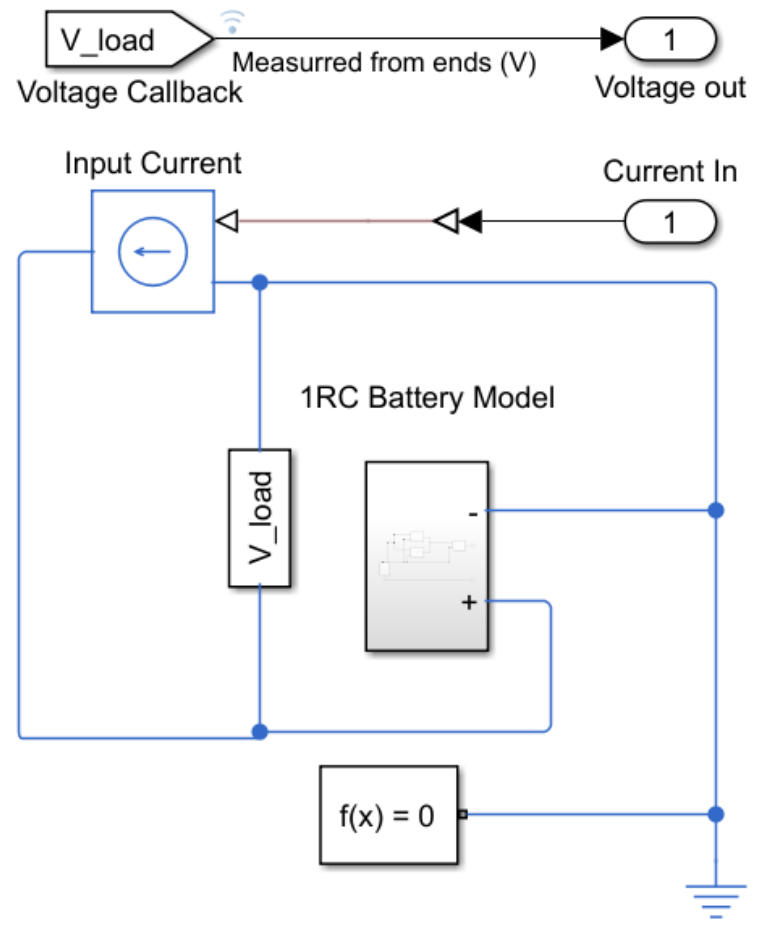

Figure 4.4 Simulink ${ }^{T M}$ library Model for single RC circuit battery modeling system 


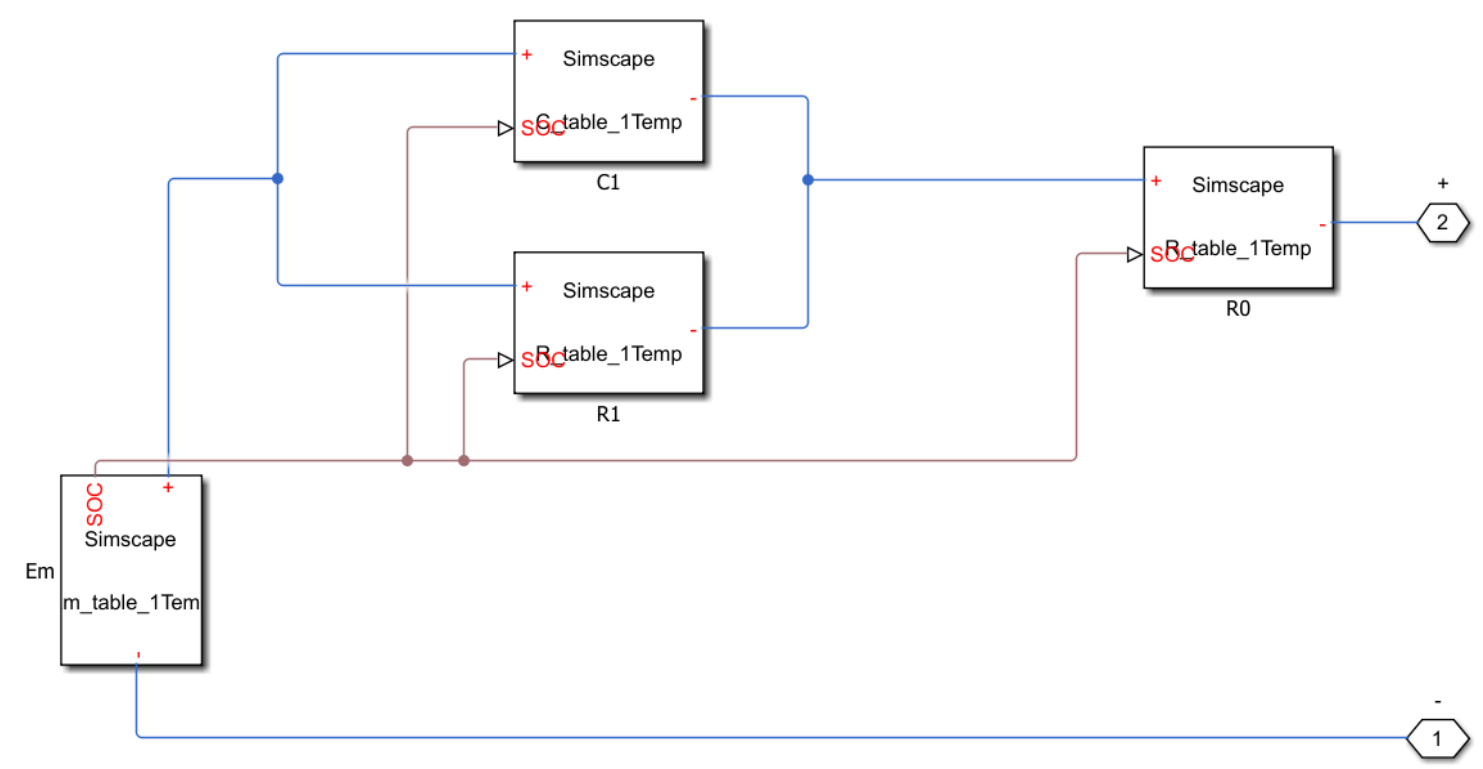

Figure 4.5 Simple single RC ECM circuit in Simulink ${ }^{T M}$

An experimental pulse discharge test was conducted in the lab environment. A Pulse LiPo, 3300Mah size battery was tested to provide real data for the Simscape ${ }^{\mathrm{TM}}$ model in the Simulink $^{\mathrm{TM}}$ environment. The discharging simulation performed with roughly 0.1 change in the battery system capacity at each current load. Current load for each discharge pulse is selected as $20 A(\sim 6 C)$. An estimated "60" seconds of load calculated is necessary to provide one-tenth of a drop in SOC from examples in the literature of pulse battery testing $[44,14,15,19]$.

Due to the nature of the experimental test there are some errors regarding the adjustment of current draw, which was done manually. The aim is to obtain as close as possible to a constant pulse discharge which can be introduced to the Simulink system to get a generic estimation 
solution for the sample battery. After each current load, enough relaxation was provided to the system in order for the voltage to stabilize to a steady OCV value.

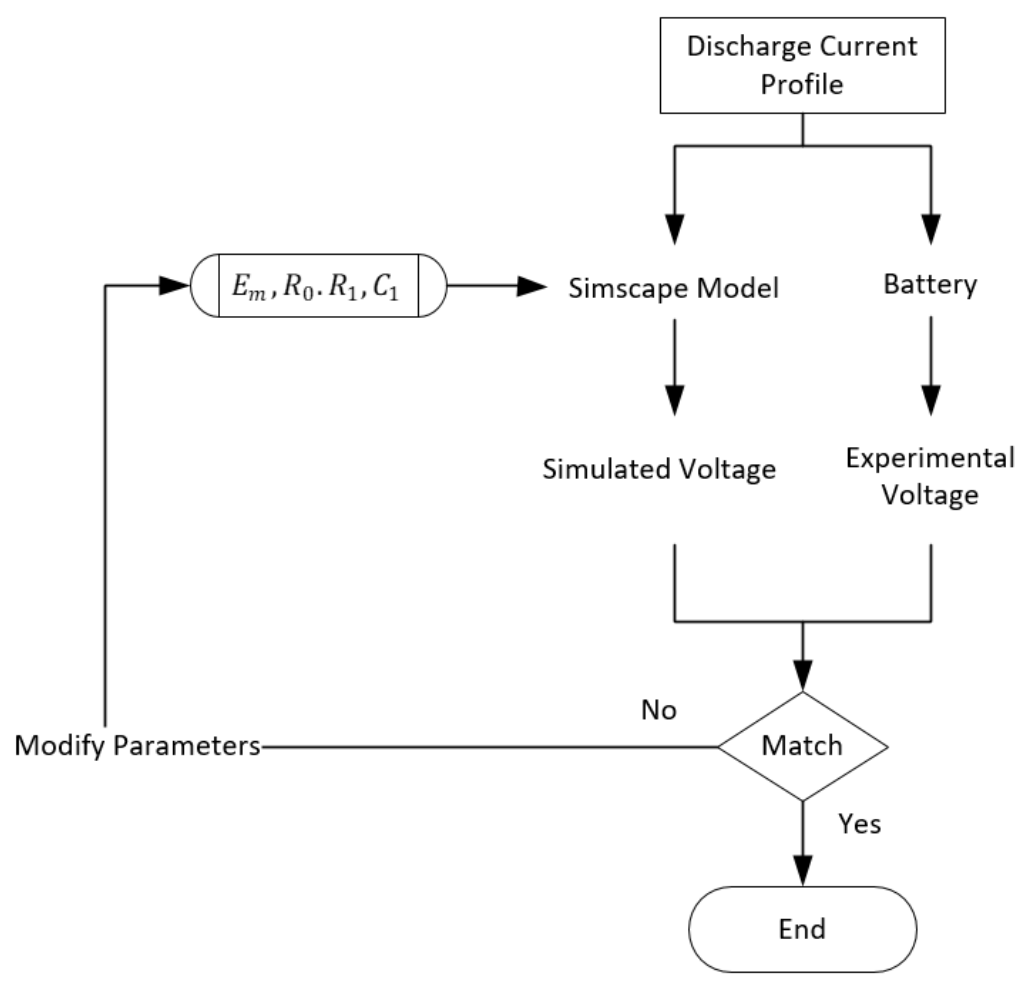

Figure 4.6 Simulink ${ }^{T M}$ parameter estimation flowchart logic

The discharging current was measured with an ETEKCITY MSR-U1000 multi-meter. It provides enough accuracy for the test system for the battery $\pm(0.5 \%+1) @ 4-400 \mathrm{~V}$ is the accuracy provided by the manufacturer manual for DC voltage measuring. 


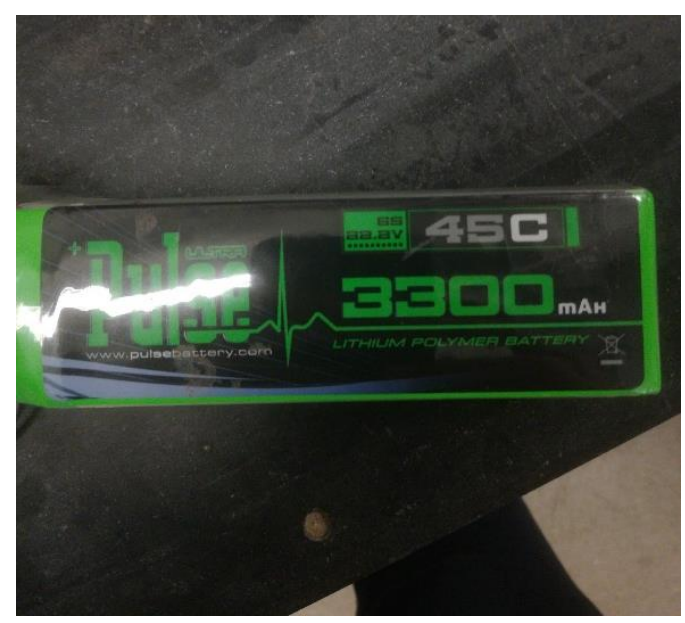

a)

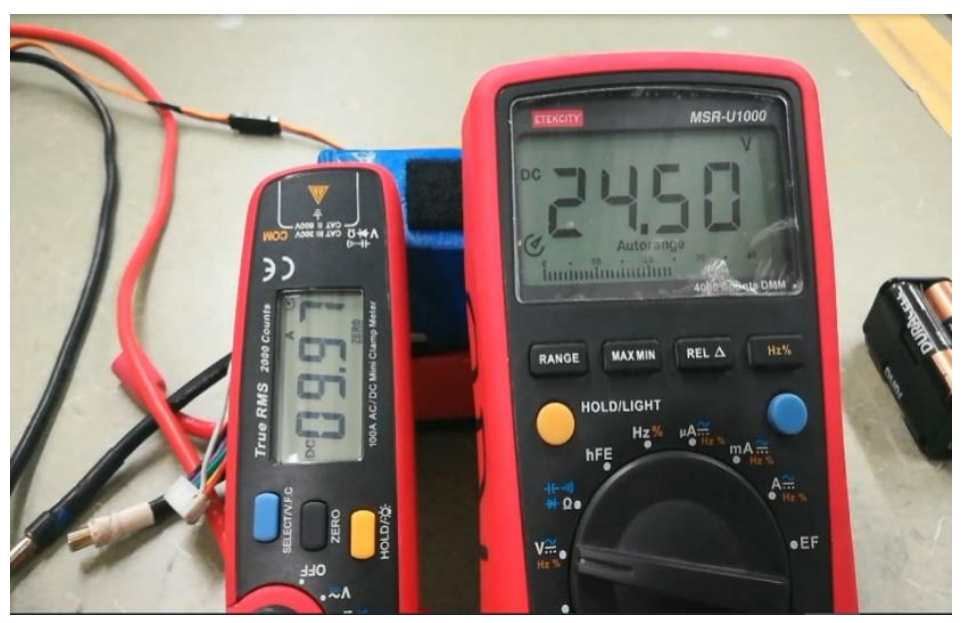

b)

Figure 4.7 a) PULSE 3300mAh LiPo Battery b) ETEKCITY MSR-U1000 \& UNI-T UT210E

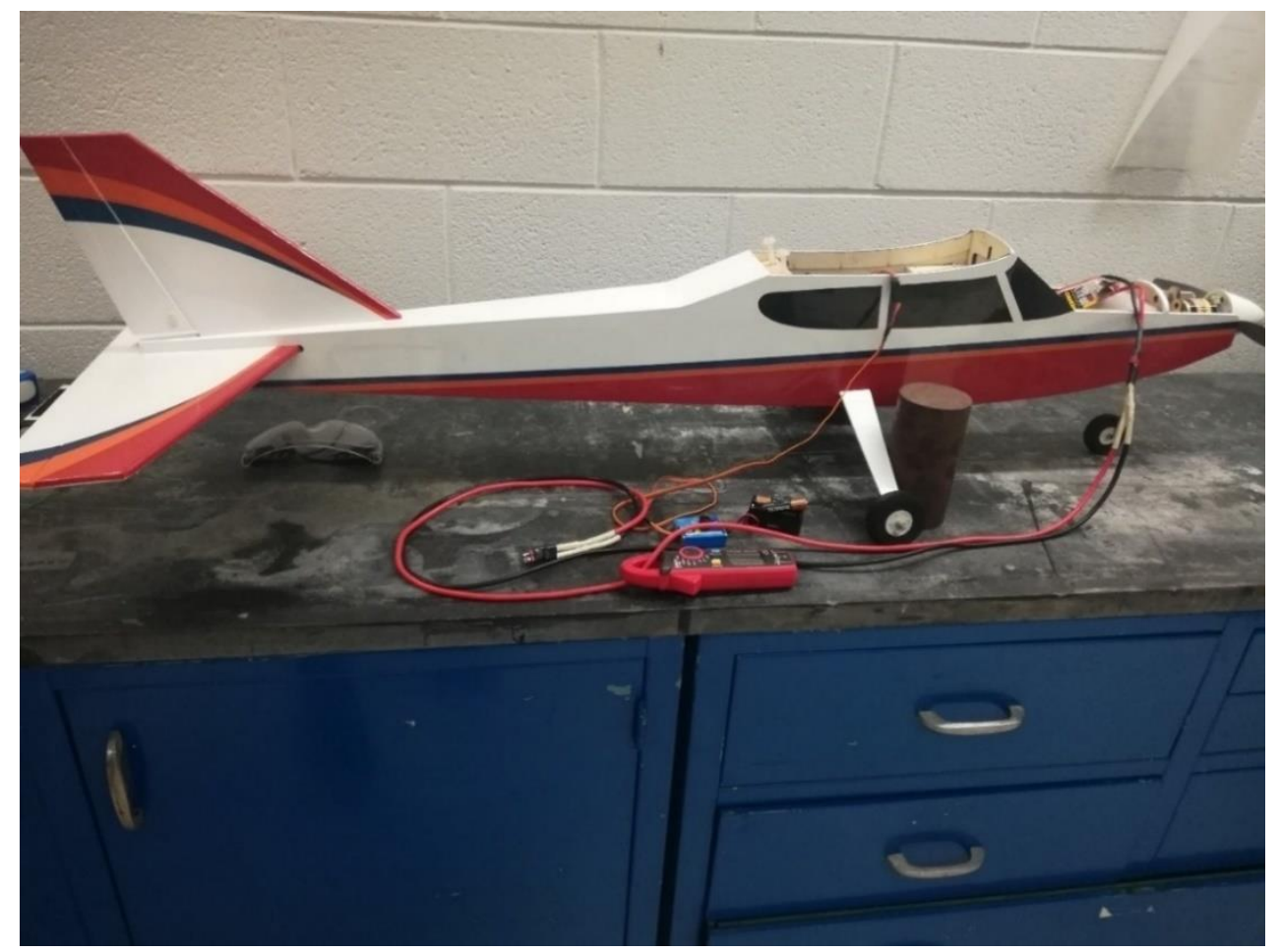

Figure 4.8 AVISTAR RC plane fixed on the lab table for current discharge experiment 
Current measurement was facilitated through a Uni-t Ut210e Digital Clamp Meter. It can measure amperes up to $100 \mathrm{~A}$ and provides a decent estimation for this experimental setup. It provides a 10mA resolution for DC 20A measurement. In the nominal lab environment, following the pulse discharge test, results were logged manually, and the input file was created in MATLAB with correct formatting used in the Simulink ${ }^{\mathrm{TM}}$ parameter estimation module. Simulation is performed with the standard initial values and using the gradient descent method with the SQP (Sequential Quadratic Programming) algorithm. The solution converged after 69 iterations with the tolerance of $1 e-4$. The function minimized to the value of 0.0014 . There are the solutions for the estimation process. 

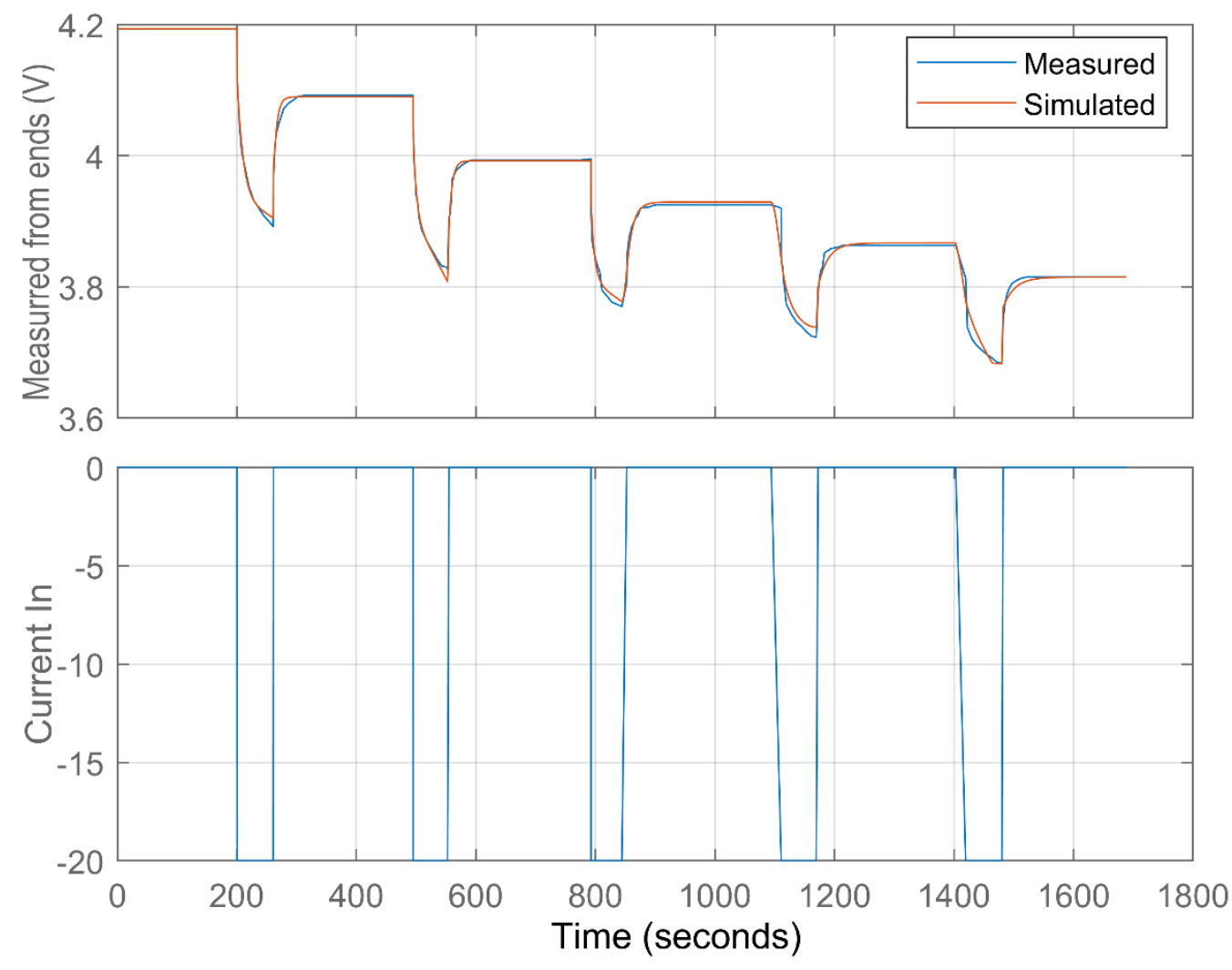

Figure 4.9 Simulation Result for single RC circuit battery system in Simulink ${ }^{T M}$

Negative current signifies the discharge in the experimental method. After the parameter estimation process, battery model parameters were obtained as described in Section 2.2. These coefficients are a function of SOC. Thus, the following look-up tables were generated and are plotted below yielding the solution of the simulation. 


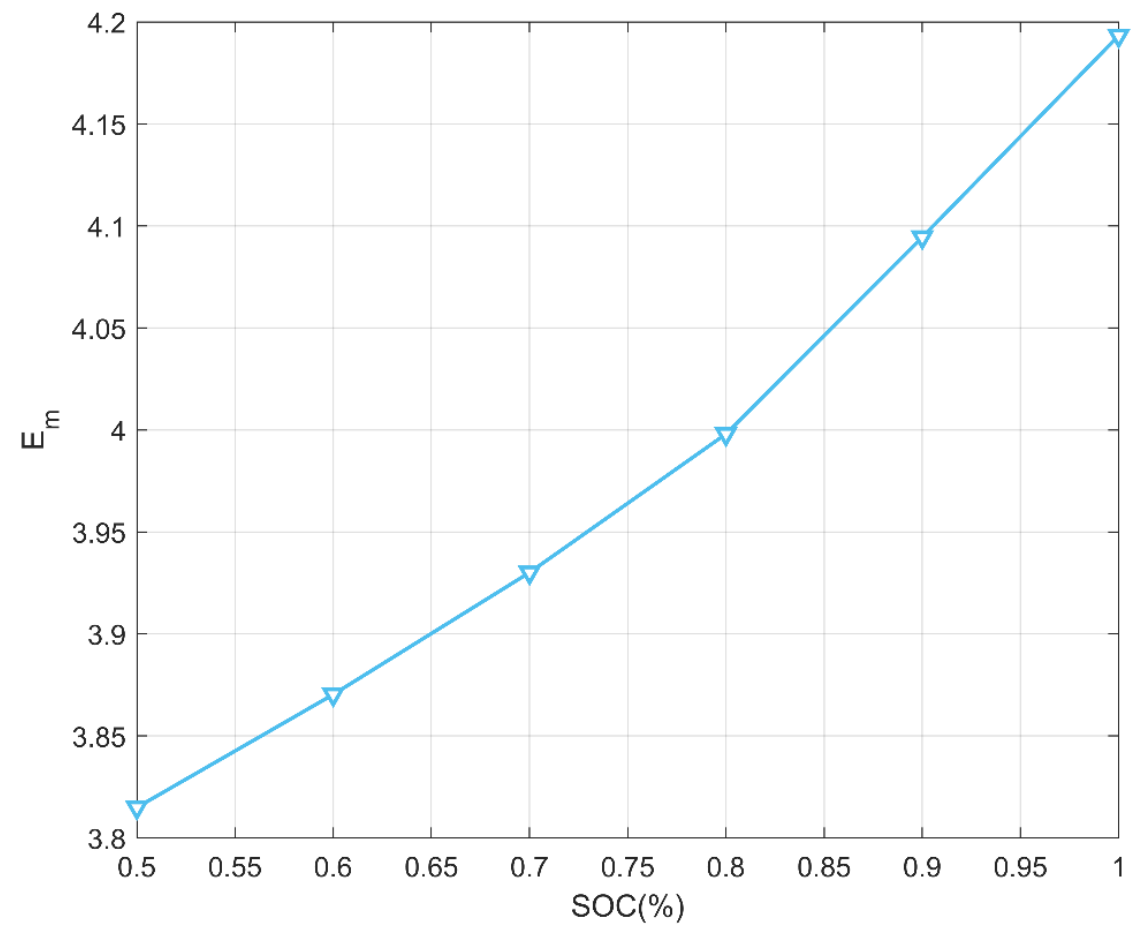

Figure $4.10 E_{m}$ vs SOC plot 


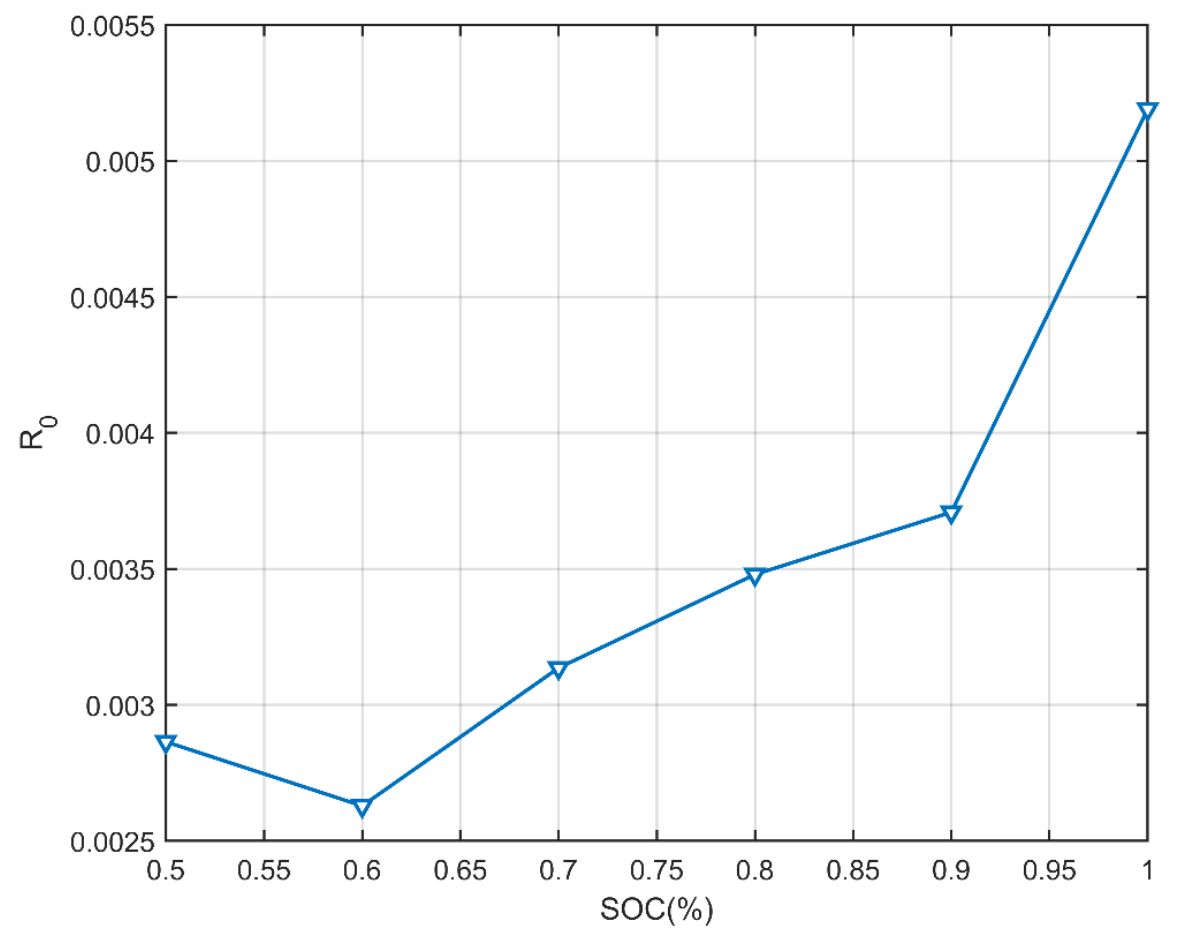

Figure 4.11 $R_{0} v s$ SOC plot 


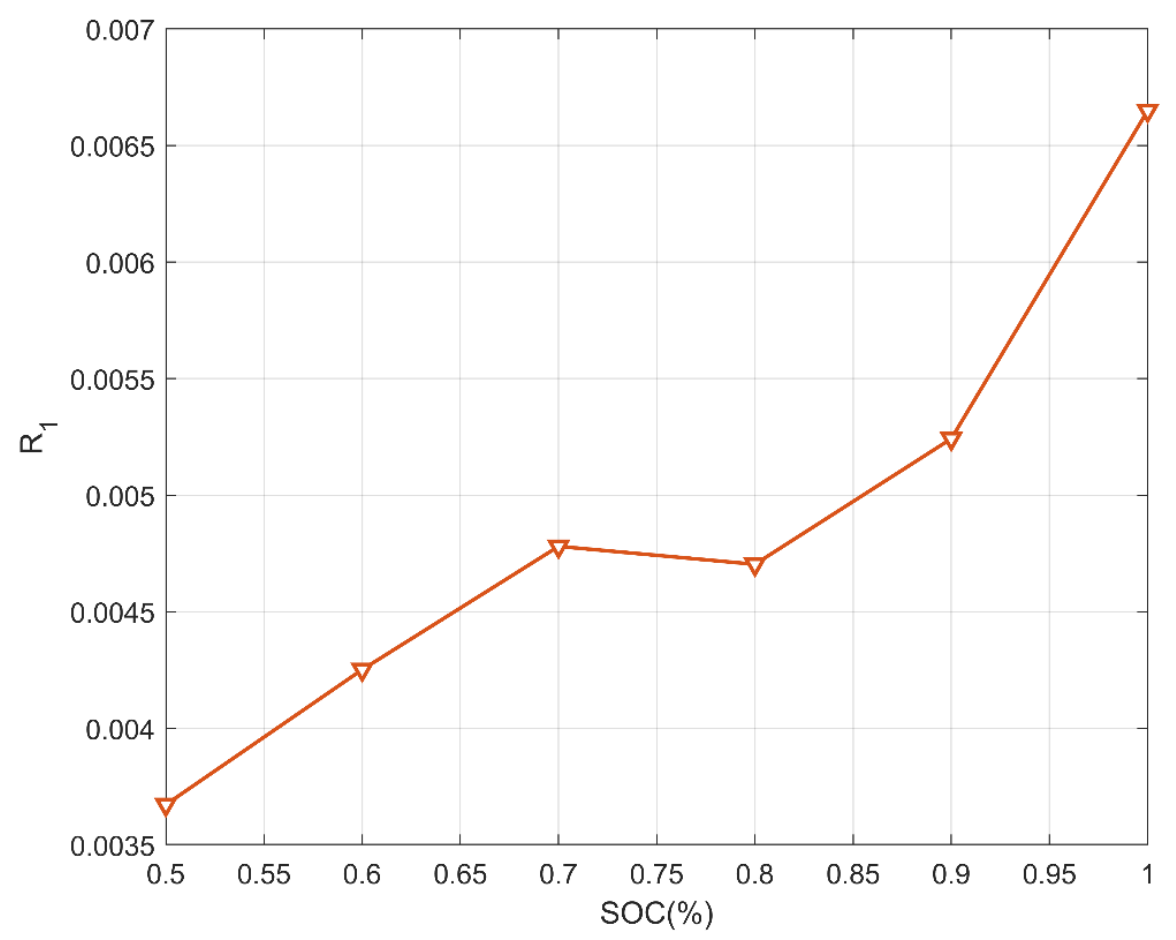

Figure 4.12 $R_{1}$ vs SOC plot 


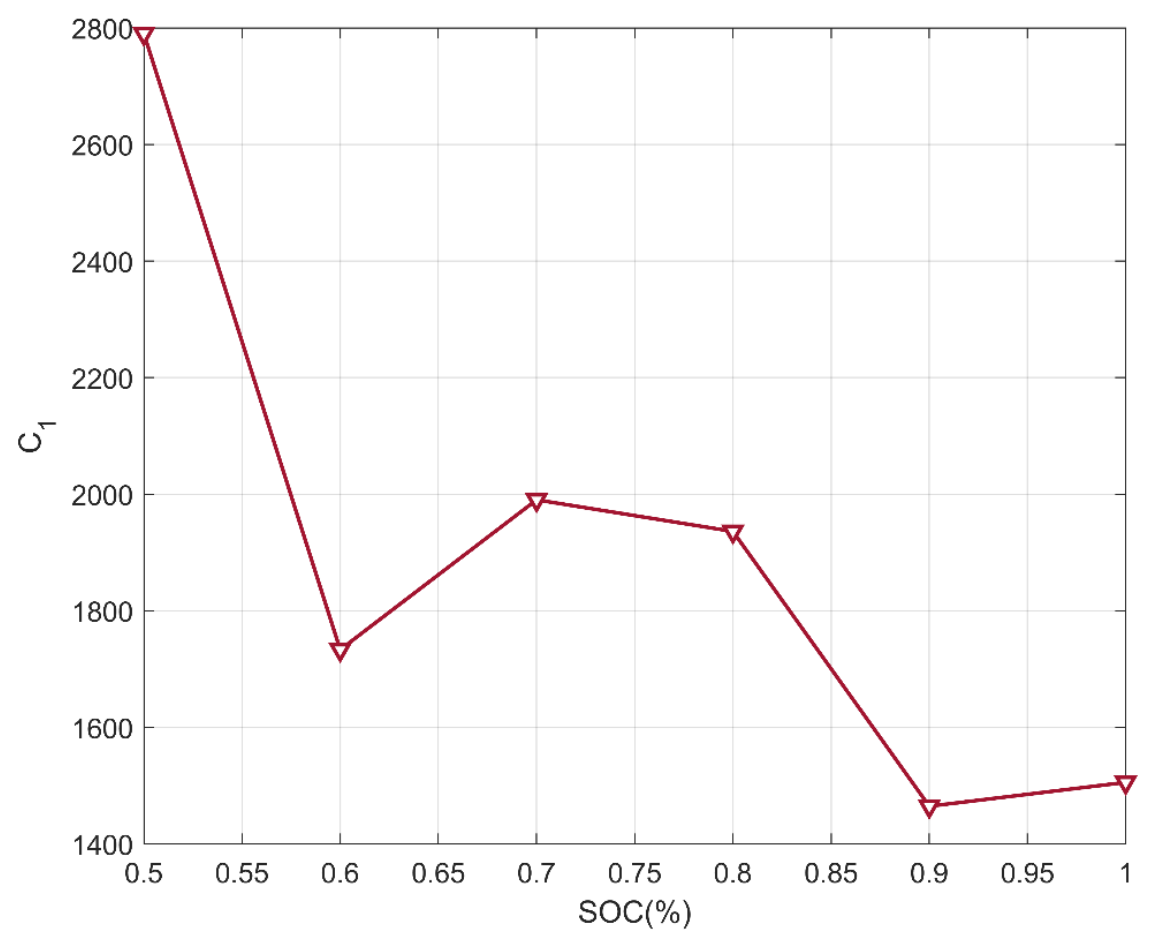

Figure $4.13 C_{1}$ vs SOC plot

These are the coefficients that will create the ECM in the Simulink ${ }^{\mathrm{TM}}$ environment. The planning for the validation procedure follows the listed algorithm below: 
1: $\quad$ FUNCTION Simulink Model

2: $\quad$ INPUT: Starting time; tstart

3: $\quad$ End time; tend

4: $\quad$ Previous SOC value; SOCold

5: $\quad$ Current drawn from the battery at new time step; Amp

6: OUTPUT: New SOC; SOC

7: $\quad$ New terminal voltage for single cell; Vterminal

8: $\quad$ Define the look-up table values from Figure 4.10-Figure 4.13

9: $\quad$ Define Capacity of the battery in $A m p \times$ hours

10: $\quad$ Qe_init $=(1$-SOCold $) *$ Crtd; \% charge deficit

11: Load Simulink system (no gui)

12: $\quad$ Set parameters of the system

13: $\quad$ CALL Run Simulink simulation module from tstart:tend

14: Assign the solutions; SOC,Vterminal

15: END FUNCTION

Algorithm 4.1 Simulink Model pseudo code 


\section{CHAPTER 5}

\section{ELECTRIC UAV SIMULATION OVERVIEW}

The simulation code was written using MATLAB. The main algorithm for the simulation is divided into two parts: a take-off subroutine and a main flight subroutine. Take-off is simulated separately knowing that the equations of motion for runway acceleration are a modified version of the velocity derivative calculated from the primary 5-DOF dynamics equations. These two primary analysis modules are required for a complete simulation of the UAV in 3-D space. The establishment of these modules is critical to perform a conceptual-level sizing and Multidisciplinary design optimization of the particular aircraft type in the environment that is tested [47]. A high-level flow diagram is given in Figure $\mathbf{5 . 1}$ showing the algorithm logic for the simulation. 


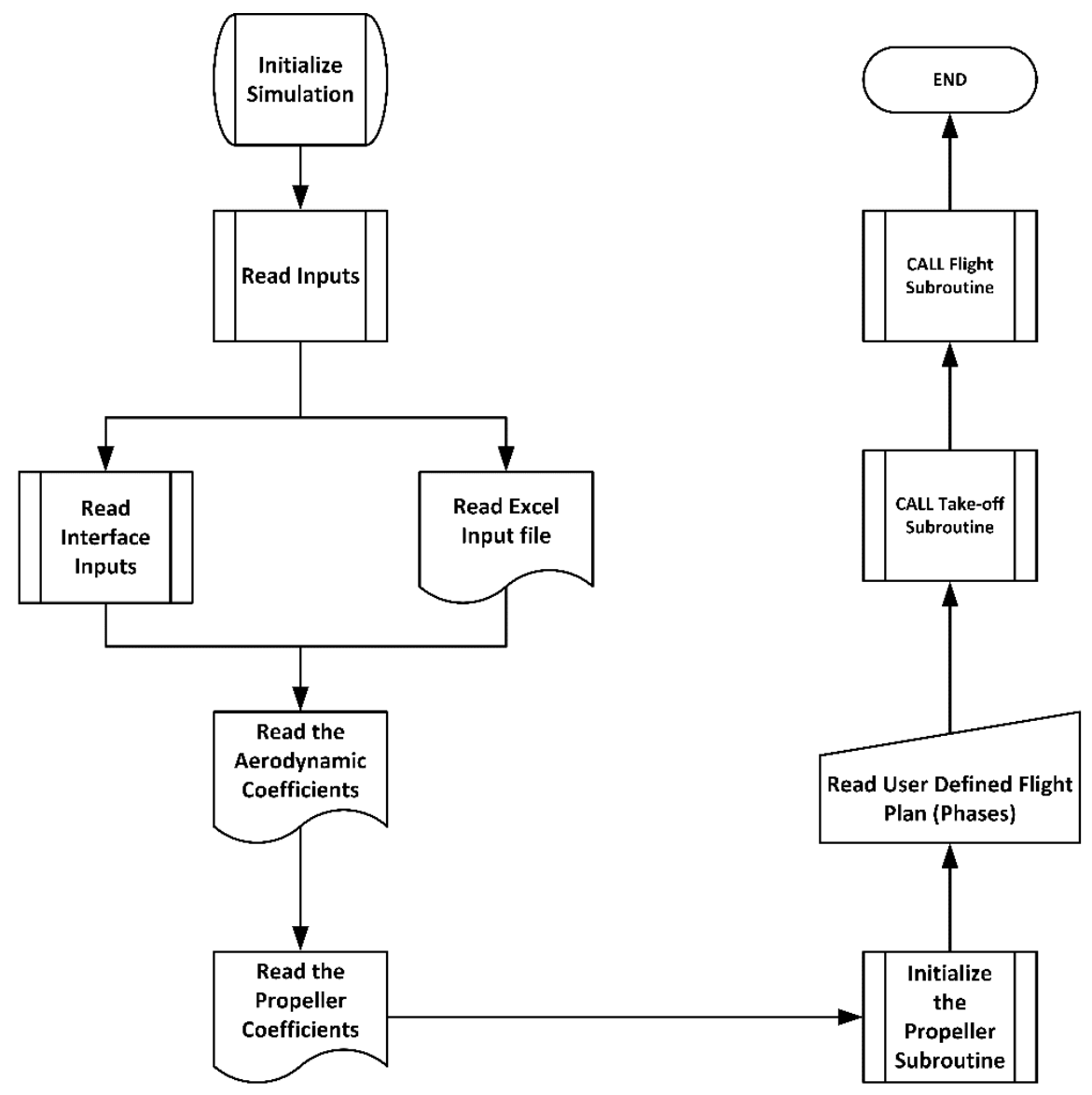

Figure 5.1 flowchart for overall flight simulation script

This simulation chart was produced with the consideration of the order of the sub-systems being generated as a sequence of functions that are being called out by the main subroutines. Simulation is initiated by reading the inputs from the interface or from the excel files that are located in the directory of MATLAB. The main excel file called Inputfile_PFTool consists of four main titles for the input: General-Dimensions, Battery, Simulation, and Configuration. These are defined in Section 5.3. 


\subsection{AIRCRAFT SPECIFICATIONS}

The simulated aircraft AVISTAR UAV is a typical advanced trainer RC model. It has been developed by the manufacturer Great Planes as the Avistar Elite [48]. At the time of this writing it is becoming available in the ODU UAV lab and will serve as a great test bed for the simulation code to run for verification procedures since the real UAV is available to fly. Physical specifications of the aircraft are given in Table 5.1.

\begin{tabular}{|c|c|c|c|}
\hline Geometric Properties & WING & HORIZONTAL TAIL & VERTICAL TAIL \\
\hline Chord $($ Root $)=$ & $0.273 \mathrm{~m}$ & $0.21 \mathrm{~m}$ & $0.273 \mathrm{~m}$ \\
\hline Chord $($ Tip $)=$ & $0.273 \mathrm{~m}$ & $0.11 \mathrm{~m}$ & $0.096 \mathrm{~m}$ \\
\hline Span $=$ & $1.587 \mathrm{~m}$ & $0.582 \mathrm{~m}$ & $0.4 \mathrm{~m}$ \\
\hline Reference Area = & $0.433 \mathrm{~m}^{2}$ & $0.0931 \mathrm{~m}^{2}$ & $0.037 \mathrm{~m}^{2}$ \\
\hline Aspect Ratio = & 5.813 & 3.64 & 2.17 \\
\hline Taper Ratio = & 1 & 1.91 & 2.84 \\
\hline Twist Angle = & $0^{\circ}$ & $0^{\circ}$ & $0^{\circ}$ \\
\hline Incidence Angle = & $3.58^{\circ}$ & $2.36^{\circ}$ & $2.36^{\circ}$ \\
\hline Dihedral = & $0.9^{\circ}$ & $0^{\circ}$ & $0^{\circ}$ \\
\hline Incidence Angle = & $3.58^{\circ}$ & $2.36^{\circ}$ & $2.36^{\circ}$ \\
\hline & \multicolumn{3}{|c|}{ FUSELAGE } \\
\hline Length $=$ & \multicolumn{3}{|c|}{$1.2954 \mathrm{~m}$} \\
\hline Average Diameter $=$ & \multicolumn{3}{|c|}{$0.127 \mathrm{~m}$} \\
\hline Reference Area = & \multicolumn{3}{|c|}{$0.11516106 \mathrm{~m}^{2}$} \\
\hline Wet Area = & \multicolumn{3}{|c|}{$0.6 \mathrm{~m}^{2}$} \\
\hline & \multicolumn{3}{|c|}{ LANDING GEAR } \\
\hline Number of Tires = & \multicolumn{3}{|c|}{3} \\
\hline Tire Width $=$ & \multicolumn{3}{|c|}{$0.01 \mathrm{~m}$} \\
\hline Diameter $=$ & \multicolumn{3}{|c|}{$0.03 \mathrm{~m}$} \\
\hline
\end{tabular}

Table 5.1 AVISTAR Specifications 
Airfoil details are given in Section 3.4.2 for each of the flight surfaces of the Avistar UAV. The tail section of the aircraft has mostly flat-plate shaped airfoils and the wing airfoil is a semisymmetrical Low-Reynolds number airfoil [49]. As described next, propulsion and the electrical systems used in the Avistar are selected according to compatibility. The additional required components that are part of the RC plane and modeled in the simulation are the Motor, Propeller, and ESC used in the aircraft while testing. They are shown in Figure 5.2-Figure 5.4.

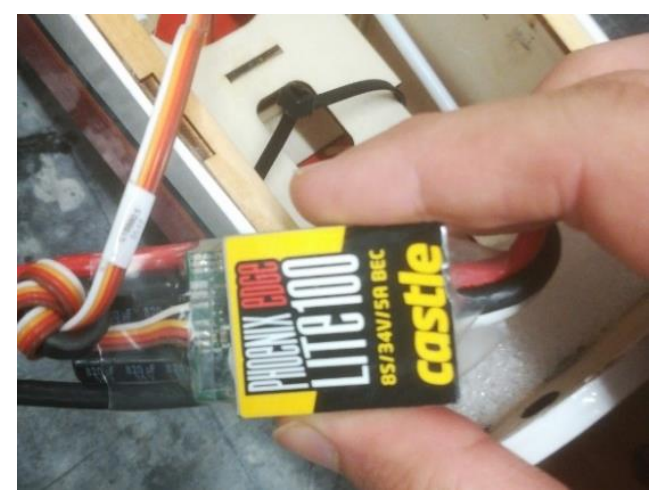

a)

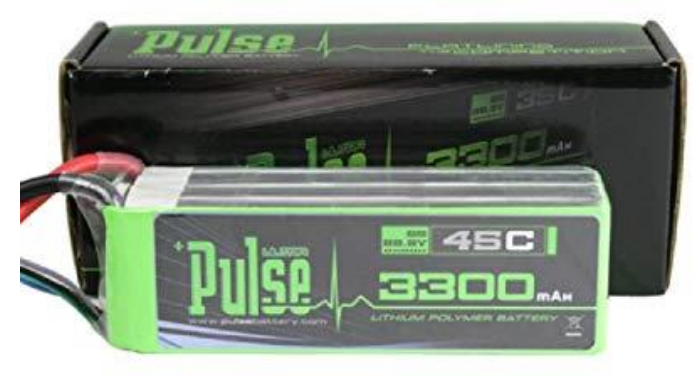

b)

Figure 5.2 a) Phoenix Edge Lite 100 Amp ESC b) Pulse 3300 mAh 45C 6S Li-Po Battery

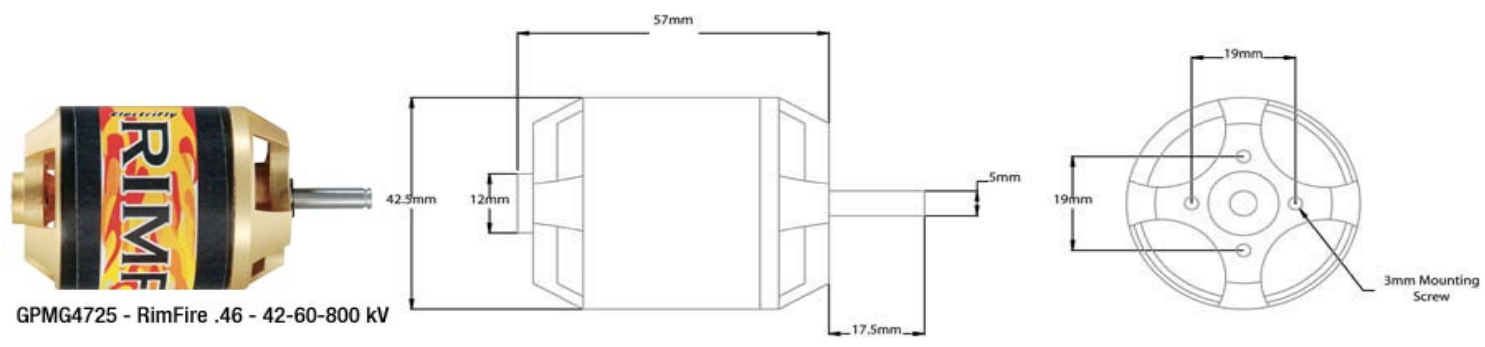

Figure 5.3 Rimfire .46 Brushless Motor dimensions [50] 


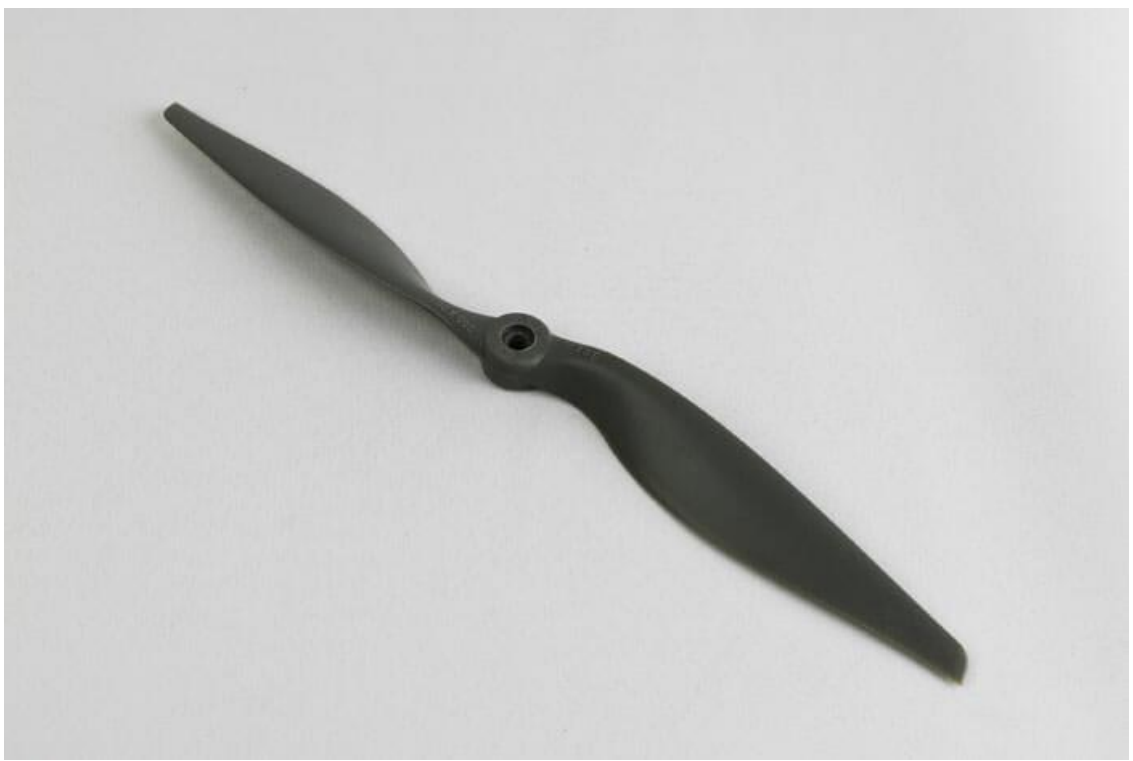

Figure 5.4 APC 12x6E propeller [51] 


\subsection{FLIGHT MISSION FLOW DIAGRAM}

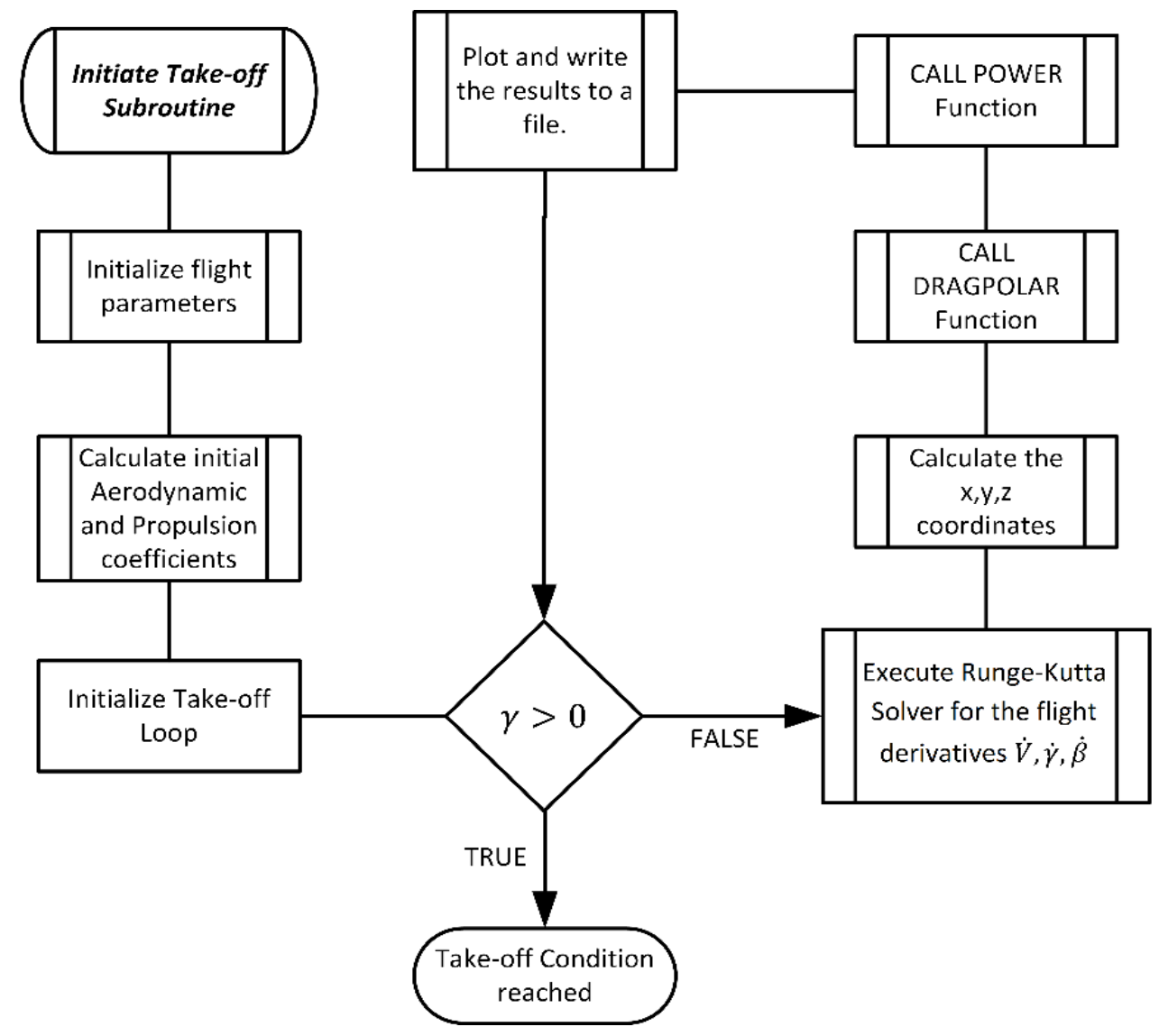

Figure 5.5 Take-off subroutine flowchart

The simulation code initiates with the take-off calculation module. In this part, the take-off run is calculated. Globally defined parameters are read in here that are appropriate for the take-off calculation. These are mainly flight parameters like initial attitude of the aircraft such as heading, pitch angle, angle of attack, flight path angle and the position of the aircraft located in the inertial frame. 
After setting initial conditions, given parameters for the aircraft are set as appropriate aerodynamics coefficients, and the propeller parameters are then calculated in their individual functions and sent to the take-off module.

The main loop is initialized after the inputs are sufficient. In the main loop, Runge-Kutta integration commences with the given thrust data set from the RPM value of the APC $12 \times 6 \mathrm{E}$ propeller. In take-off mode, a coordinated take-off run is assumed with no wind or other disturbances present in the simulation. Only the velocity derivative is being calculated while heading is set to the direction of the artificial runway. In the case of a flight path angle, normally it would give values of negative at slow speeds where lift is smaller than the weight. This will be limited to " 0 " in the simulation since there is no possibility that aircraft move towards the earth from the ground.

The main take-off condition is then defined for the take-off. Take-off condition occurs when the EOM for the flight path angle is greater than " 0 " which means the aircraft finally lifted off from the ground as defined in Eq. (5.1). The speed at which the flight path angle is greater than zero at the first instance is given as the take-off speed. The take-off distance is the distance between initial location of the aircraft and the location where the aircraft lifted.

$$
\begin{gathered}
\gamma_{t_{i}}>0 \\
S_{G}=\int_{0}^{t_{i}} V \cdot d t
\end{gathered}
$$

Eq. (5.2) is the take-off distance in integral form while $i$ is the step number and $t$ is the total time. In the validation portion of the investigation there will be a comparison of how accurate the takeoff calculations are correlated with the empirical methods used in the aircraft design books. 


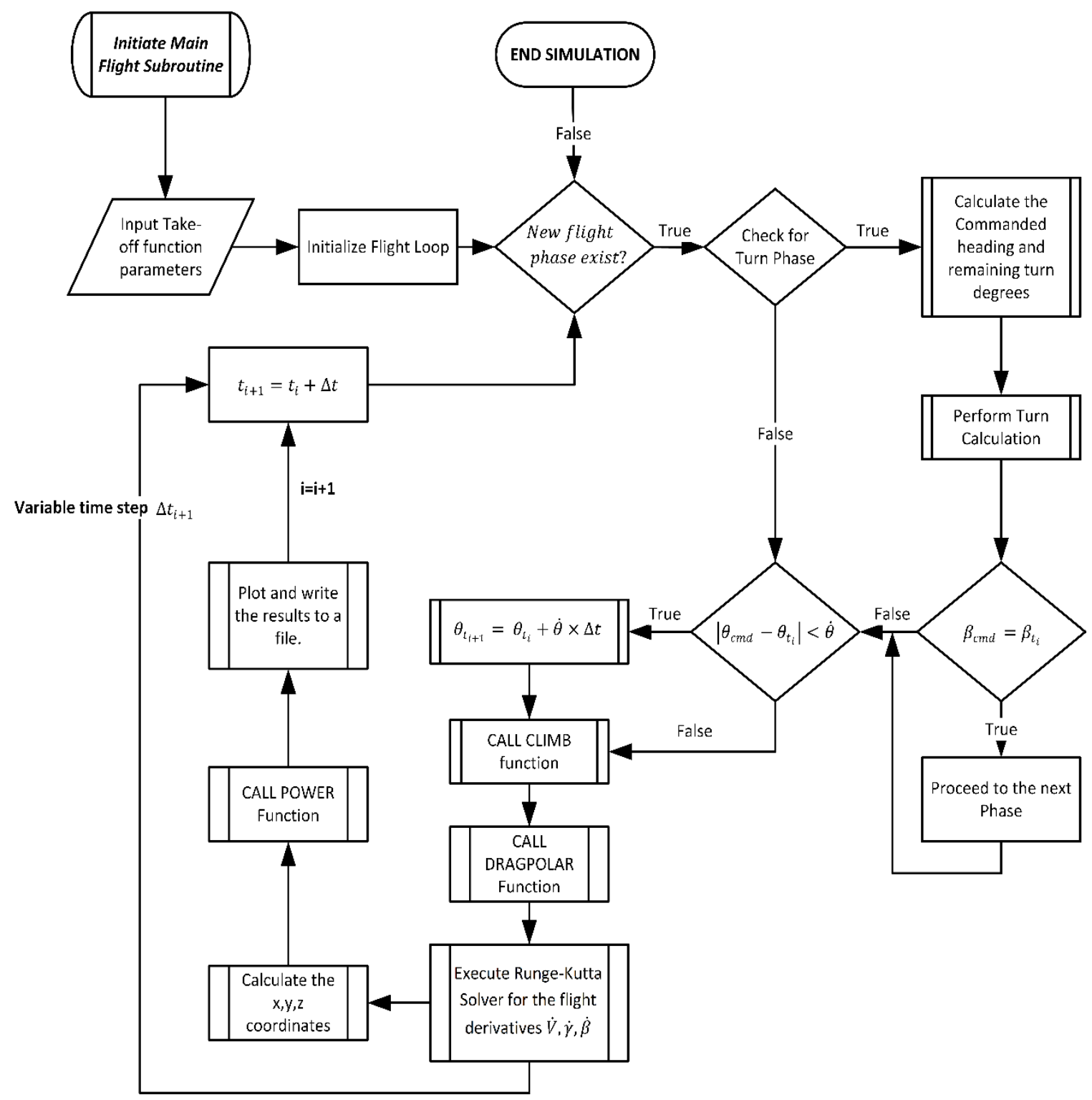

Figure 5.6 Flight subroutine flowchart

After the take-off subroutine is completed, the solution vectors are transferred to the flight module of the code. The flight subroutine then takes these parameters as input files for the next 
part of the simulation. This portion includes the rest of the flight phases: climb, cruise and turn segments.

Flight subroutines take over the simulation when lift-off has occurred and immediately checks whether the next flight phase exists and initiates the corresponding functions in the while loop.

The turn check occurs at the very beginning of the code; if there is a turn commanded, the code reads the angle of the turn and logs the remaining heading difference between commanded heading and the current heading until it reaches the certain small value (tolerance), and the turn control algorithm is applied by manipulating the bank angle of the aircraft.

After the turn check is completed or the turn is not executed, the longitudinal control part of the code checks the pitch angle. If the pitch angle is below the commanded value, it increases the pitch by time step times the pitch change rate shown at the flowchart above this then calls a climb or descent function. Briefly, this function checks for proximity to the cruise level. As described in Section 3.3.1 the pitch control algorithm kicks in and slowly adjusts the pitch rate to level off at a certain altitude.

Next, drag and lift coefficients of the aircraft at the current state are obtained with the dragpolar function. Then the solver is run and the next time step solution is generated. From this solution, power calculations are made for thrust, power and battery parameters for the next time step. Finally, results are plotted while the previous time step plots are stored and the solution text file is updated. Finally, the simulation proceeds to the next time step suggested by the solver. 


\subsection{Simulation Segments and Functions}

The following subsections explain the functions used in the simulation code with their respective inputs and outputs into the simulation.

\subsubsection{Main Subroutines}

Two functions contain the main simulation and are given by the arrow bullets. Detailed definitions of take-off and flight missions are shown.

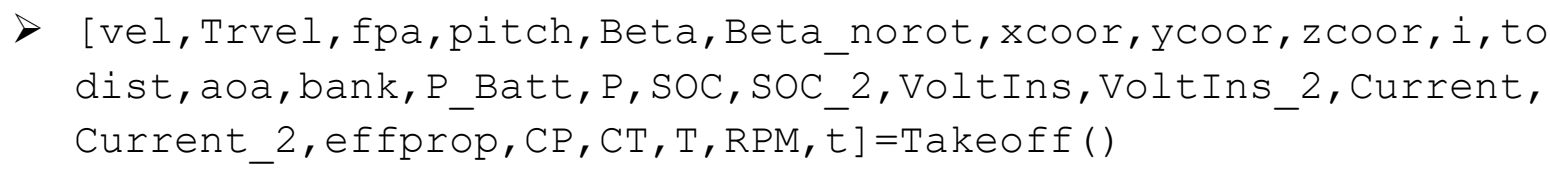

Inputs: Globally defined

Outputs: Outputs shown respectively;

[Velocity, True velocity, Flight path angle, Heading, xyz, coordinates, time indice, Take-off distance calculated, Angle of attack, Bank angle, Battery Power, Required Power, SOC(Constant battery method), SOC(SIMULINK estimation), Battery voltage, Battery Voltage(SIMULINK), Current draw, Current draw(SIMULINK), Propeller efficiency, Propeller power coefficient, Propeller thrust coefficient, Thrust, Propeller RPM, Time]

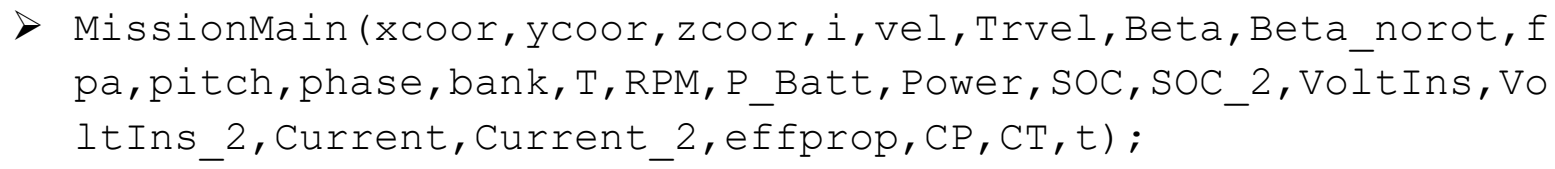

Inputs: Outputs from Take-off subroutine.

Outputs: Plotted and written to file; 


\subsubsection{Subsystems}

Subsystems are defined in the following bullet points:

I. Drag Polar calculation module: $[\mathrm{CD}, \mathrm{CL}]=$ Dragpolar (aoa, Uinf)

This calculates the drag and lift coefficient for the given angle of attack and the speed of the aircraft using the lookup tables.

Inputs: Angle of attack and Velocity

Outputs: Lift and Drag coefficients

II. Propulsion and Energy systems calculation module:

[P, P_Batt, T, SOC,SOC_2, Vterminal,Vterminal_2, effprop, CP, CT , Current, Current_2, $\overline{R P M}]=$ Power (Vel, CL, D_unit, t, SOC, SOC_2, RPM)

This calculates the power generated by the battery and required by the motor/propulsor combination. Also, state of charge and instant voltage levels are computed with constant power and the SIMULINK Simscape ${ }^{\mathrm{TM}}$ battery model. Propeller coefficients are calculated in this module.

Inputs: Velocity, Lift coefficient, Drag coefficient, time, Pervious SOC values and RPM

Outputs: Power values for propeller/motor and battery, Thrust, Terminal voltage of the battery, Propeller coefficients, Current drawn from the battery, RPM

III. Climb and descent module:

[pitch ]=Climbdescent (aoa, vel,fpa, h, zcoor, fparate, T, bank, D _unit, pitch) 
The climb and descent module manipulate the pitch angle of the aircraft when the current altitude is close to the cruise altitude set. This is performed by the pitch control equation used in Section 3.3.1.

Inputs: Angle of attack, Velocity, Flight path angle, time step, z coordinate (altitude), flight path angle change rate, Thrust, bank angle, Drag coefficient, Pitch angle

Outputs: Pitch angle

\section{Turn module:}

[bank, rturn] =Turn (vel, bank, T, RemDeg, Cmd_HDG, turnrate, Beta, Dir)

Turn is being performed in this module using the relation obtained in the heading control in Section

3.3.2.

Inputs: Velocity,bank angle, Remaining turn degrees, Commanded Heading angle, Turn rate, Heading, Direction of the run

Outputs: Bank angle, Turn rate

\section{Solver module:}

[f_val] =Rungekutta (time, vel, gamma, head, h, aoa, T, CL, D_unit, bank)

Solver calculates the derivatives of each EOM for the next time step.

Inputs: Time, Velocity, Flight Path angle, Heading, Angle of attack, Thrust, Lift and Drag coefficient, Bank angle

Outputs: Derivates of the EOM

\subsubsection{Other Functions}

I. Read the Aerodynamics charts and store it globally: Aero3D ( ) ; 
Inputs: Globally defined

Outputs: Globally defined

II. Read the input file "Inputfile_PFTool": readXLS (xlsname) ;

Inputs: Name of the excel input file

Outputs: Globally defined

III. Select propeller from the input: Propselect ( ) ;

Select the propeller and read the $C P, C T, \eta$ from the file.

Inputs: Globally defined

Outputs: Globally defined

IV. Fit polynomial function to the propeller point data and plot: PropMotor ()

Inputs: Globally defined

Outputs: Globally defined

V. Construct Constant Battery Discharge method: traub_clps_battery()

Inputs: Globally defined

Outputs: Globally defined: n (curve fit), voltage_fit (gives the voltage with current)

VI. Simulink Battery model for the validation:

[SOC,Vterminal]=Simulink_Battery_Model (tstart, tend, SOCold,Amp)

Inputs: Starting time, End Time, previous SOC value, Battery current draw

Outputs: SOC, Terminal Voltage 


\section{CHAPTER 6}

\section{RESULTS \& DISCUSSION}

This chapter will present the results of the simulations that were performed in different configurations. Various scenarios will be tested in the analysis of the trajectories generated by the Mathematical 5-DOF system. Observing these different flight mission scenarios provides insight into the principles of these simulations. The conditions will be investigated in the first section of the analysis portion; then the entire simulation will be employed by exploring all the capabilities

of the simulation including climb and turn functions. The following section will present the contrasting factors and the effects of these factors on the parameters such as simulation time, battery drain rate and the aircraft performance in climb and turn segments.

Each of the simulations focuses on the comparison of the RPM value of the propeller used in the simulation. The main objective is to observe the difference between the different propeller settings throughout the flight regime and keep the propeller speed constant to give the solution with different thrust coefficients effectively changing the performance parameters of the UAV for each flight.

\subsection{Cruise Height Benchmark Analysis}

In this section, results from the simulation conducted in the following conditions are presented. First, the trajectories initiated with the state of charge of "1". This assumes a fully charged battery model used for the calculation of the battery performance. We consider the minimum allowable remaining SOC to be 0.5 . This gives the margin of $50 \%$ battery capacity, 
enough for the aircraft to perform a loiter and land before experiencing critical damage to the internal components of the battery by discharging excessively before the mission finishes.

In this trajectory simulation the range and endurance of the mission were analyzed. The common motion on both comparison analyses are defined as follows:

1. Aircraft takes-off from runway with friction coefficient of $\xi=0.04$. This is a standard value for asphalt runway conditions.

2. Next, Aircraft accelerates to the take-off speed dictated by the positive flight path angle.

3. Pilot commands a $8^{\circ}$ pitch angle with a constant rate of $8^{\circ}$ per second.

4. Climb profile established and checked for level climb.

5. The pitch control algorithm takes over the control of the pitch angle and slowly levels off the aircraft to the cruise altitude selected.

6. Flight is continued until the SOC hits 0.5 value then the results are generated.

These steps are followed for four different RPM values: 8000, 9000, 10000, 11000. These RPM values provide the most realistic control of the propulsion system in the AVISTAR RC plane. Finally, these conditions are followed for the cruising altitudes of $100 \mathrm{~m}$ and $200 \mathrm{~m}$ and comparison is made for the flight parameters obtained from the simulation. Since there is no lateral motion present in this test, the flight path can be analyzed in a two-dimensional plot and is shown in Figure 6.1. 


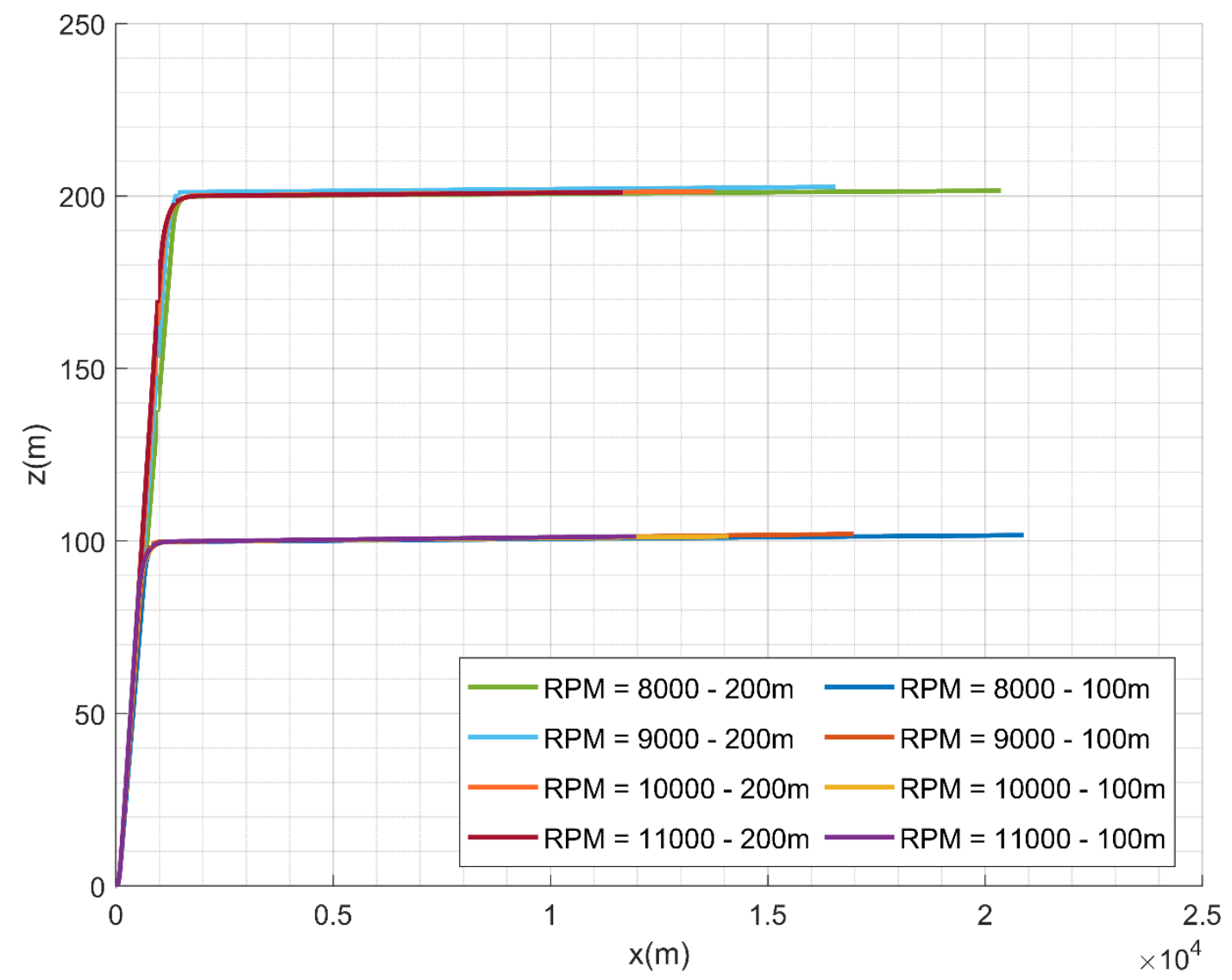

Figure 6.1 Trajectory of the Straight flight mission for $100 \mathrm{~m}$ and $200 \mathrm{~m}$ cruising altitudes

The maximum range solutions occurred at 8000RPM, providing 20,898 meters of range for 100m cruise altitude. The $200 \mathrm{~m}$ cruise altitude mission yielded a slightly lower value of 20,366 meters. A similar trend was seen for other RPM values, but the difference was greater for lower RPM values. 


\begin{tabular}{|c|c|c|c|c|}
\hline Cruise/RPM & 8000 & 9000 & 10000 & 11000 \\
\hline $100 m$ & $20898 m$ & $16981 m$ & $14113 m$ & $11969 m$ \\
\hline $200 m$ & $20366 m$ & $16550 m$ & $13758 m$ & $11667 m$ \\
\hline Difference $=$ & $532 m$ & $431 m$ & $355 m$ & $302 m$ \\
\hline
\end{tabular}

Table 6.1 Range values for each set of simulation

The climb phase of the simulation was computed by adjusting the pitch angle of the aircraft and consequently altering the angle of attack. Flight path angle, which is the angle the aircraft climbs through the air is also a byproduct of the relation of these flight angles. From Figure 6.2 to Figure 6.5, flight attitude angles are given from the series of simulations correlating to the motor RPM values.

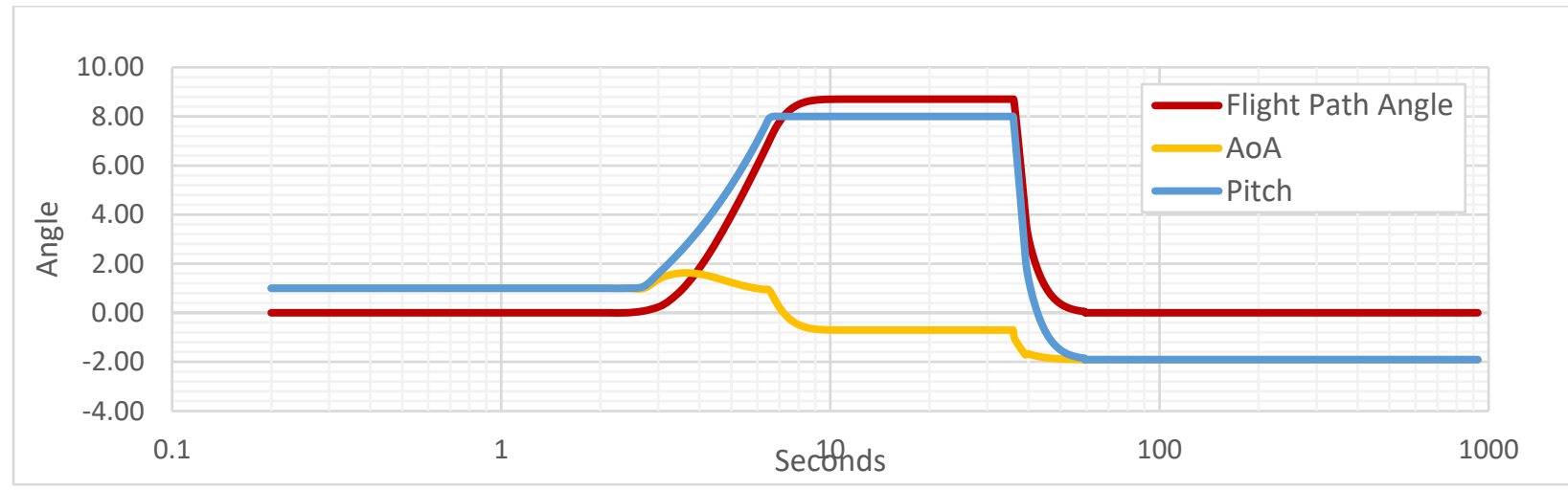

Figure 6.2 Flight attitude angles for simulation RPM =8000 for $100 \mathrm{~m}$ cruising altitude 


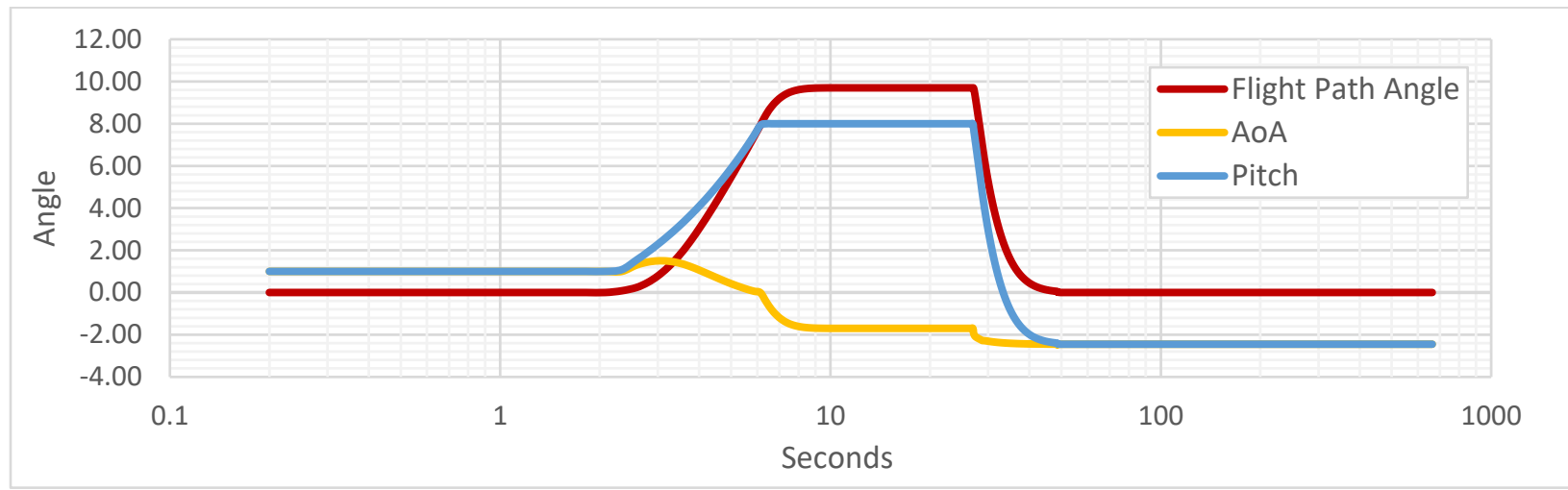

Figure 6.3 Flight attitude angles for simulation $R P M=9000$ for $100 \mathrm{~m}$ cruising altitude

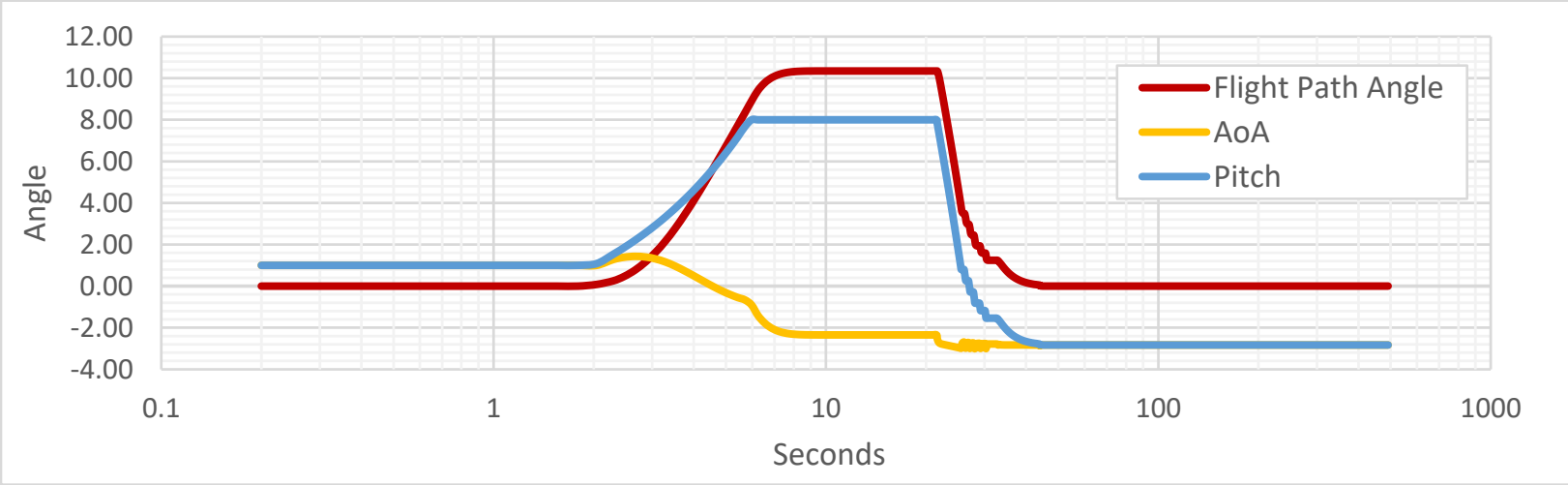

Figure 6.4 Flight attitude angles for simulation $R P M=10000$ for $100 m$ cruising altitude 


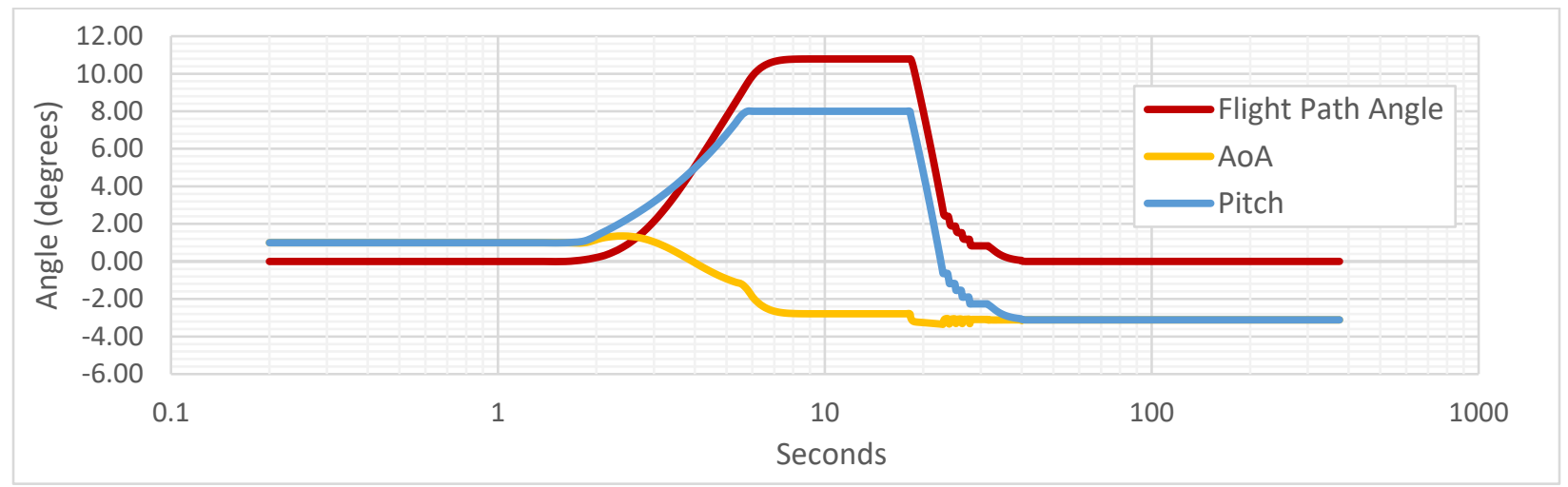

Figure 6.5 Flight attitude angles for simulation $R P M=11000$ for $100 m$ cruising altitude

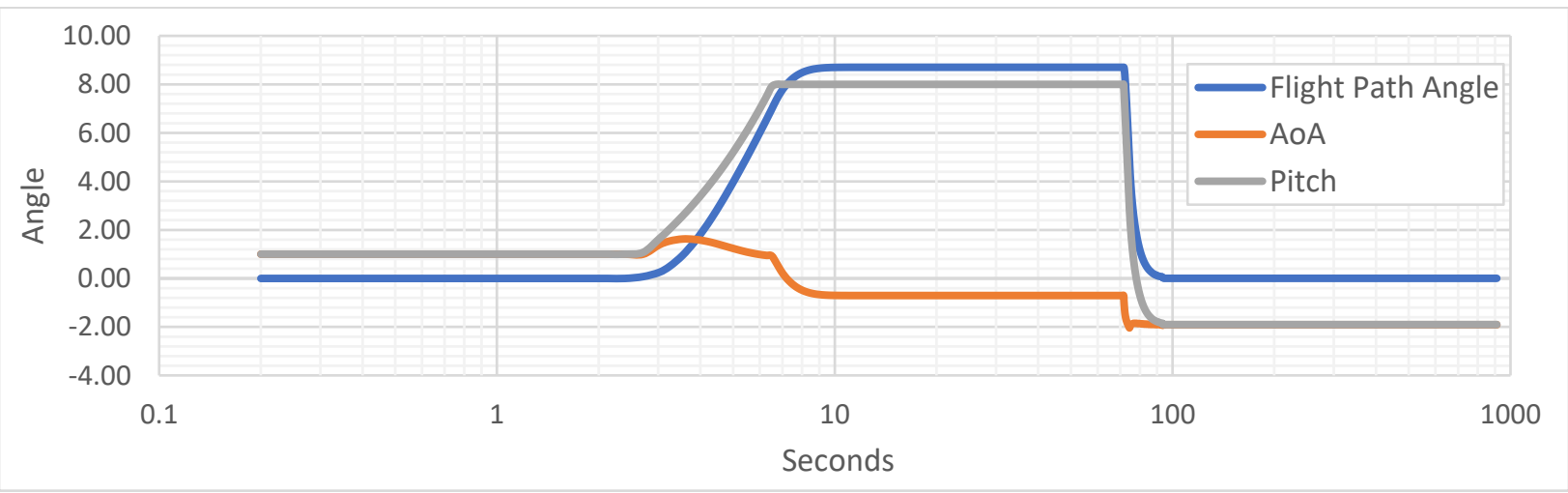

Figure 6.6 Flight attitude angles for simulation $R P M=8000$ for $200 \mathrm{~m}$ cruising altitude 


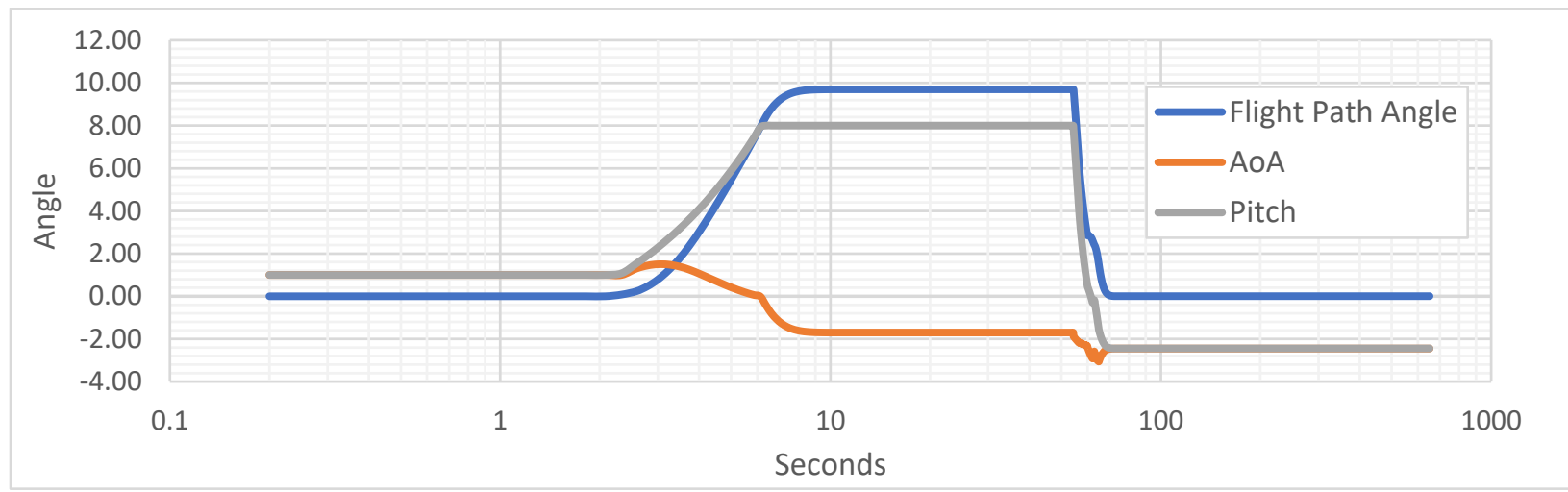

Figure 6.7 Flight attitude angles for simulation $R P M=9000$ for $200 \mathrm{~m}$ cruising altitude

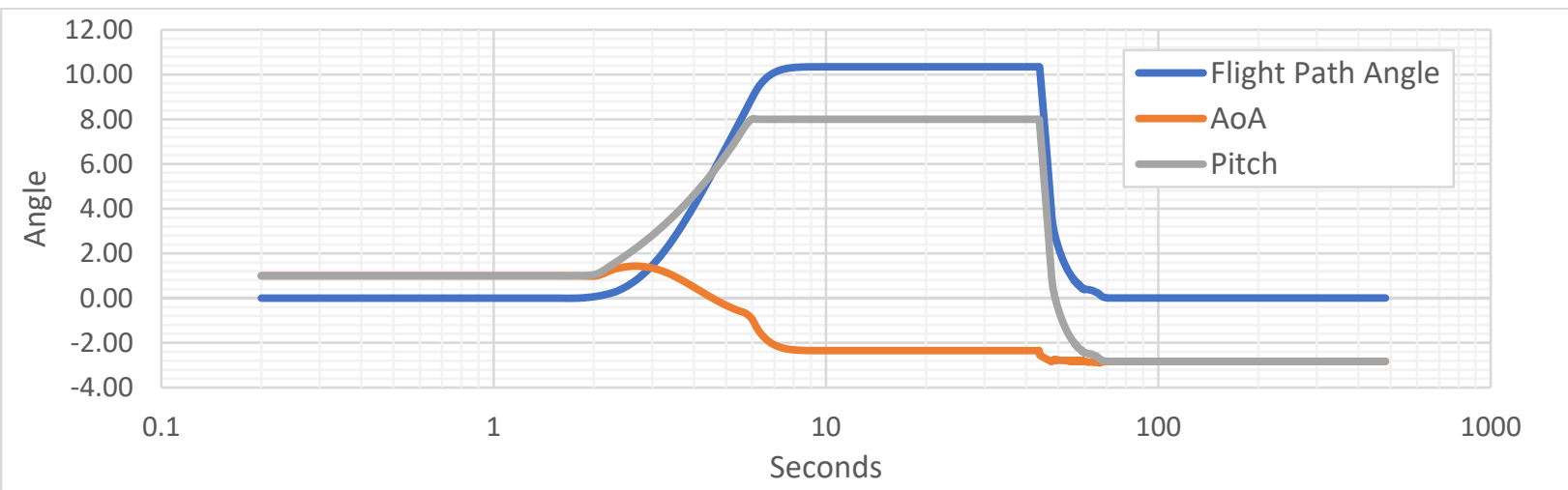

Figure 6.8 Flight attitude angles for simulation $R P M=10000$ for $200 m$ cruising altitude 


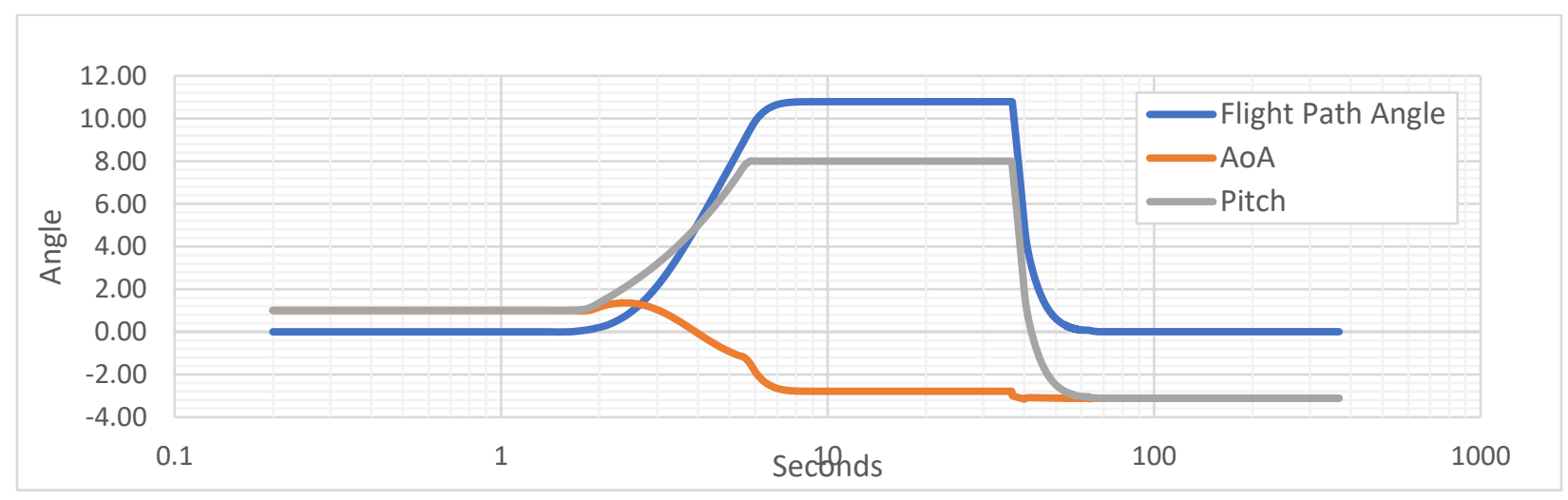

Figure 6.9 Flight attitude angles for simulation $R P M=11000$ for $200 \mathrm{~m}$ cruising altitude

For each RPM value, the simulations are compared and contrasted with two different cruise levels, and results show little difference from each other for different RPM values with a more significant shift seen in the different cruise levels. Next, a portion of the results study is to investigate battery parameters. SOC change throughout each simulation is calculated through the methods described in the previous chapters. 


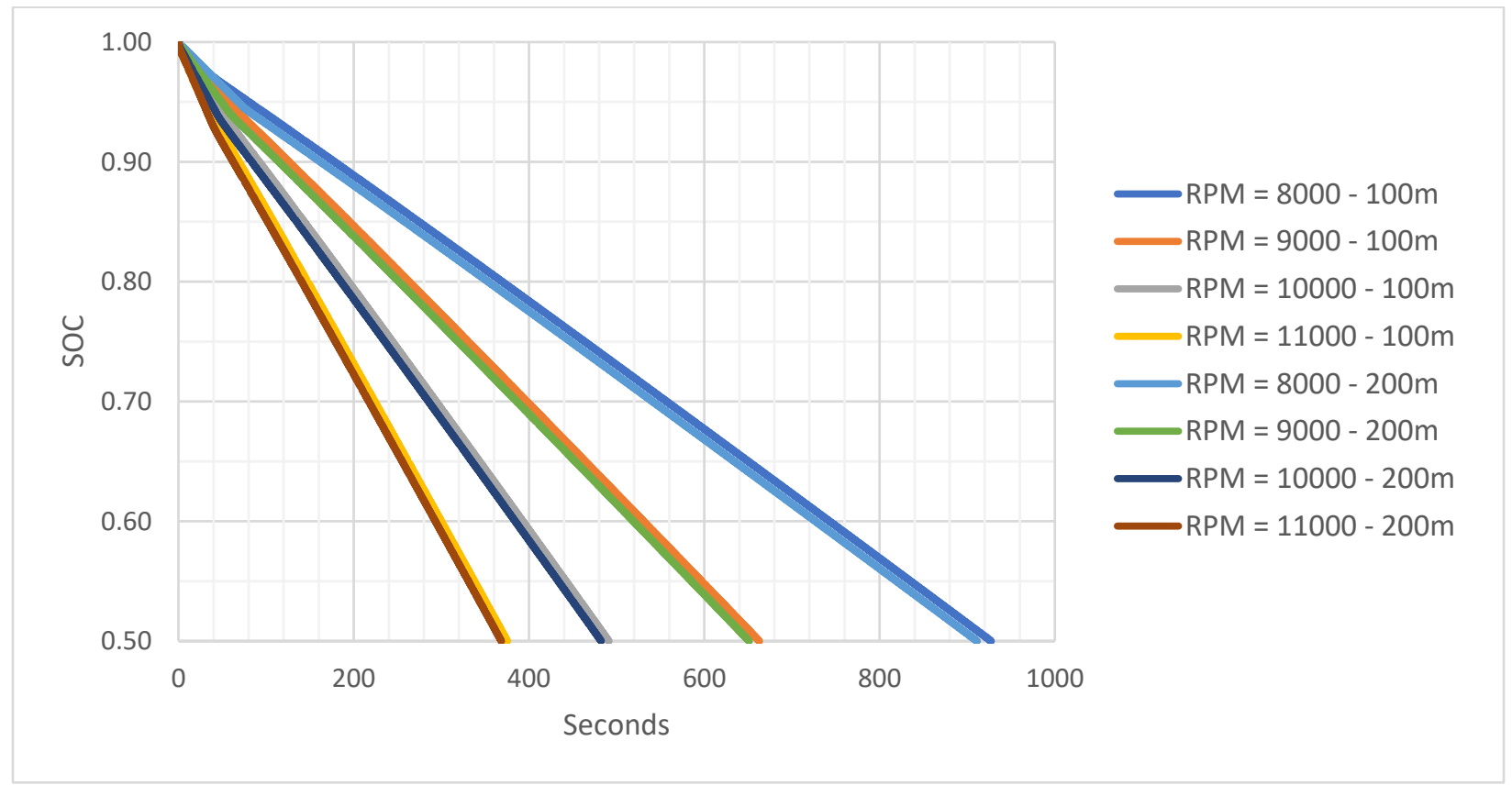

Figure 6.10 SOC vs Time for four RPM settings in 100m or 200m cruising altitudes

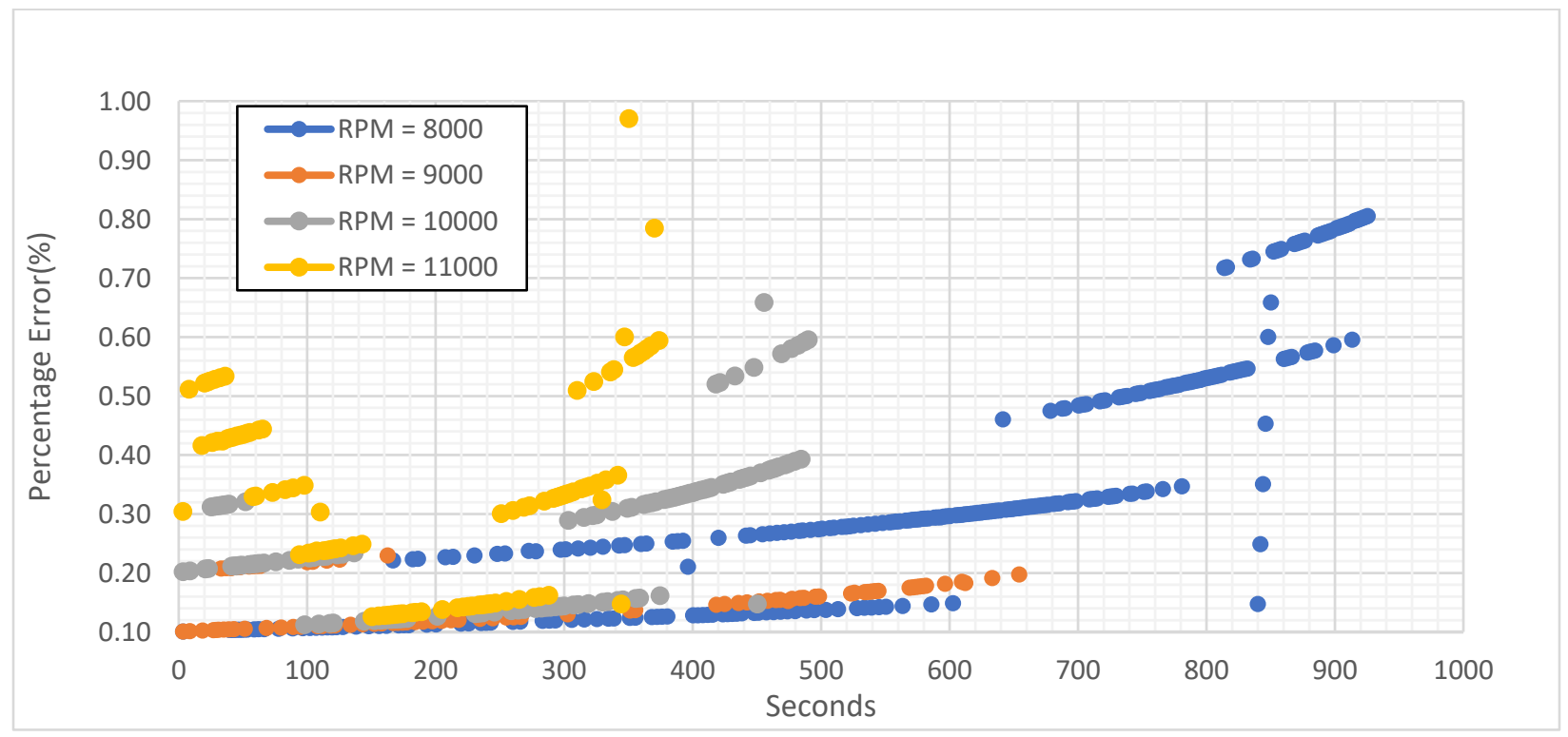

Figure 6.11 SOC Error, Traub's constant discharge method vs. SIMULINK model 
SOC values are calculated with the constant power discharge method which is an intelligent way of calculating the SOC and voltage values of the battery while the power taken from the system is mostly constant, but the current draw is not as the voltage drops as SOC drops. As can be seen, there is an upward trend in current drawn as the time increases. Also, more current will be drawn at the cruising segment of the flight for higher RPM. The following plots show the current drawn from the battery with respect to the time of the simulation. The initial large changes are due to the takeoff phase.

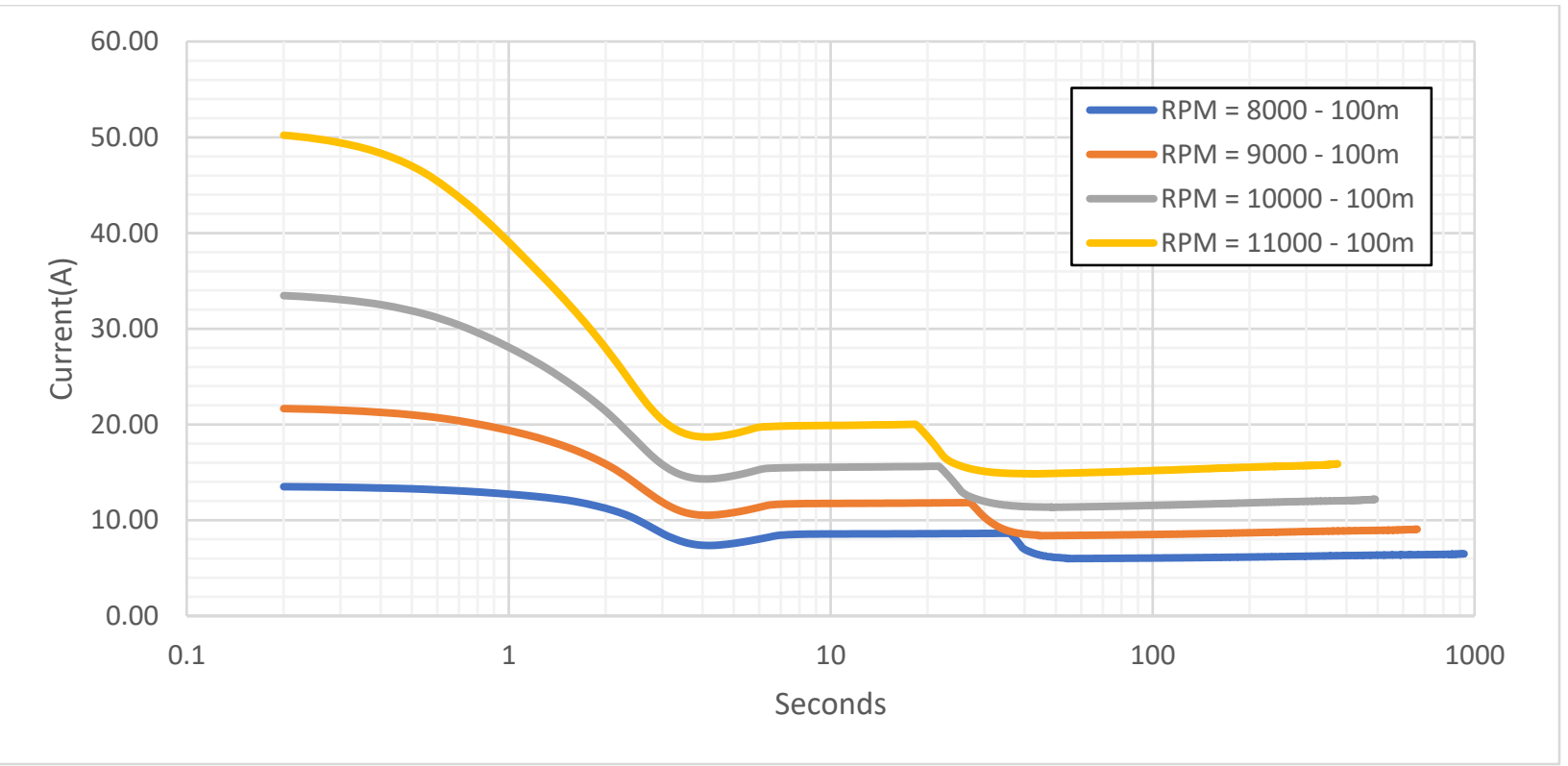

Figure 6.12 Current drawn vs time plot for 100m cruising altitude 


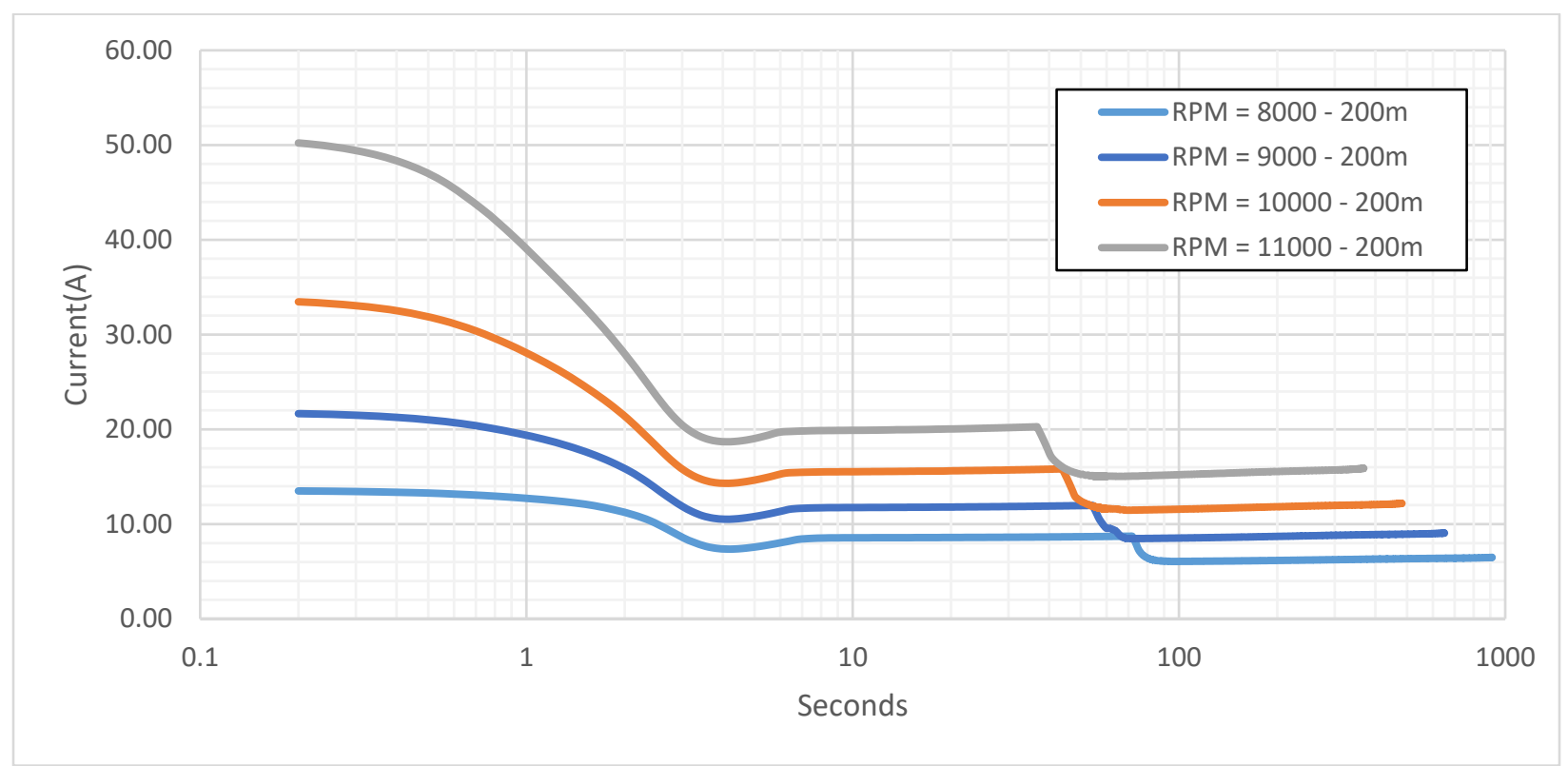

Figure 6.13 Current drawn vs time plot for 200m cruising altitude

Afterwards, the take-off current draw drops. Finally, current levels off when the aircraft is flying straight and level at cruising altitude, and as the battery is depleted the current rises gradually as voltage sags to hold constant power.

The voltage is influenced by the thrust that the propeller generates and in turn the current from the battery. Intuitively, higher thrust values yield higher voltage drop rates for the battery. Due to the limitations of this method, the final voltage cannot be obtained as measured experimentally due to the voltage after discharge being cut off. 


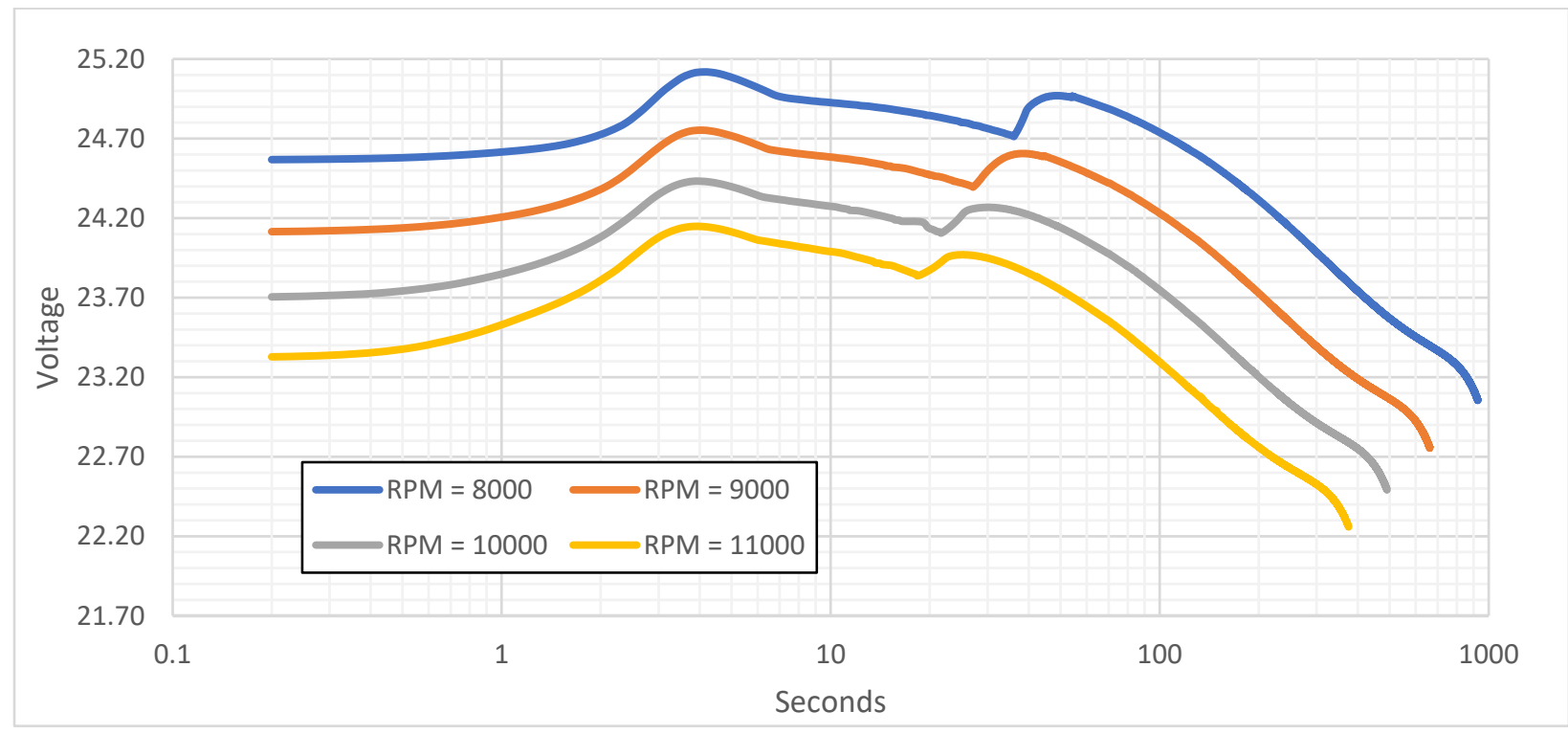

Figure 6.14 Voltage vs Time for four RPM settings at 100m altitude

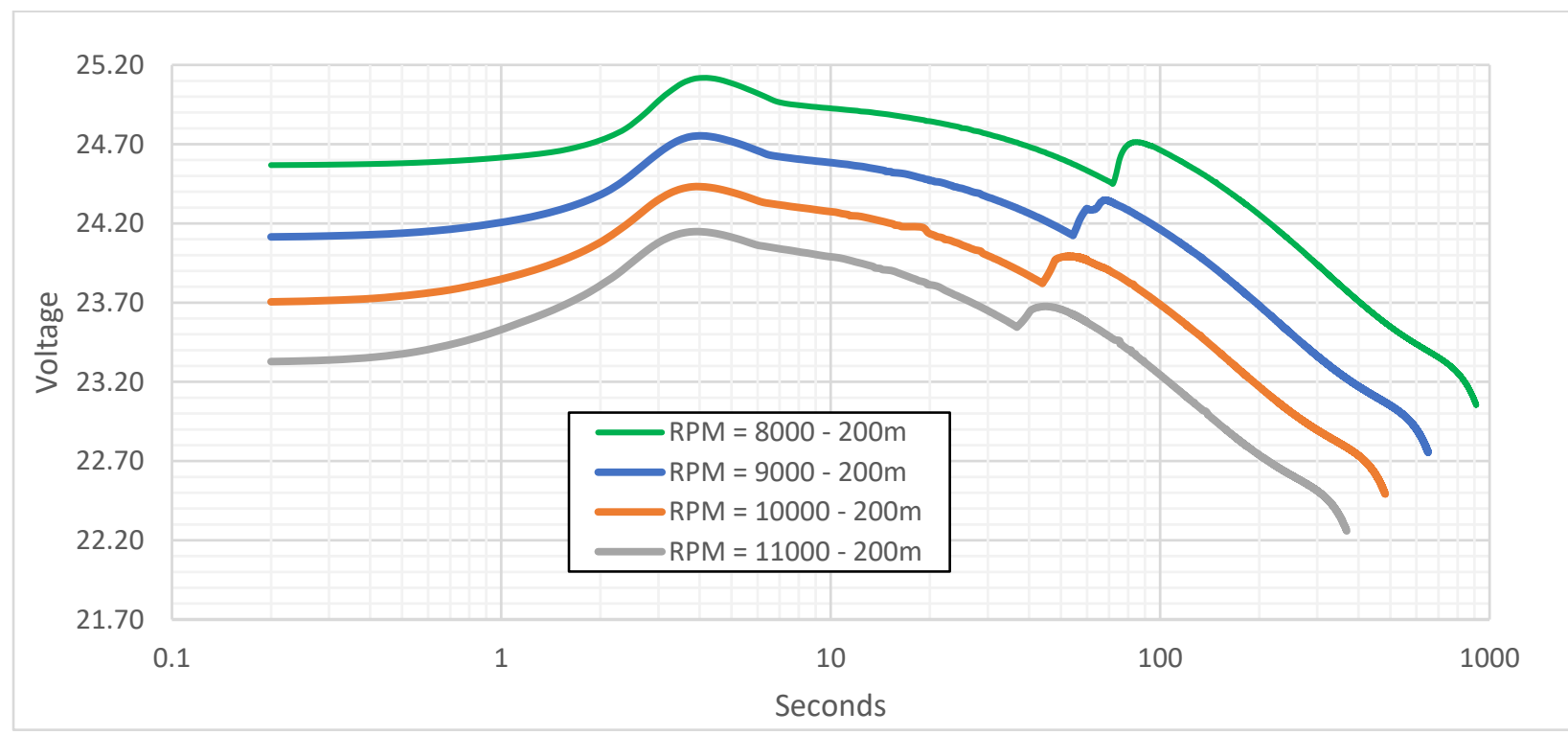

Figure 6.15 Voltage vs Time for four RPM settings at 200m altitude 
Velocity profiles show the take-off run calculation which is seen as a steep curve at the first few seconds of the simulation. The steep increase is interrupted by the pitch angle commanded by the control methods. The final value is reached when the aircraft levels off at the cruising altitude.

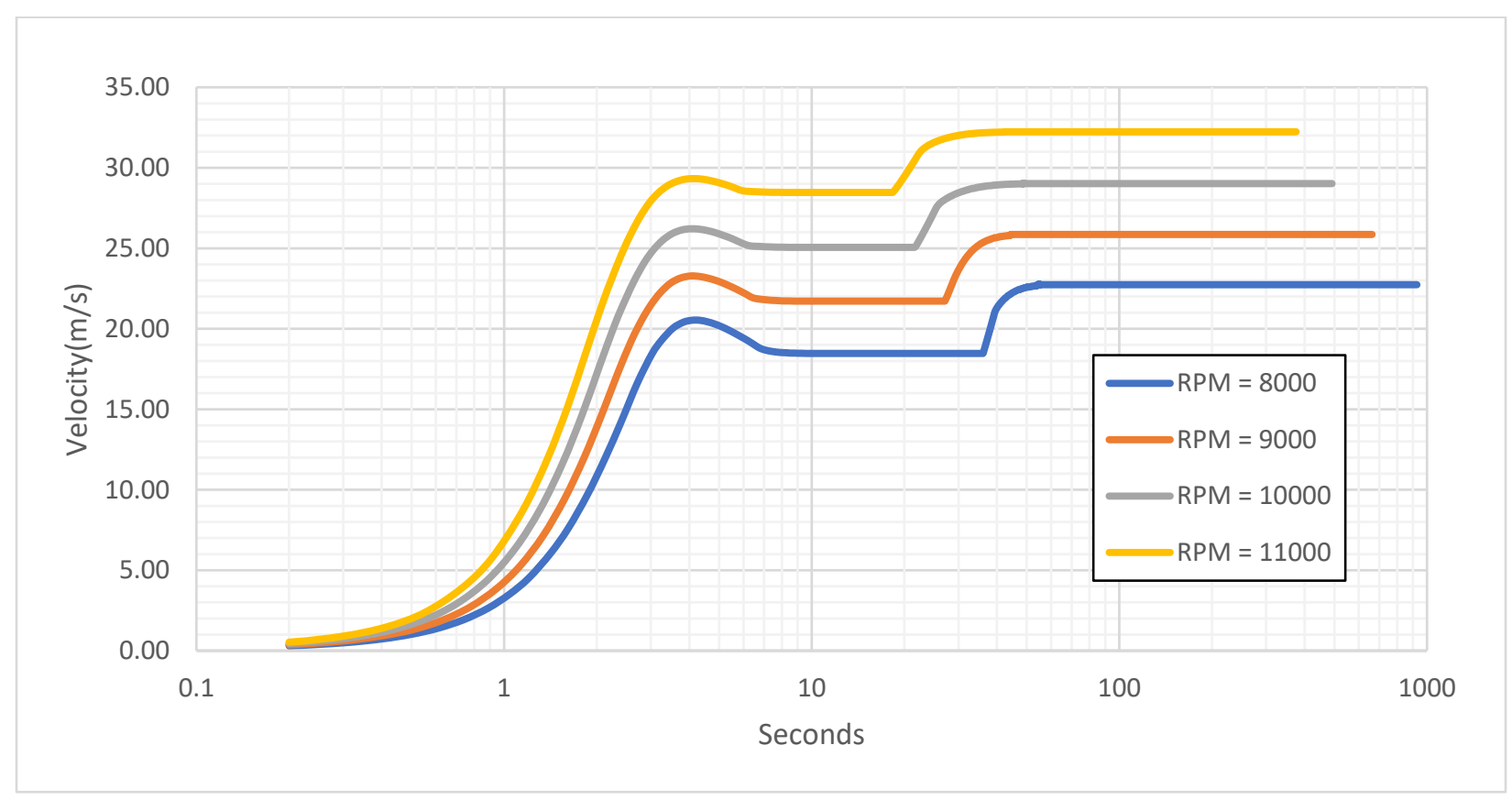

Figure 6.16 Velocity vs Time for four RPM settings in 100m or 200m cruising altitudes

Thrust values are calculated using the propeller data. The main condition affecting the thrust values for a given RPM is the velocity. Power is also one of the parameters significant to the simulation. We consider two separate representations for the power. One of which is the power that the propeller produces that comes with the use of propeller coefficients described in Section 
3.4.1. The other power is called the "battery available power," and it is the relation from the combination of the total efficiencies of the drivetrain multiplied with the maximum current defined by the limitations of the battery. In our case, the maximum operational limit for the battery used in this simulation is $60 \mathrm{~A}$. This is set by looking at the manufacturer notes and opting for the optimal safe value for the max sustainable current draw for the battery.

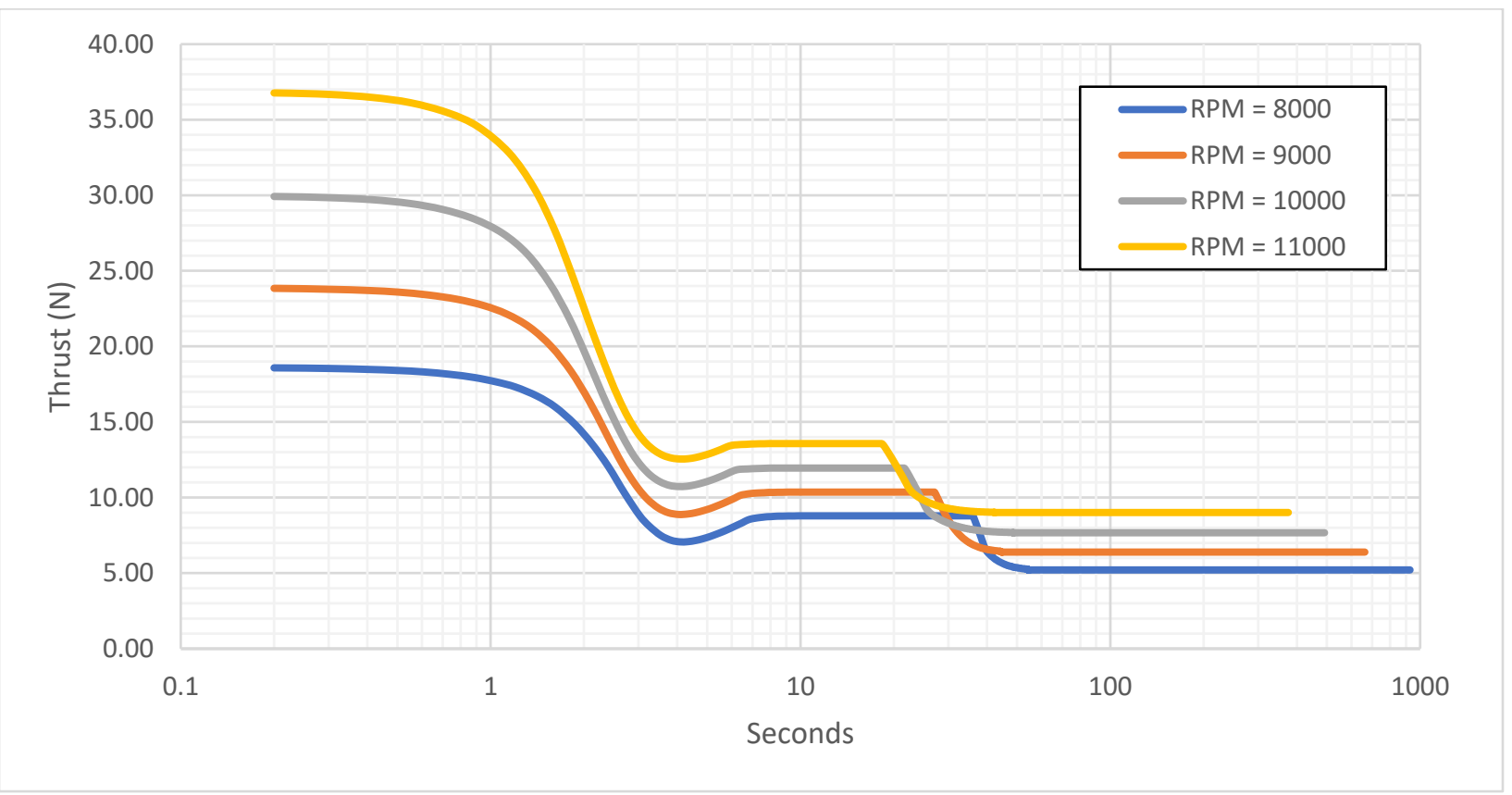

Figure 6.17 Thrust vs Time for four RPM settings in 100m or 200m cruising altitudes 


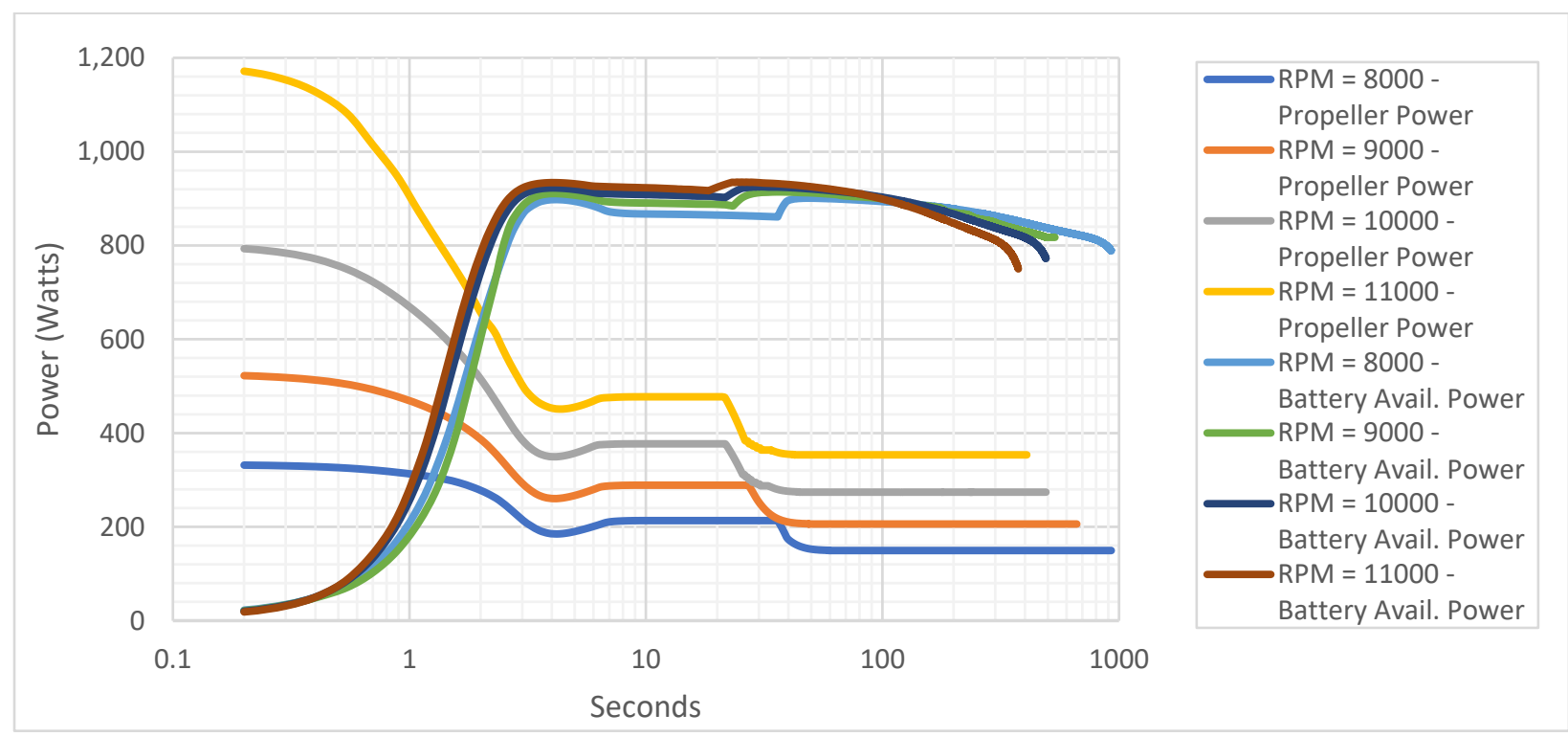

Figure 6.18 Power vs Time for four RPM settings in 100m

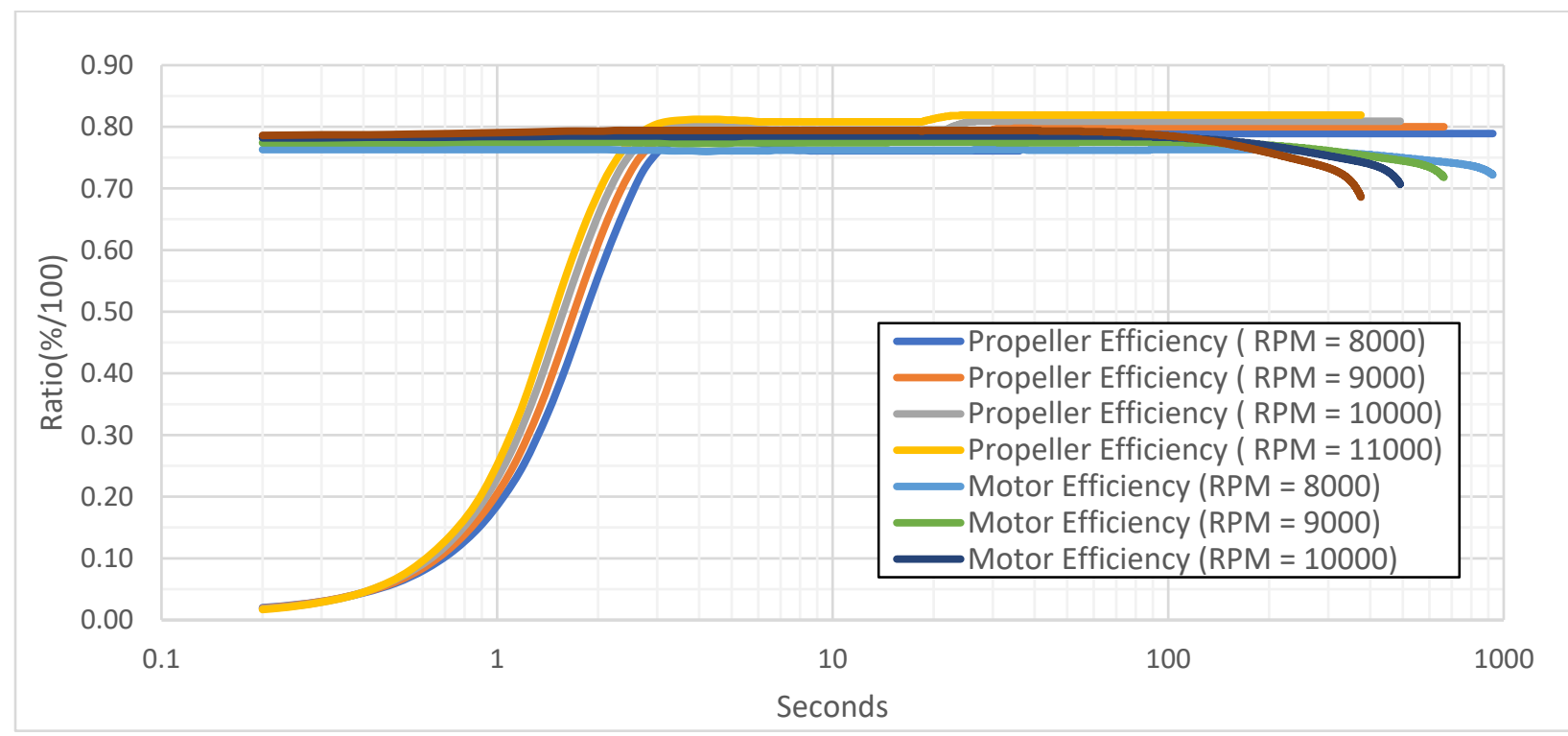

Figure 6.19 Efficiencies of the drivetrain components with time at 100m cruising altitude 
Finally, the computing performance for each simulation is shown in Figure 6.20. It is clear that flight simulations with the longest simulation time had the slowest run time. Also, the $200 \mathrm{~m}$ cruise level is faster than the $100 \mathrm{~m}$ cruise level simulations in all cases except the "11000" RPM value.

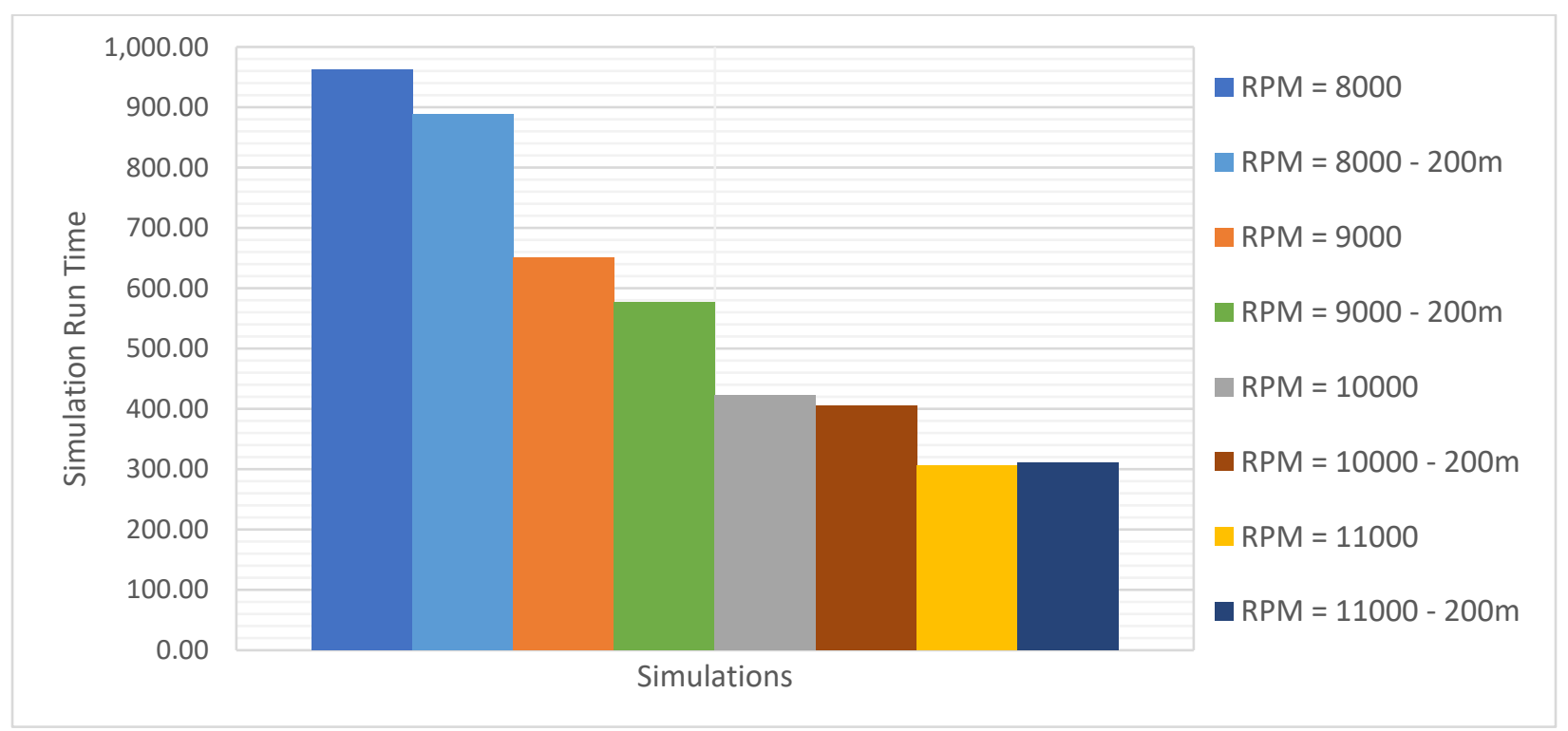

Figure 6.20 Computation time for all simulations

\subsection{Turning Mission Benchmark Analysis}

For the turning flight mission, the simulation utilizes the full 5-DOF system designed in this thesis. The sequence of commands and control are initiated with the order below for this mission: 
1. Aircraft takes-off from runway with friction coefficient of $\mu=0.04$. This is a standard value for asphalt runway conditions.

2. Next, Aircraft accelerates to the take-off speed dictated by the positive flight path angle.

3. Pilot applies the $8^{\circ}$ pitch angle with the constant rate of $8^{\circ}$ per second.

4. The climb profile is established and checked for constant climb.

5. The pitch control algorithm takes over the control of the pitch angle and slowly levels off the aircraft to the cruise altitude selected.

6. After the level-off is completed, the pilot banks the aircraft to the current bank angle (Left turn in this simulation) and continues the same final bank angle until the end of the mission.

7. Flight is continued until the SOC reaches the 0.5 cutoff value and then the results are generated.

Similar to the previous analysis, these steps are followed for four different RPM values: $8000,9000,10000,11000$. However, this time the trajectory of the aircraft is generated in threedimensional space. 


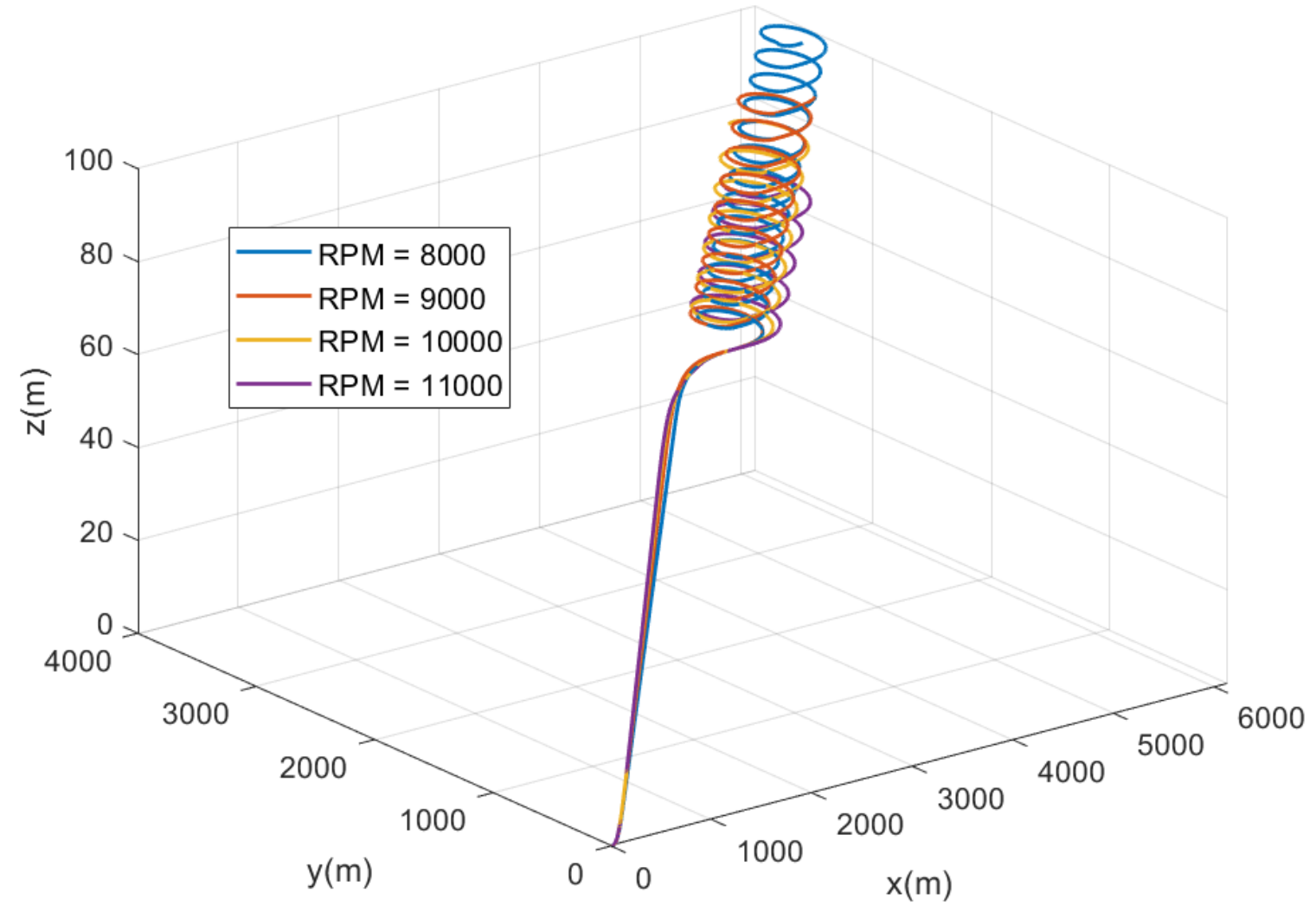

Figure 6.21 Turning Mission Trajectory

As seen from Figure 6.21 each flight is completed with the continuous segment of turn while keeping the same altitude of $100 \mathrm{~m}$. In the following figures the velocity profile for each RPM value is given, and it shows a similar trend to the previous mission. Bank angle for each simulation is also obtained from the turn control module implemented in the code; hence, different thrust levels are changing the bank angle thereby changing the turn performance of the aircraft. 
Figure 6.22 Velocity Profile of the Turning missions ranging RPM values

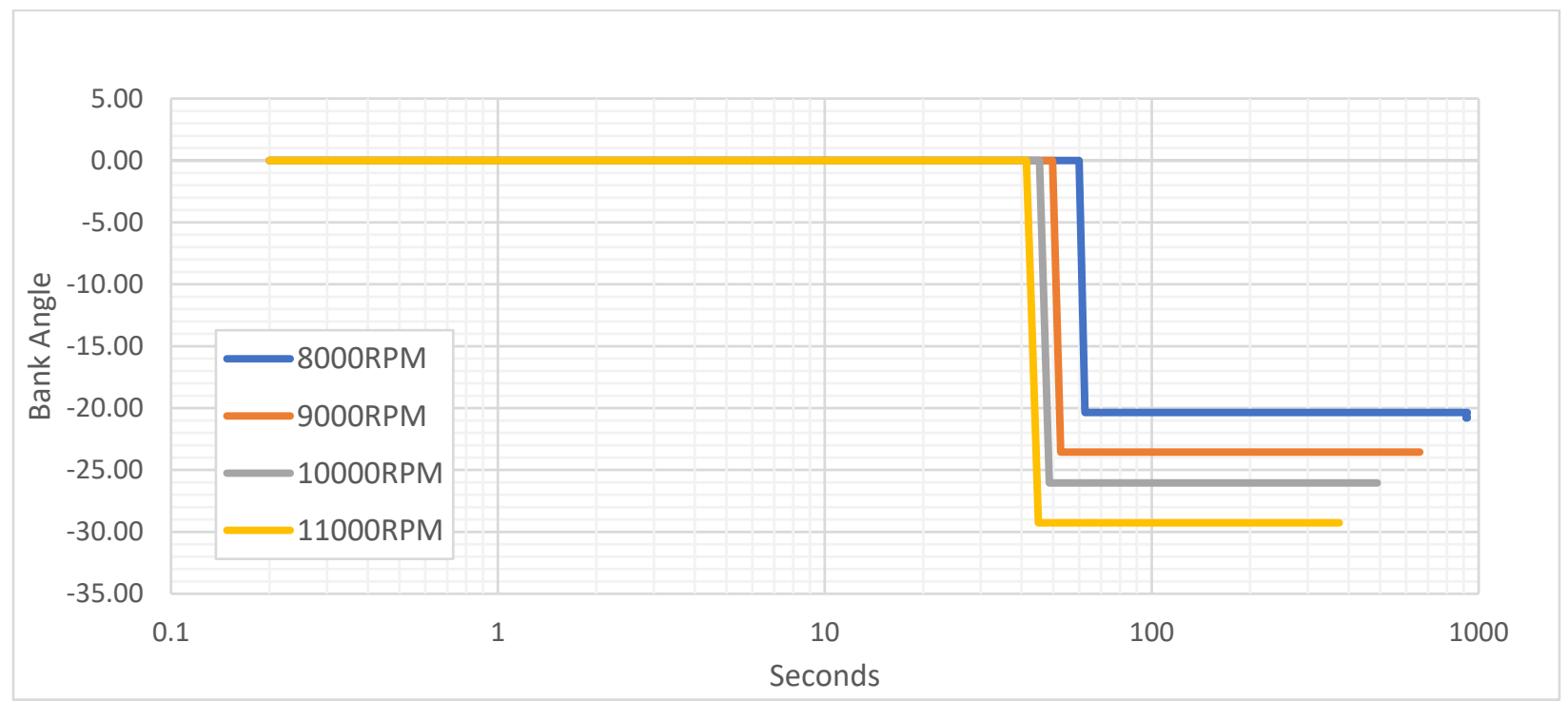

Figure 6.23 Bank angle vs Time for each RPM values 
Similar to the first mission, flight attitude angles are collected from the simulation for each RPM flight mission shown from Figure 6.24 to Figure 6.27.

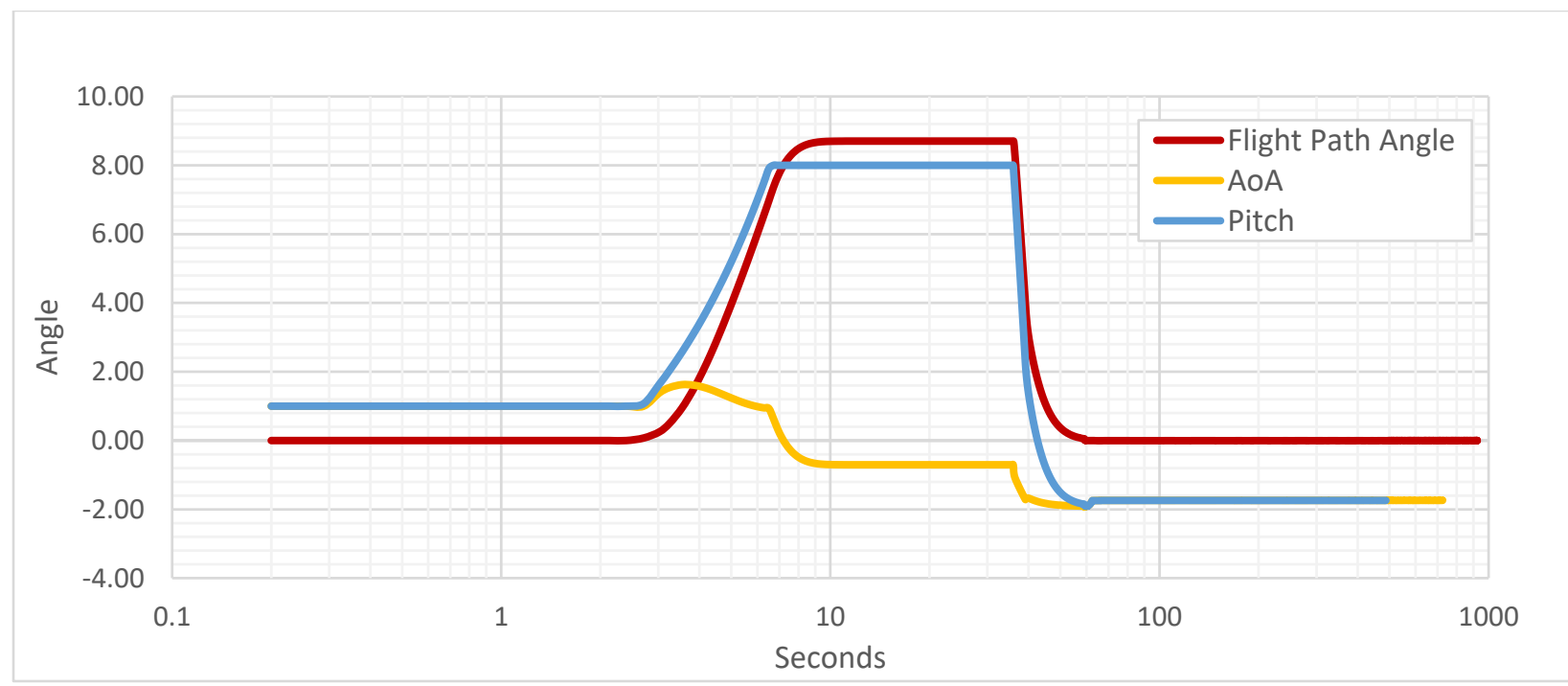

Figure 6.24 Flight attitude angles for simulation $R P M=8000$ 


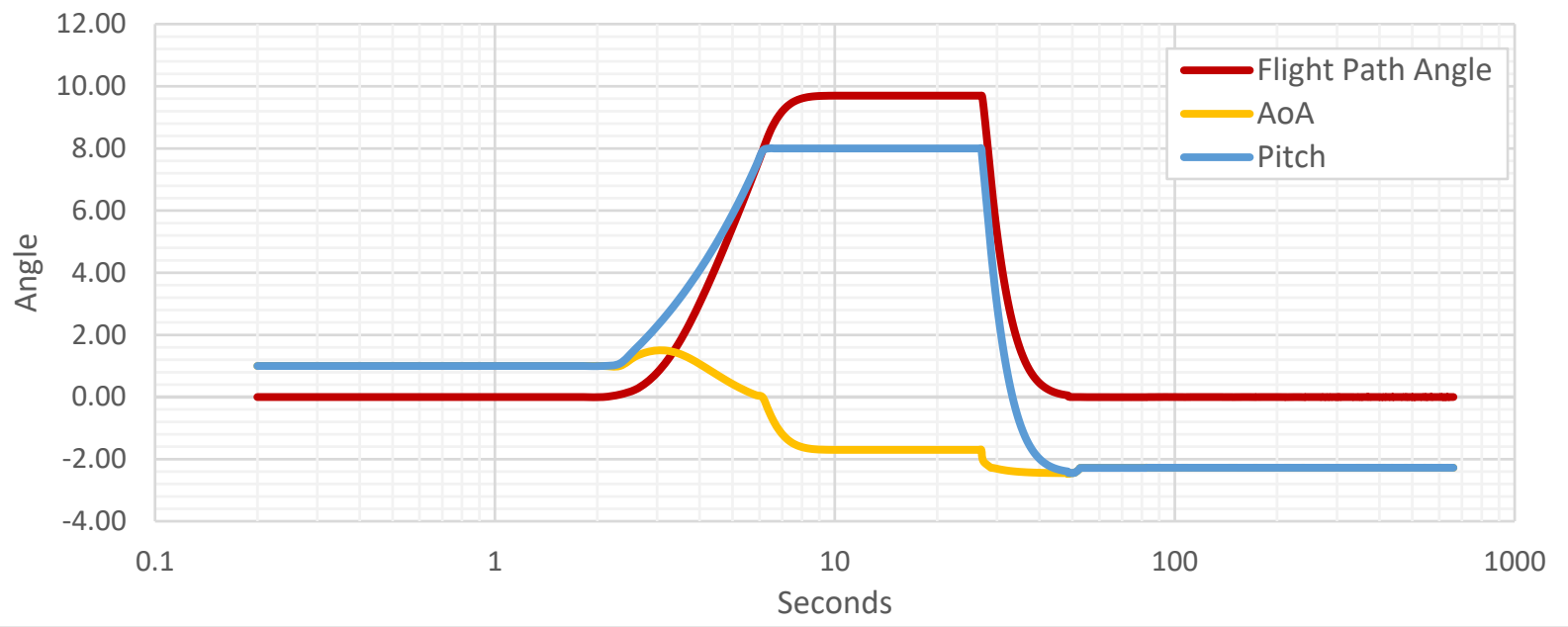

Figure 6.25 Flight attitude angles for simulation $R P M=9000$

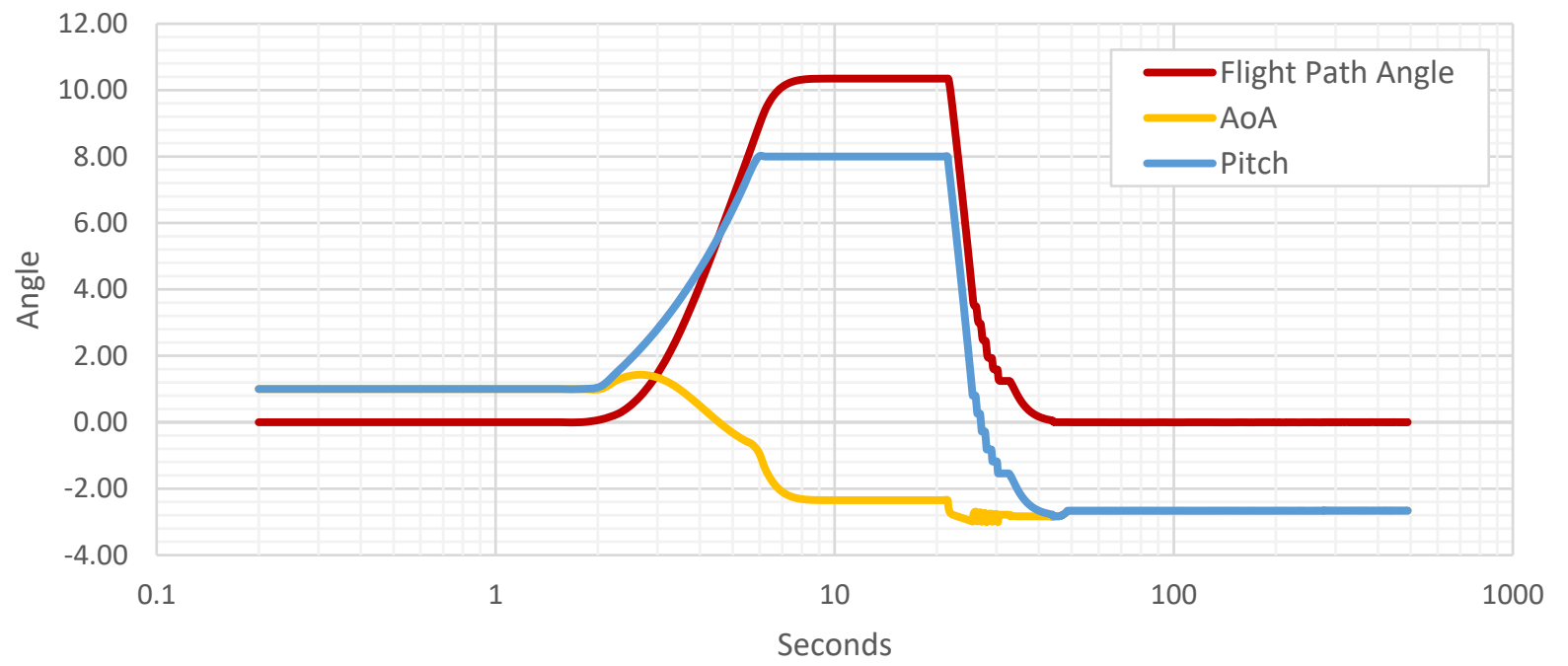

Figure 6.26 Flight attitude angles for simulation $R P M=10000$ 


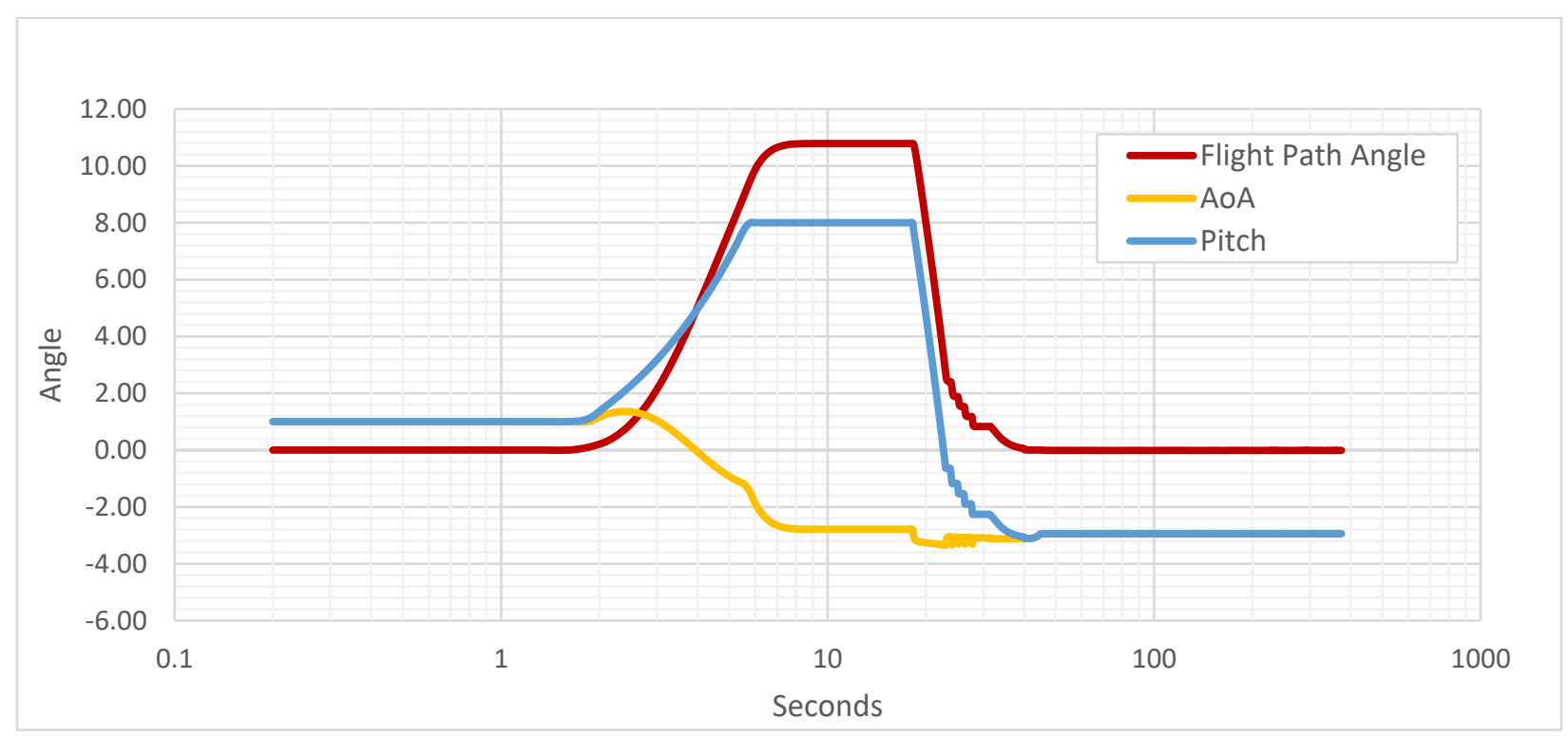

Figure 6.27 Flight attitude angles for simulation RPM $=11000$

Figure 6.28 to Figure 6.30 show the SOC, current and the voltage values obtained for the turning mission for the aircraft. The fastest battery drain is seen for the "RPM $=11000$ " simulation for approximately 344 seconds, and the longest endurance obtained for "RPM $=8000$ " was for approximately 922 seconds. Current values are obtained with close agreement with the SIMULINK battery model shown in Figure 6.29. 


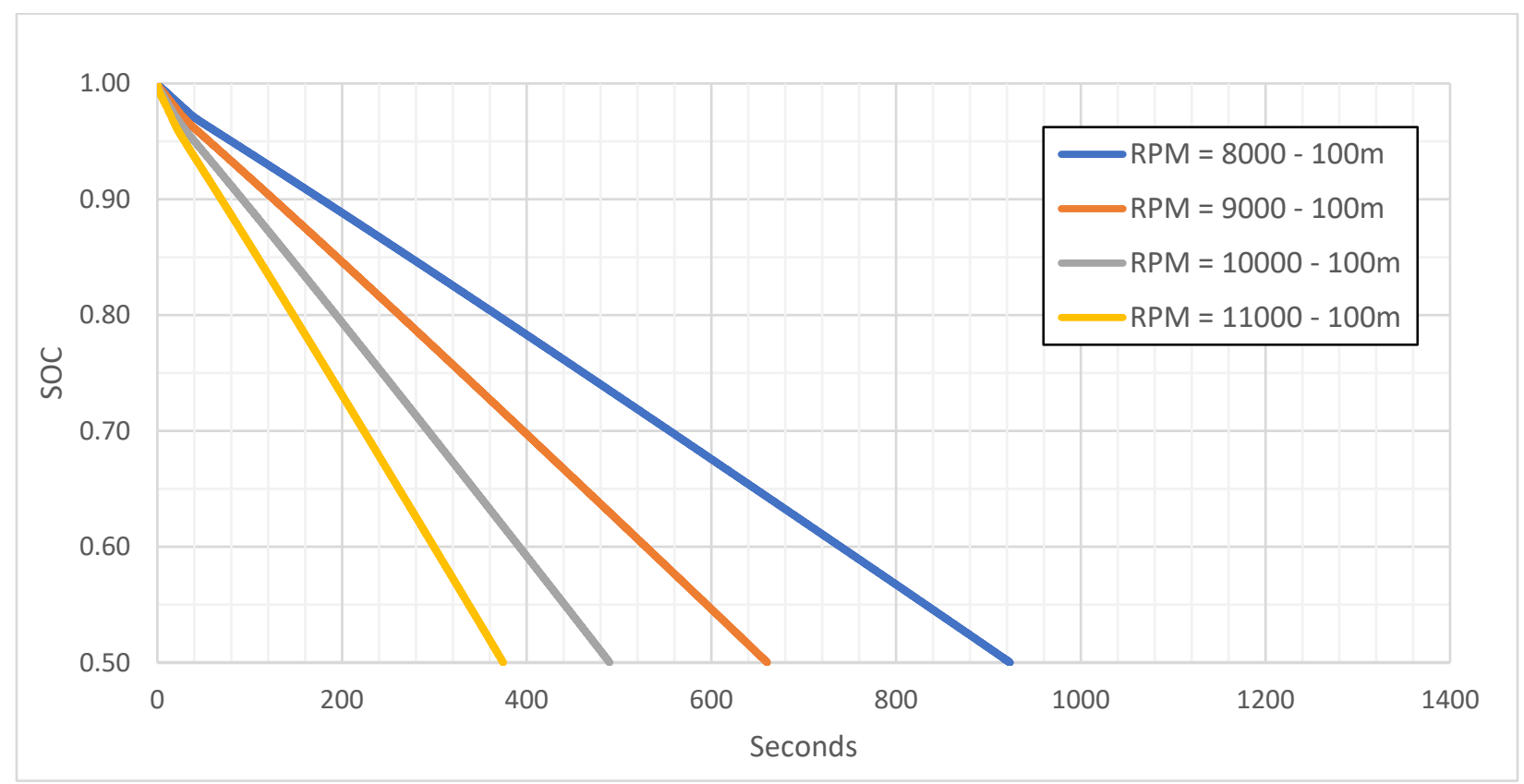

Figure 6.28 SOC vs Time plot with ranging RPM values

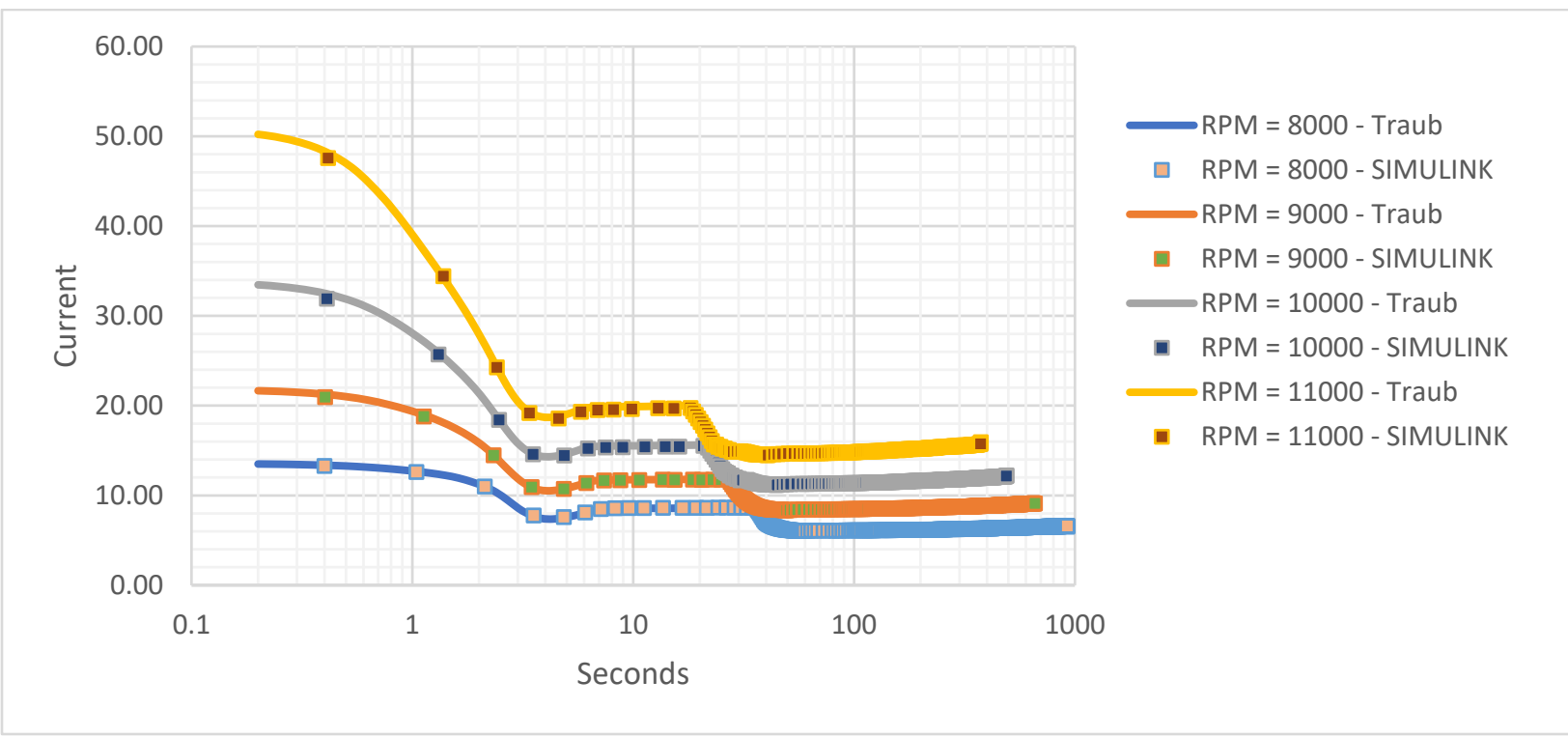

Figure 6.29 Current vs Time plot for ranging RPM values with SIMULINK simulation points 


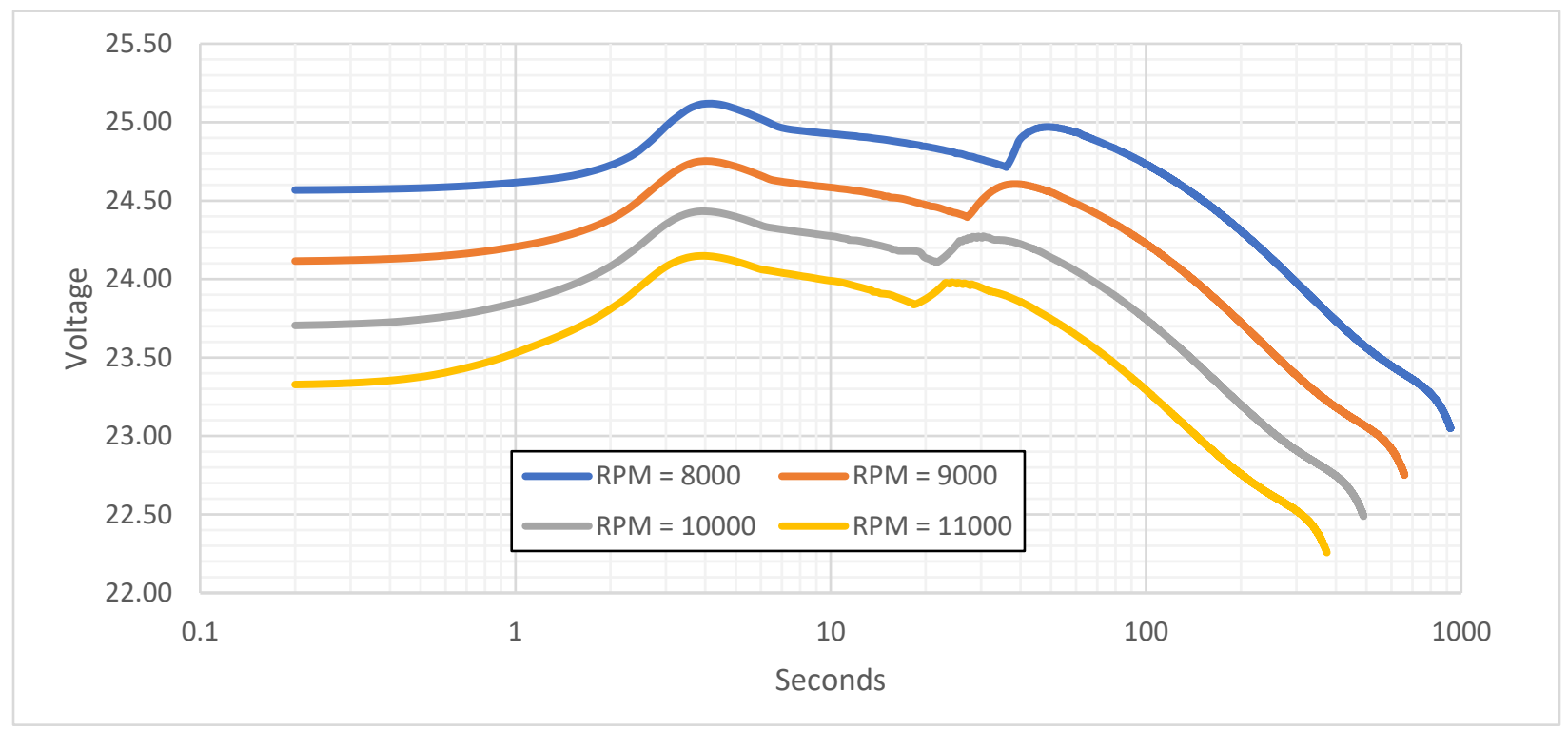

Figure 6.30 Voltage drop plot over simulation time for ranging RPM values

This time, voltages are compared with the SIMULINK battery model solutions. Some RPM values have yielded a better fit. However, all validation points gave an acceptable agreement. The trends in the voltage drops seem to differ the most in the post-transient discharge regions. This is where the constant current discharge battery method struggles the most to match. 


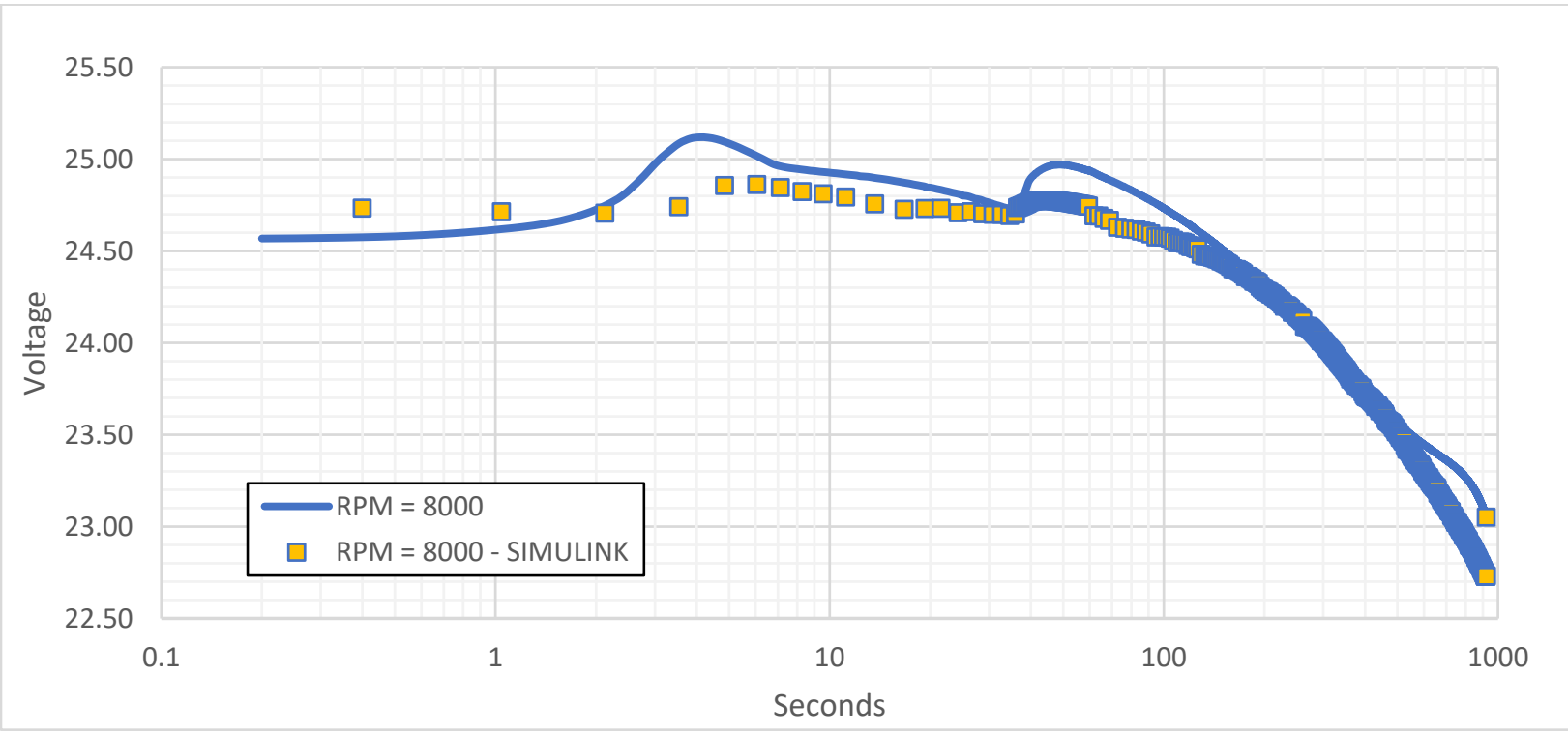

Figure 6.31 Voltage drop plot for RPM = 8000 compared with the SIMULINK values

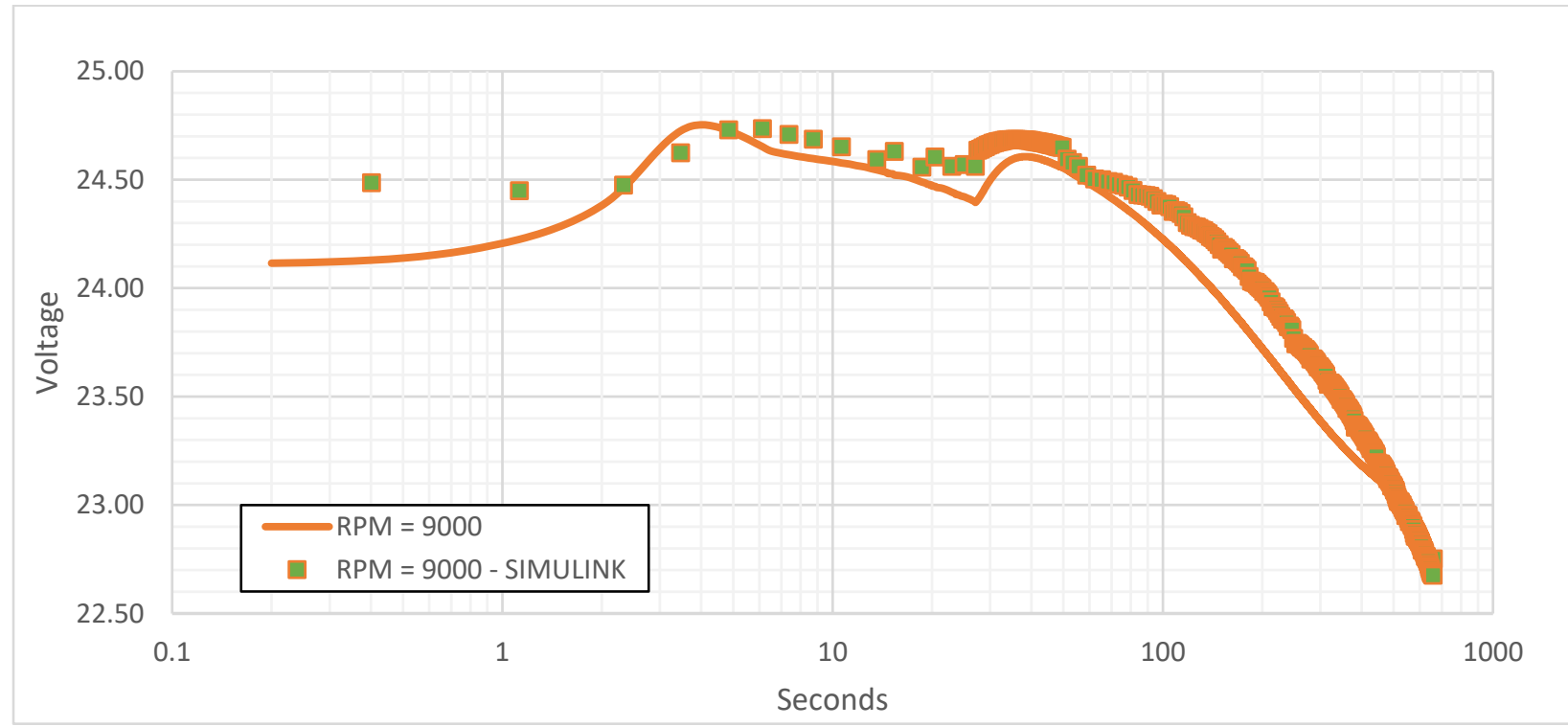

Figure 6.32 Voltage drop plot for RPM = 9000 compared with the SIMULINK values 


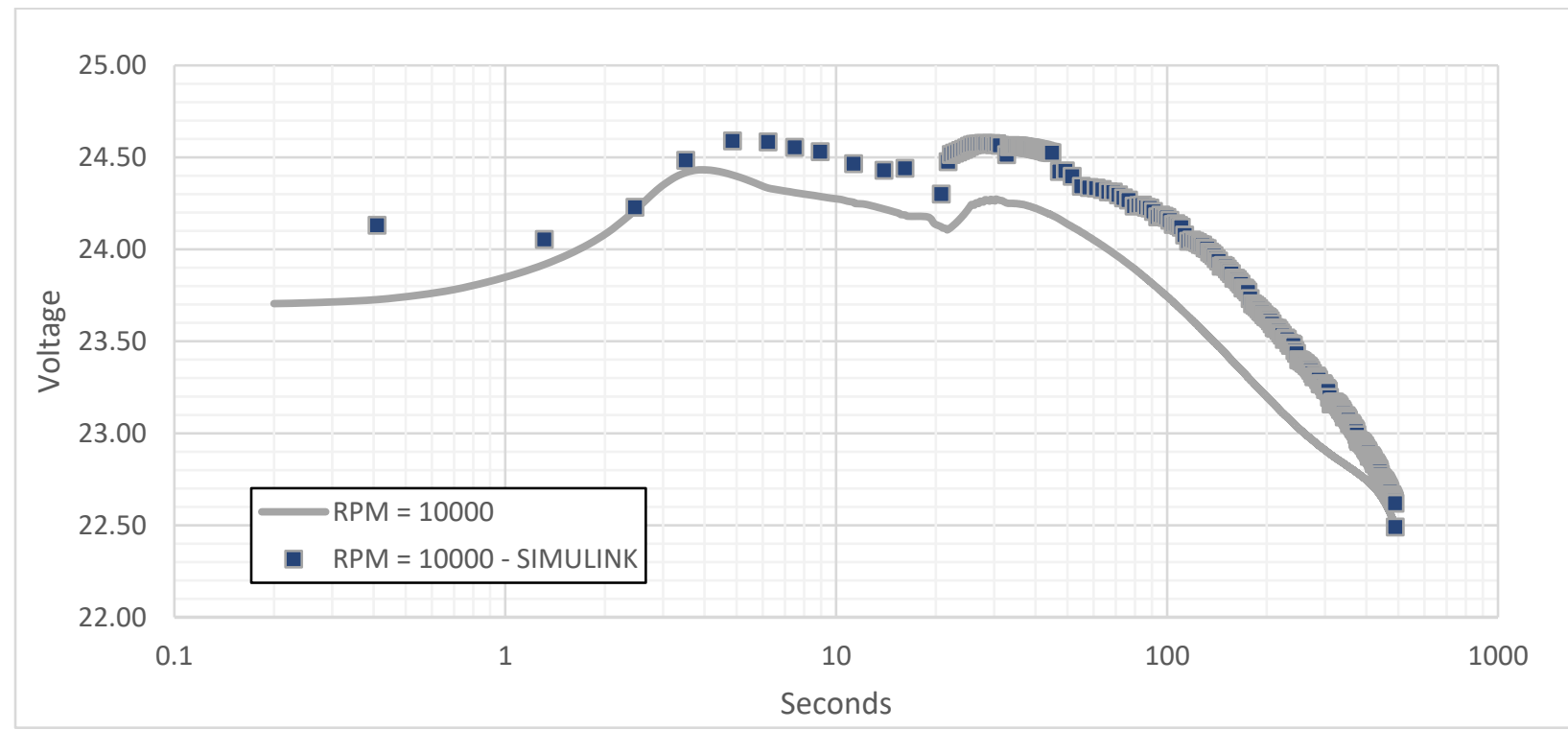

Figure 6.33 Voltage drop plot for RPM = 10000 compared with the SIMULINK values

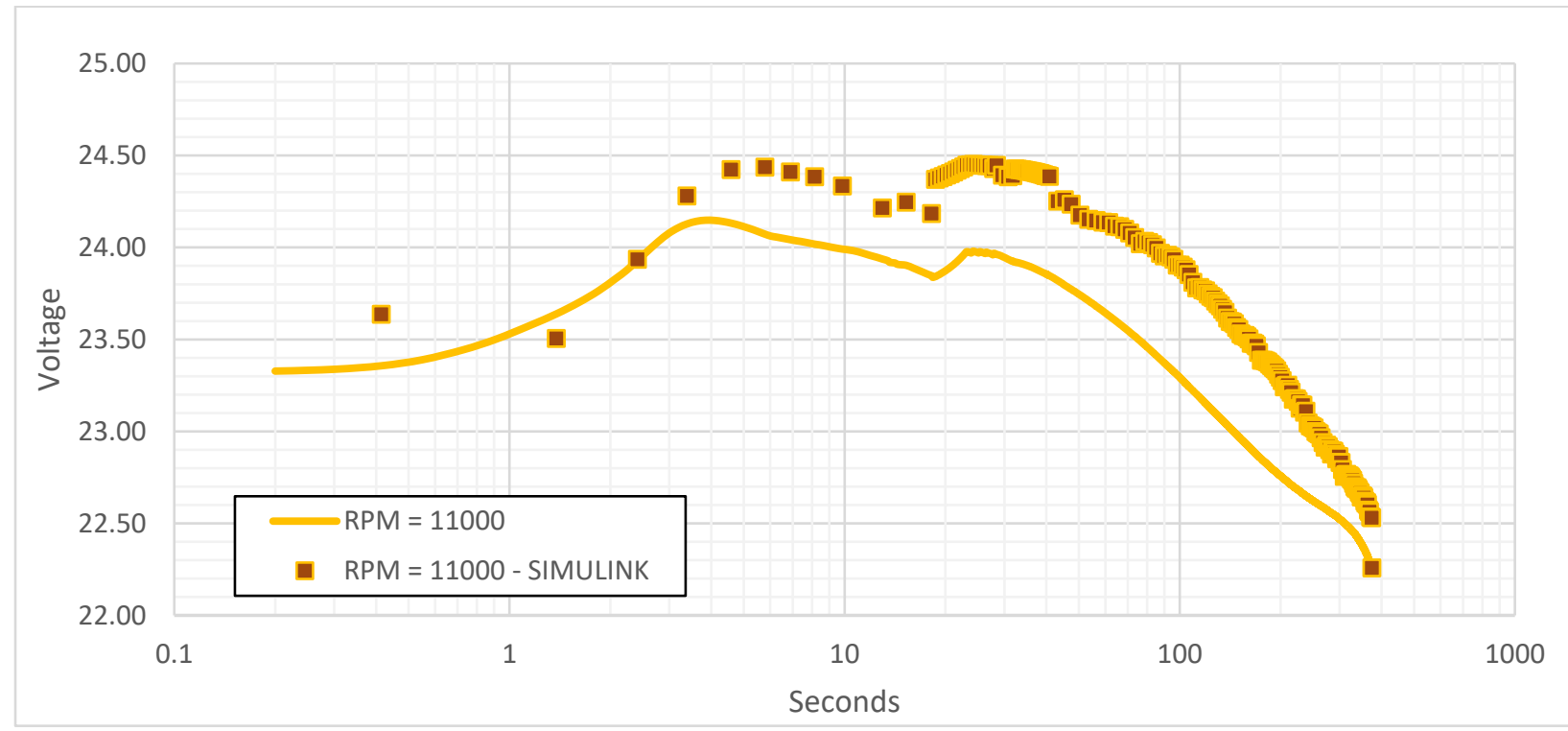

Figure 6.34 Voltage drop plot for RPM = 11000 compared with the SIMULINK values 
Lastly, computation times for the simulations are collected from MATLAB. The comparison plot has yielded the expected results like the previous mission shown in Figure 6.35.

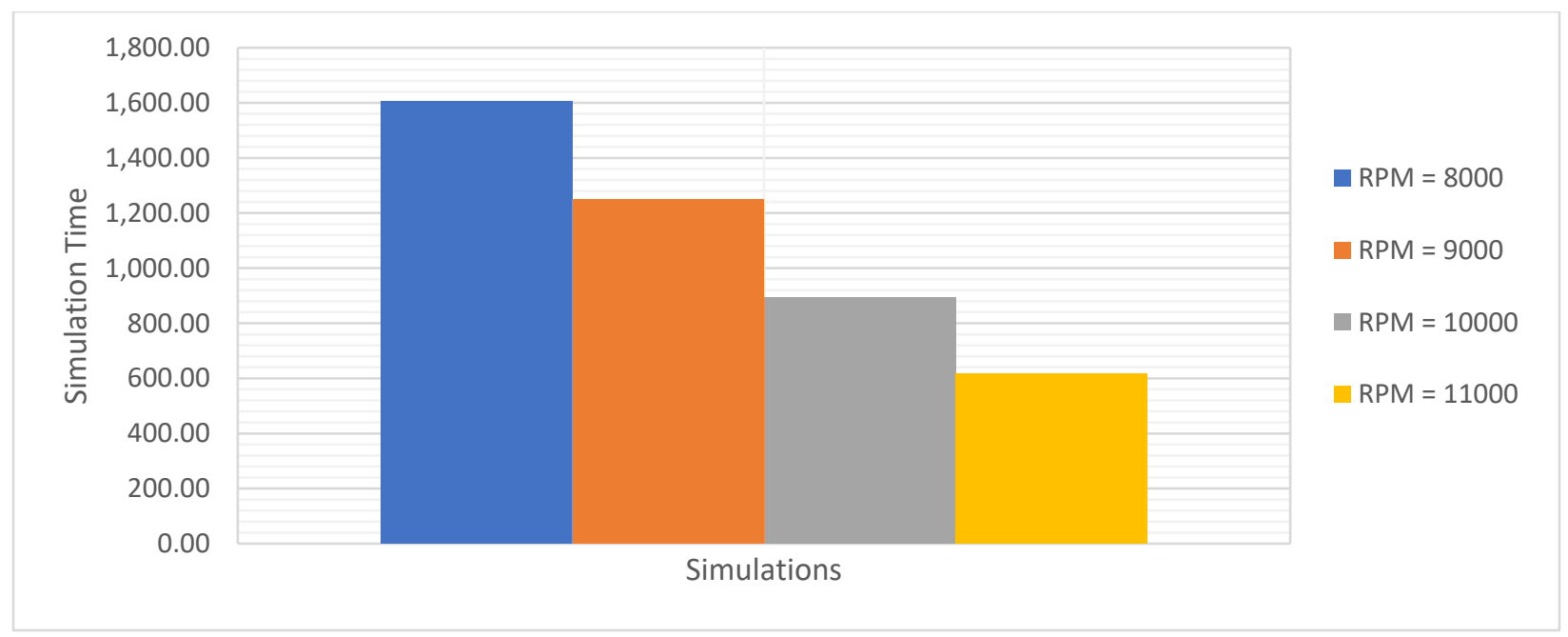

Figure 6.35 Computation time all simulations

\subsection{DISCUSSION}

Simulations were successfully implemented and performed in the MATLAB environment. The straight flight mission results indicate that higher range and endurance are experience at the lower RPM flight missions although the overall efficiencies of the drivetrain are shown to be slightly higher as the RPM goes up. This is due to the higher thrust generation and causes the higher current draw from the battery resulting in a faster battery drain. It can be seen that each 
1000RPM increase in the motor speed, thrust also increases by 1 to 2 Newtons at the stable flight region.

Changing the cruising level does not seem to affect the current levels of the different flight segments, but it delays the climb phase resulting in a decrease in range in this case. This decrease in range is more apparent in lower RPM settings.

Endurance values seem to be affected by the cruising altitude change, although not greatly; however, the higher cruising altitude results in a slightly lower endurance value. Approximately a 17 second difference is seen between $100 \mathrm{~m}$ and $200 \mathrm{~m}$ for $8000 \mathrm{RPM}$. This is dropped to approximately 6 seconds at 11000RPM. Hence, the higher RPM values are more efficient for the climb.

Similar to the straight flight mission, in turning flight, a lower RPM value of 8000 has both the highest of endurance and range values. Another thing to point out in this mission is that although the heading command subroutine has given a higher bank angle due to the higher sufficient speed possible at 11000RPM, the tighter turn has been achieved by the lower RPM value due to the lower speed resulting in a tighter turn radius. Also, a steeper climb is achieved by the higher RPM value as in the first mission.

The battery model is also validated in this mission. The current values seem to be in very close agreement with the developed SIMULINK model for the same battery. However, the same accuracy is not obtained in the voltage values, but this is expected since the experiments conducted for the constant current method only estimate the constant discharge accurately and interpolate the in between values, unlike a pulse discharge performed for the SIMULINK model in which both voltages drop, and recovery regions are analyzed and modelled. 
In both missions, the simulation that has both the higher endurance and range is also the most computationally expensive. This is also expected since more calculations are required with these simulations.

\subsection{Validation}

Although the validation for the whole simulation including the 3-D trajectory with full flight attitudes and speed is not achieved through this thesis, there are a couple of checks we can do to make sure simulation meets sensible operation criteria. These sanity checks are done to the simulation code for take-off and climb phases are shown below.

I. Take-off Validation

\begin{tabular}{|c|c|c|c|c|c|c|c|c|c|c|c|}
\hline \multicolumn{9}{|l|}{ 8000Rpm } & \multicolumn{9}{l|}{ 9000Rpm } & \multicolumn{1}{l|}{ 10000Rpm } & \multicolumn{3}{l|}{ 11000Rpm } \\
\hline$\underline{\text { Time }}$ & $\underline{\text { Distance }}$ & $\underline{\text { Velocity }}$ & $\underline{\text { Time }}$ & $\underline{\text { Distance }}$ & $\underline{\text { Velocity }}$ & $\underline{\text { Time }}$ & $\underline{\text { Distance }}$ & $\underline{\text { Velocity }}$ & $\underline{\text { Time }}$ & $\underline{\text { Distance }}$ & $\underline{\text { Velocity }}$ \\
\hline 0 & 0 & 0.01 & 0 & 0 & 0.01 & 0.0000 & 0.0000 & 0.0100 & 0.0000 & 0.0000 & 0.0100 \\
\hline 0.1 & 0.0095 & 0.0947 & 0.1 & 0.0118 & 0.1175 & 0.1000 & 0.0144 & 0.1442 & 0.1000 & 0.0174 & 0.1745 \\
\hline 0.2 & 0.0323 & 0.2282 & 0.2 & 0.0407 & 0.2897 & 0.2000 & 0.0506 & 0.3614 & 0.2000 & 0.0617 & 0.4425 \\
\hline 0.3 & 0.0736 & 0.4129 & 0.3 & 0.0936 & 0.5288 & 0.3087 & 0.1269 & 0.7022 & 0.3267 & 0.1846 & 0.9705 \\
\hline 0.4 & 0.1384 & 0.6486 & 0.4021 & 0.1797 & 0.8432 & 0.4178 & 0.2518 & 1.1450 & 0.4588 & 0.4102 & 1.7070 \\
\hline 0.5 & 0.2319 & 0.9350 & 0.5154 & 0.3248 & 1.2811 & 0.5561 & 0.5126 & 1.8846 & 0.5993 & 0.7890 & 2.6963 \\
\hline 0.6025 & 0.3634 & 1.2818 & 0.6430 & 0.5655 & 1.8864 & 0.7143 & 0.9783 & 2.9448 & 0.7728 & 1.5253 & 4.2431 \\
\hline 0.7221 & 0.5742 & 1.7626 & 0.7980 & 0.9977 & 2.7879 & 0.9080 & 1.8617 & 4.5593 & 0.9758 & 2.8348 & 6.4513 \\
\hline 0.8564 & 0.8957 & 2.3939 & 0.9757 & 1.7123 & 4.0216 & 1.1262 & 3.3277 & 6.7211 & 1.2021 & 4.9460 & 9.3287 \\
\hline 1.0003 & 1.3514 & 3.1674 & 1.1655 & 2.7634 & 5.5388 & 1.3545 & 5.4463 & 9.2778 & 1.4339 & 7.8578 & 12.5646 \\
\hline 1.1699 & 2.0666 & 4.2152 & 1.3876 & 4.4446 & 7.5688 & 1.5902 & 8.3038 & 12.1227 & 1.6643 & 11.5215 & 15.8965 \\
\hline 1.3648 & 3.1538 & 5.5780 & 1.6285 & 6.8489 & 9.9787 & 1.8325 & 11.9667 & 15.1208 & 1.8965 & 15.9799 & 19.2002 \\
\hline 1.5840 & 4.7482 & 7.2760 & 1.8779 & 9.9836 & 12.5728 & 2.0827 & 16.5057 & 18.1387 & & & \\
\hline 1.8205 & 6.9338 & 9.2393 & 2.1197 & 13.6217 & 15.0448 & & & & & & \\
\hline 2.0772 & 9.8704 & 11.4419 & 2.3714 & 18.0239 & 17.4879 & & & & & & \\
\hline 2.3401 & 13.4611 & 13.6556 & & & & & & & & & \\
\hline 2.6395 & 18.2643 & 16.0429 & & & & & & & & & \\
\hline
\end{tabular}

Table 6.2 Take-off run calculation parameter for ranging RPM values from the simulation 
In Table 6.2 simulation runs are displayed for the take-off portions of each RPM value. These depict a good solution for the take-off phase for the simulation. However, the better resolution can be achieved by setting a very small timestep for the take-off phase. This recalculation could yield a better solution than can be validated with the textbook formulas.

The more refined end results are shown in Table 6.3.

\begin{tabular}{|l|l|l|l|l|}
\hline & 8000Rpm & 9000Rpm & 10000Rpm & 11000Rpm \\
\hline Take-off Speed $(\mathrm{m} / \mathrm{s})=$ & 14.533 & 14.5099 & 14.5308 & 14.4810 \\
\hline Time $(\mathrm{sec})=$ & 2.67 & 2.27 & 1.98 & 1.75 \\
\hline TO distance $(\mathrm{m})=$ & 14.7197 & 12.1852 & 10.4834 & 9.1298 \\
\hline
\end{tabular}

Table 6.3 Refined final parameters for Take-off

The textbook solution for the take-off speed calculated from Eq. (6.1) is

$$
V_{S 1}=\sqrt{\frac{2 W}{\rho S C L_{\max }}}
$$

This is defined by the design textbook as stalling speed. The condition for take-off sets to the stalling speed the simulation. If we replace the maximum lift coefficient with the take-off lift coefficient calculated at the simulation, the typical value obtained is shown in Eq. (6.2).

$$
V_{S 1}=14.51 \mathrm{~m} / \mathrm{s}
$$


This shows a very close agreement with the simulation results. The variation of the take-off speed in our simulation is mainly caused by the different thrust values in each simulation causing the slight change in lift because of the presence of the 1 deg angle of attack created by the nominal pitch angle of the aircraft on the ground.

The final validation check id done with the real RC model flown in the field. The flight path flown includes a series of loiters on a field due to the field restriction. This creates a noisy flight profile for the real-life data. However, we can extract the speed and motor RPM value to perform a check with the simulation.

Figure 6.36 and Figure 6.37 show the flight data obtained by using the data acquisition module in the AVISTAR RC airplane.

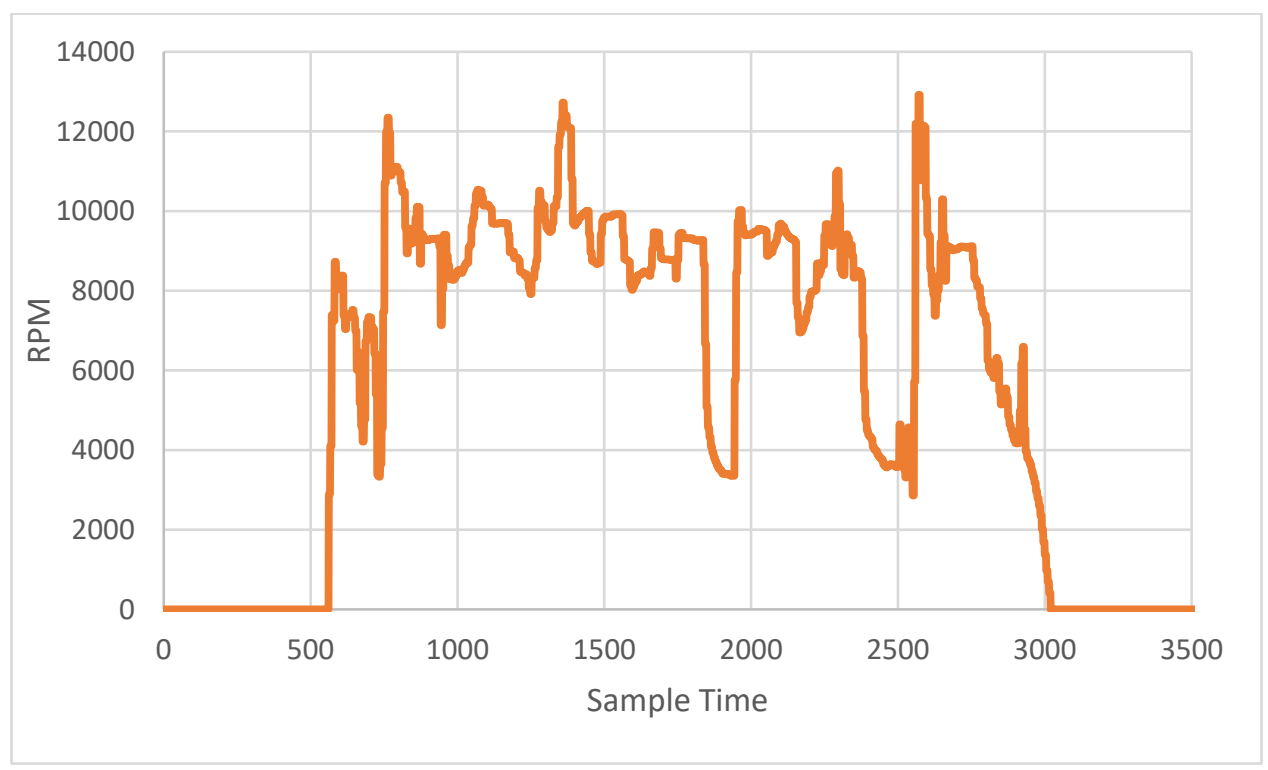

Figure 6.36 RPM vs the flight sample time from flight data 


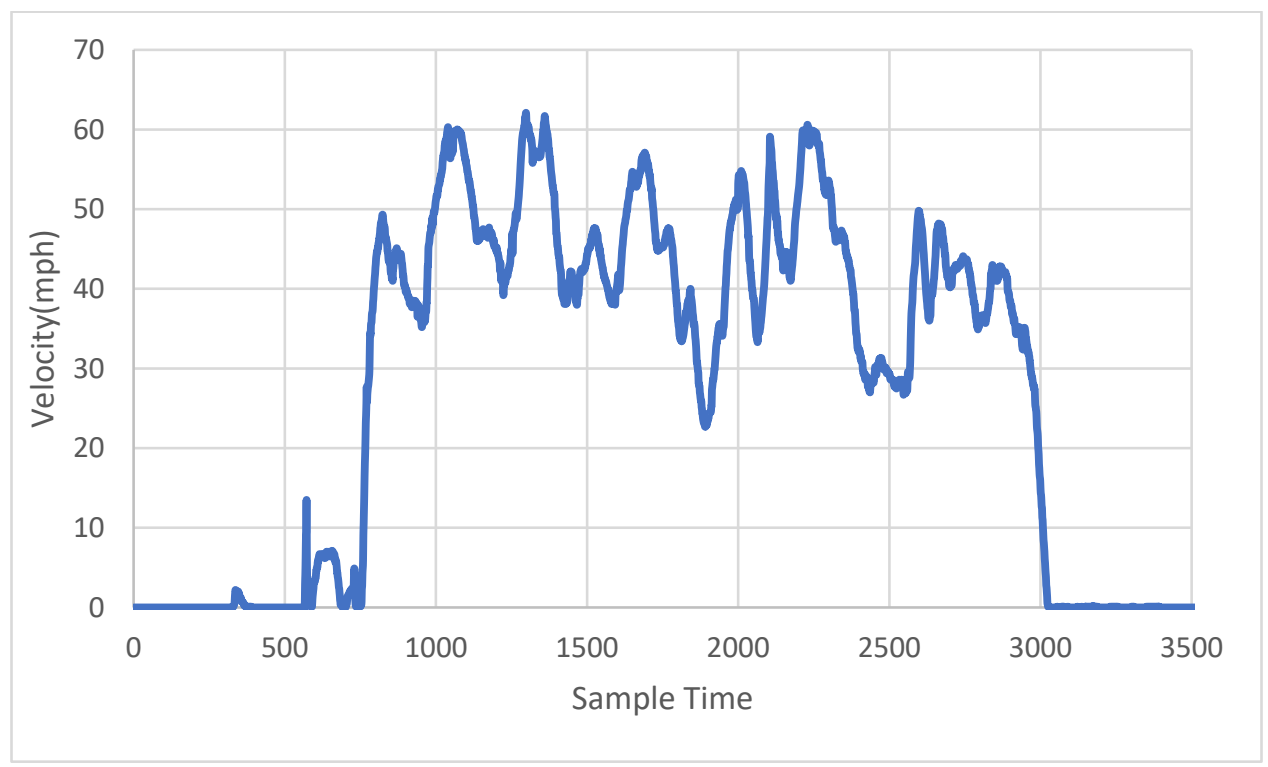

Figure 6.37 Velocity vs the flight sample time from flight data

The results from the simulation data show a similar value of the velocity. In Table 6.4 comparison is being made between the correlation of the velocity data on both the simulation and the flight data.

\begin{tabular}{|l|l|l|l|l|}
\hline & 8000Rpm & 9000Rpm & 10000Rpm & 11000Rpm \\
\hline Flight data Speed $(\mathrm{m} / \mathrm{s})=$ & 17.88 & 20.16 & 23.78 & 26.64 \\
\hline Simulation Speed $(\mathrm{m} / \mathrm{s})=$ & 18.47 & 21.72 & 25.06 & 28.47 \\
\hline
\end{tabular}

Table 6.4 flight data and simulation velocity comparison with various RPM levels 
The data shows that the simulation was fairly close to real-life values. However, environmental factors such as a wind speed and other weather conditions might affect the quality of the data obtained from the real-life flight. Also, the flight is controlled manually which has caused noisy data and made it harder to read the precise values from the data. 


\section{CHAPTER 7}

\section{CONCLUSION \& FUTURE WORK}

\subsection{Conclusion}

A prototype trajectory analysis simulator for electrically powered UAVs has been demonstrated and includes simulated lithium-polymer battery modeling throughout selected mission profiles. The battery model implemented initially is that inspired by Lance Traub [7]. This battery model is specially constructed to work more accurately with air vehicles. Most other conventional battery models are based on constant current discharge data. This contradicts the nature of the flying mission. The battery powered propeller powertrain uses a nearly constant power value when flying conditions are stable (cruise, constant rate turn). This poses an issue with the regular battery models which rely on the constant discharge data. In the flying case, power is kept constant at these stages, but the current has shown an upward trend through the simulation. The constant power discharge method is considered to be a fix for this issue of modelling battery on air vehicle simulations.

Sub-systems such as aerodynamics and propulsion modules were integrated. A combination of empirical methods as well as using external software called "XFLR5," which utilizes the VLM (Vortex-Lattice Method) for 3-D panel shaped surfaces, are use to obtain the aerodynamics of the aircraft. Lift and drag polars are obtained with these methods, and look-up tables are used for the simulation.

Experimental analyses were performed in the ODU UAV lab for the constant battery model that was described previously, and the SIMULINK Simscape ${ }^{\mathrm{TM}}$ battery model was constructed for 
the validation. For the primary method, three separate experiments were carried out. These three experiments involved the constant current discharge for 10A, 20A and 30A discharge rates covering the operation envelope of the battery during flight. They were crude and have demonstrated the method but could benefit from improved experimental techniques. A pulse discharge simulated experiment was carried out for the SIMULINK battery model at the same conditions to provide a fair test bed.

Two different missions were considered in the concept of this thesis. One of which is the take-off, climb to cruise altitude and fly until the battery SOC level drains to the selected level. The other mission is very similar to the first mission but this time incorporating the turning command into the simulation which makes this the three-dimensional case for our investigation.

Trajectories for a range of different RPM values were analyzed. The simulation computing time was also collected to see the difference in computational load of each simulation. Lastly, a validation check has been made for the battery model, and the results show promising agreement of the SOC and current draw values between the developed method and the SIMULINK method.

\subsection{Future Work}

One of the more essential parts of this thesis is the aircraft dynamics model and the battery model since the main focus behind this investigation of the trajectory simulation is to understand and estimate the battery performance of a UAV type aircraft that is powered by a lithium-polymer battery. Through the methodology of the thesis there are some parts which are established with taking compromises on the implementation of the methods. There is also a need for a formal validation. To summarize, future actions could include: 
1. Aircraft dynamics model: Aircraft dynamics model used in this simulation objective can be improved to the 6-DOF freedom with proper control and stability equations using full flight mechanics method which computes forces and moments on a 3-D body. Achieving a fully parametric dynamics model could potentially help to get more accurate mission paths and enable generation of more complex trajectories.

2. Battery Model: The battery model selected in this thesis is a combination of experimental data and clever use of available data to manipulate the solution fit to the needs of the simulation. This still can be improved with the more involved model methods which can give a better approximation to the SOC and voltage values overall for the mission.

3. The drive motor and drive train modeling could be improved to better represent changes in power available. Higher order motor efficiency formulation can be adopted for the better representation of the drivetrain power and energy calculations.

4. Finally, a real-life flight test can be fully incorporated into the project validation to compare the results in a more tangible and detailed manner. With the use of an autopilot and flight data recorder, trajectories can be commanded to the AVISTAR UAV which is currently in development at ODU. A similar autopilot algorithm can be generated for the new simulation, and the correlations can be drawn from the real-life flight test to the simulation the UAV. 


\section{REFERENCES}

[1] L. W. Traub, "Range and Endurance Estimates for Battery-Powered Aircraft," Journal of Aircraft Vol. 48 No. 2, , March-April 2011.

[2] T. Donateo, A. Ficarella, L. Spedicato, A. Arista and M. Ferraro, "A new approach to calculating endurance in electric flight and comparing fuel cells and batteries," Applied Energy, vol. 187, pp. 807-819, 2017.

[3] N. Lapena-Rey, J. Blanco, E. Ferreyra, J. Lemus, S. Pereira and E. Serrot, "A fuel cell powered unmanned aerial vehicle for low altitude surveillance missions," International journal of Hyrdrogen Energy, vol. 42, no. 10, pp. 6926-6940, 2017.

[4] M.-h. Hwang, H.-R. Cha and S. Y. Jung, "Practical Endurance Estimation for Minimizing Energy Consumption of Multirotor Unmanned Aerial Vehicles," Energies, vol. 11, p. 2221, 2018.

[5] F. Cappello, S. Bijjahalli, S. Ramasamy and R. \& Sabatini, "Aircraft Dynamics Model Augmentation for RPAS Navigation and Guidance," in International Conference on Unmanned Aircraft Systems (ICUAS), Arlington, VA, 2016.

[6] O. D. Dantsker, M. Vahora, S. Imtiaz and C. Marco, "High Fidelity Moment of Inertia Testing of Unmanned Aircraft," in Applied Aerodynamics Conference, Atlanta, Georgia, 2018. 
[7] W. L. Traub, "Calculation of Constant Power Lithium Battery Discharge Curves," Batteries, 2016, 2, 17.

[8] B. Bole, M. Daigle and G. Gorospe, "Online Prediction of Battery Discharge and Estimation of Parasitic Loads for an Electric Aircraft," NASA Ames Research Center, Moffett Field, CA, 2014.

[9] D. Linden and T. B. Reddy, "Chapter 1 Basic Concepts," in Handbook of Batteries 3rd edition, New York, NY, McGraw-Hill, 2002, pp. 1.1-1.6.

[10] A. Miele, "Chapter 4:Scalar Equations for Flight Over a Flat Earth," in Flight Mechanics: Theory of Flight Paths, Reading,Massachusetts, Dover Publications, 2016, p. 51.

[11] A. Miele, "Chapter 4:Scalar Equations for Flight Over a Flat Earth," in Flight Mechanics: Theory of Flight Paths, Reading,Massachusetts, Dover Publications, 2016, pp. 42-50.

[12] D. G. Hull, "3DOF Equations of Motion," in Fundamentals of Airplane Flight Mechanics, New york, Springer, 2007, pp. 28-34.

[13] T. Huria, M. Ceraolo, J. Gazzarri and R. Jackey, "High fidelity electrical model with thermal dependence for characterization and simulation of high power lithium battery cells," in IEEE International Electric Vehicle Conference, Greenville, SC, 2012.

[14] I. Baccouche, S. Jemmali, A. Mlayah, B. Manai and N. E. B. Amara, "Implementation of an improved Coulomb-Counting Algorithm Based on a Piecewise SOC-OCV Relationship for SOC Estimation of Li-Ion Battery," ArXiv, vol. abs/1803.10654, 2018. 
[15] N. Kong, M. Chin-Sien, C. Yi-Ping and H. Yao-Ching, "Enhanced Coulomb counting method for estimating state-of-charge and state-of-health of lithium-ion batteries," Applied Energy, vol. 86, no. 9, pp. 1506-1511, 2009.

[16] R. Xiong, HongwenHE and K. Zhao, "Research on an Online Identification Algorithm for a Thevenin," International Journal of Green Energy, vol. 12, pp. 272-278, 2015.

[17] M. Murnane and A. Ghazel, "A Closer Look at State of Charge (SOC) and State of Health (SOH) Estimation Techniques for Batteries," 2018.

[18] R. Zhang, B. Xia, B. Li, L. Cao, Y. Lai, W. Zheng, H. Wang and W. Wang, "State of the Art of Lithium-Ion Battery SOC Estimation for Electrical Vehicles," Energies , vol. 11, p. 1820, 2018.

[19] M. A. Roscher, J. Assfalg and O. S. Bohlen, "Detection of Utilizable Capacity Deterioration in Battery Systems," IEEE Transactions on Vehicular Technology, vol. 60, no. 1, pp. 98$103,2011$.

[20] S. Lee, J. Kim, J. Lee and B. H. Cho, "State-of-charge and capacity estimation of lithiumion battery using a new open-circuit voltage versus state-of-charge," J. Power Sources, vol. 185, no. 2, pp. 1367-1373, 2008.

[21] J. P. Christopherson, Battery Test Manual For Electric Vehicles, Idaho Falls, Idaho: The Idaho National Laboratory is a U.S. Department of Energy National Laboratory, 2015, pp. $6-9$.

[22] D. Rowell, "2.161 Signal Processing: Continuous and Discrete," Massachusetts Institute of Technology: MIT OpenCourseWare, Fall 2018. 
[23] G. M. Siouris, "The Generalized Missile Equations of Motion," in Missile Guidance and Control Systems, Springer, 2004, pp. 36-37.

[24] A. Miele, "Chapter 4:Scalar Equations for Flight Over a Flat Earth," in Flight Mechanics: Theory of Flight Paths, Reading,Massachusetts, Dover Publications, 2016, p. 49.

[25] S. Gudmundsson, "Performance Take-off," in General Aviation Aircraft Design: Applied Methods and Procedures, Elsevier, 2014, p. 799.

[26] S. Gudmundsson, "Performance Take-off," in General Aviation Aircraft Design: Applied Methods and Procedures, Elsevier, 2014, p. 798.

[27] E. Fehlberg, Classical fifth-, sixth-, seventh-, and eighth-order runge-kutta formulas with stepsize control, Huntsville: NASA, October 1968.

[28] J. Dormand and P. Prince, "High order embedded Runge-Kutta formulae," Journal of Computational and Applied Mathematics, vol. 7, no. 1, pp. 67-75, 1981.

[29] J. How, "16.333 Aircraft Stability and Control," Massachusetts Institute of Technology: MIT OpenCourseWare, Fall 2004.

[30] "Great Planes Rimfire .46 42-60-800 Outrunner Brushless," Tower Hobbies, [Online]. Available: https://www.towerhobbies.com/cgi-bin/wti0001p?I=GPMG4725. [Accessed 13 October 2019].

[31] M. Drela, "Course notes on simple electric motor model and motor/prop matching," 3 March 2005. [Online]. Available: http://web.mit.edu/drela/Public/web/qprop/motorprop.pdf. [Accessed 1310 2019]. 
[32] B. W. McCormick, "Second Edition Aerodynamics, Aeronautics, and Flight Mechanics," John Wiley \& Sons, Inc, 1995, pp. 297-301.

[33] "APC Propeller Performance Data," APC Propellers, [Online]. Available: https://www.apcprop.com/technical-information/performance-data/. [Accessed 13 October 2019].

[34] J. B. Brandt, R. W. Deters, G. K. Ananda and M. S. Selig, "UIUC Propeller Data Site," UIUC, 11 November 2015. [Online]. Available: https://mselig.ae.illinois.edu/props/propDB.html. [Accessed 13 Octover 2019].

[35] "xflr5," July 2019. [Online]. Available: http://www.xflr5.tech/xflr5.htm. [Accessed 28 October 2019].

[36] L. Clark, "MIT Aero-Astro Magazine-Mark Drela Profile," MIT, [Online]. Available: http://web.mit.edu/aeroastro/news/magazine/aeroastro-no3/2006drela.html. [Accessed 28 October 2019].

[37] O. D. Dantsker, "Determining Aerodynamic Characteristics of an Unmanned Aerial Vehicle using a 3D Scanning Technique," in AIAA SciTech Forum, Kissimmee, Florida, 2015.

[38] S. Gudmundsson, General Aviation Aircraft Design: Applied Methods and Procedures, Waltham, MA: ElsevierGudmundsson, Snorri, 2014.

[39] D. P. Raymer, Aircraft design: A conceptual approach, Washington, D.C: American Institute of Aeronautics and Astronautics, 1989. 
[40] A. Deperrois, "Part IV - Theoretical limitations and shortcomings of xflr5," June 2019. [Online]. Available: http://xflr5.tech/docs/Part\%20IV:\%20Limitations.pdf. [Accessed 28 October 2019].

[41] S. Gudmundsson, "Chapter 15 - Aircraft Drag Analysis," in General Aviation Aircraft Design: Applied Methods and Procedures, Waltham, MA, Elsevier, 2014, pp. 680-681.

[42] R. S. Shevell, Fundamentals of Flight, Lebanon, Indiana: Prentice Hall, 1983, p. 179.

[43] S. Gudmundsson, "Chapter 15 - Aircraft Drag Analysis," in General Aviation Aircraft Design: Applied Methods and Procedures, Waltham, MA, Elsevier, 2014, pp. 700-703.

[44] Y.-H. Sun, H.-L. Jou and J.-C. Wu, "Multilevel Peukert equations based residual capacity estimation method for lead-acid battery," in IEEE International Conference on Sustainable Energy Technologies, Singapore, 2008.

[45] A. Hasan, M. Skriver and T. A. Johansen, "Exogenous Kalman Filter for State-of-Charge Estimation in Lithium-Ion Batteries," IEEE Conference on Control Technology and Applications (CCTA), pp. 1403-1408, 2018.

[46] T. Huria, M. Ceraolo, J. Gazzarri and R. Jackey, "High fidelity electrical model with thermal dependence for characterization and simulation of high power lithium battery cells," in IEEE International Electric Vehicle Conference, Greenville, SC, 2012.

[47] B. J. Brelje and J. R. Martins, "Development of a Conceptual Design Model for Aircraft Electric Propulsion with Efficient Gradients," in AIAA/IEEE Electric Aircraft Technologies Symposium, Cincinati,Ohio, 2018. 
[48] "Great Planes Avistar Elite. 46 Advanced Trainer RTF," Great Planes, 2019. [Online]. Available: http://www.greatplanes.com/airplanes/gpma1605.php. [Accessed 13 November 2019].

[49] "UIUC Airfoil Data Site," UIUC Applied Aerodynamics Group, 2019. [Online]. Available: https://m-selig.ae.illinois.edu/ads/coord_database.html. [Accessed 13 November 2019].

[50] "The brushless advantage for outrunner design motors!," Great Planes, [Online]. Available: http://www.greatplanes.com/motors/gpmg4505.php. [Accessed 11 November 2019].

[51] "12x6E|APC Propellers," APC Propellers, [Online]. Available: https://www.apcprop.com/product/12x6e/. [Accessed 11 November 2019].

[52] R. A. Bunge, "Aircraft Flight Dynamics," AA241X, Stanford University, April 132015.

[53] P. H. Zipfel, "Overview,Kinematics of Translation and Rotation," in Modelling and Simulation of Aerospace Vehicle Dynamics, Reston,VA, AIAA, 2007, pp. 1-4,126. 
VITA

NAME: $\quad$ Ege Konuk

DATA OF BIRTH: June $25^{\text {th }}, 1995$

\section{PERSONAL STATEMENT}

Highly passionate and detail oriented Aeronautical Engineer who has completed his bachelor's degree and now pursuing his Master of Science degree in Aerospace Engineering. Engaged and open to learn in every chance to develop experience and knowledge in engineering. Ambitious to work with accomplished and experienced individuals on his future studies.

\section{EDUCATION}

○ Old Dominion University

MSc Aerospace Engineering 2017-Present

- University of Turkish Aeronautical Association (UTAA)

BSc Aeronautical Engineering Class of 2017

WORK EXPERIENCE

\section{Reliability and Safety Intern}

TUSAS Aerospace Industries, Inc. (TAI)

Jun. 2015 - Jul. 2015

- The Job was to file the inputs into fault tree with using Software's that interpret those inputs and help engineers for documentation of each part for the current Project.

\section{Academic Intern}

University of Turkish Aeronautical Association

Jun. 2016 - Jul. 2016

\section{KEY SKILLS.}

\section{Computer Skills}

I. Programming Languages

- MATLAB/SIMULINK(Advanced), FORTRAN(Intermediate), PYHTON(Novice)

II. CFD Software

- ANSYS FLUENT, SCFLOW

III. CAD Software

- SOLIDWORKS

IV. Other Software

- MS Office, TECPLOT 